\title{
IRREGULAR HODGE FILTRATION OF SOME CONFLUENT HYPERGEOMETRIC SYSTEMS
}

\author{
ALBERTO CASTAÑO DOMÍNGUEZ AND CHRISTIAN SEVENHECK
}

\begin{abstract}
We determine the irregular Hodge filtration, as introduced by Sabbah, for the purely irregular hypergeometric $\mathcal{D}$-modules. We obtain in particular a formula for the irregular Hodge numbers of these systems. We use the reduction of hypergeometric systems from GKZ-systems as well as comparison results to Gauß-Manin systems of Laurent polynomials via Fourier-Laplace and Radon transformations.
\end{abstract}

\section{INTRODUCTION}

The aim of this paper is to compute some Hodge theoretic invariants of certain classical differential systems in one variable. These are the so-called irregular Hodge numbers, which have been introduced recently by Sabbah Sab18a. They are called irregular because they are attached to differential systems which may have irregular singular points, a feature that is excluded for classical variations of Hodge structures as well as for the more general Hodge modules. The very definition of these numbers rely on the theory of mixed twistor $\mathcal{D}$-modules of T. Mochizuki (|Moc15a $]$ ). Twistor $\mathcal{D}$-modules generalise Hodge modules, in the sense that the underlying $\mathcal{D}$-module of a twistor $\mathcal{D}$-module can have irregular singularities. In particular, one can define a version of the Fourier-Laplace transformation functor within the category of mixed twistor $\mathcal{D}$-modules, which is impossible for mixed Hodge modules. The drawback of this generalization is that one cannot directly assign a filtration to a twistor $\mathcal{D}$-module, and hence it is not easy to attach numerical invariants like Hodge numbers to it. In the above mentioned paper Sab18a], Sabbah constructs an intermediate category between mixed Hodge modules and mixed twistor $\mathcal{D}$-modules (called irregular mixed Hodge modules) which is on the one hand sufficiently large to be stable under all relevant operations that are defined for twistor $\mathcal{D}$-modules (in particular, the Fourier-Laplace transformation), but which allows one to define a filtration, called irregular Hodge filtration, for each of its objects. The construction is related to the earlier papers [Yu14, ESY17, SY15], where for certain projective morphisms $f: X \rightarrow \mathbb{P}^{1}$, a (rationally indexed) filtration was introduced on the twisted de Rham complex $\left(\Omega^{\bullet}(* D), d+d f \wedge\right)$, where $D \subset X$ is a certain normal crossing boundary divisor such that $f_{\mid X \backslash D}$ yields a regular function. Instead of considering meromorphic differential forms, one can also use the subcomplex of so-called $f$-adapted logarithmic forms (also called Kontsevich complex, see [KKP17]) $\Omega_{f}^{\bullet}$, which consists of forms such that the exterior product with $d f$ is still logarithmic along $D$. From an $E_{1}$-degeneration property of the corresponding spectral sequence proved in [ESY17], one obtains a filtration on the twisted de Rham cohomology, called irregular Hodge filtration of the regular function $f: X \backslash D \rightarrow \mathbb{A}^{1}$. We refer to ESY17 for more details.

Notice also that the paper KKP17 gives three definitions of so-called Landau-Ginzburg Hodge numbers associated to a family $f: X \rightarrow \mathbb{P}^{1}$, one of them being $\operatorname{dim} H^{p}\left(\Omega_{f}^{q}\right)$. Conjecturally these three definitions coincide, but this seems to require some more assumptions (see [Sha18] and [Sab18b] for some partial results). Although these Hodge numbers have only integer indices, they are closely related to the dimensions of the graded parts of the filtration from [ESY17]. Ultimately, according to [KKP17] and following predictions from homological mirror symmetry, one hopes for a correspondence between the Hodge numbers of some, say, projective varieties entering in the A-model and the irregular Hodge numbers of its (Landau-Ginzburg) B-model.

2010 Mathematics Subject Classification. Primary 14F10, 32C38.

Key words and phrases. $\mathcal{D}$-modules, irregular Hodge filtration, hypergeometric systems, twistor $\mathcal{D}$-modules.

The authors are partially supported by the project SISYPH: ANR-13-IS01-0001-01/02 and DFG grant SE 1114/5-1. 
For the moment, there are quite a few examples where the irregular Hodge filtration can actually be computed. A central result of [Sab18a] states that rigid irreducible $\mathcal{D}$-modules on the projective line underlie objects of the category of irregular mixed Hodge modules, and consequently admit a unique irregular Hodge filtration, provided that their formal local monodromies are unitary. Rigid $\mathcal{D}$ modules are particularly interesting since they can be algorithmically constructed from simple objects by an algorithm due to Arinkin and Katz (cf. Ari10]). Among the most studied and best understood examples of such rigid $\mathcal{D}$-modules are the classical hypergeometric $\mathcal{D}$-modules. In the regular case (which corresponds to classical variations of complex Hodge structures), Fedorov has given in Fed18 a closed formula for the Hodge numbers (without computing the Hodge filtration itself however) conjectured by Corti and Golyshev in CG11] using the work [DS13] of Dettweiler and Sabbah.

In the present paper, we consider the case of purely irregular hypergeometric modules. Our principal result, Theorem 4.7, completely determines the irregular Hodge filtration and gives a very simple formula for the irregular Hodge numbers, which is in some sense similar to the shape of Fedorov's formula. The main ingredients are a reduction process (as explained in BMW18]) to obtain classical hypergeometric $\mathcal{D}$-modules from some higher dimensional ones, the so-called GKZ-systems, techniques from [Rei14] and RS15b, RS17] (going back to [Sab08]) to understand Hodge module structures on these GKZ-systems as well as a quite explicit solution to the so-called Birkhoff problem that is inspired by calculations in toric mirror symmetry (see again RS15b as well as [DS04 and also [GMS09]).

Since the first version of this paper was written, Hodge invariants for hypergeometric systems have been considered in some other articles. First, the formula for the Hodge numbers of purely irregular systems has also been obtained by Sabbah and Yu in the final version of Sab18a by different means. Another approach to Fedorov's formula for the case of regular systems (giving more precise information on the various Hodge invariants) has been given by N. Martin (see [Mar18]). Moreover, we have considered the case of hypergeometric operators of type $(n, 1)$ (see Definition 2.1 below) in a common paper with Th. Reichelt (CDRS18) using the computation of Hodge filtrations on GKZ-systems from RS15a. Finally, Sabbah and Yu have recently given a complete formula for the irregular Hodge numbers for all confluent hypergeometric systems in SY18. However, for the moment the irregular Hodge filtration itself is not determined in the general case.

Let us recall some notation that will be adopted throughout the paper. For a smooth complex algebraic variety $X$, we write $\mathcal{D}_{X}$ for the sheaf of algebraic differential operators on $X$. If $X$ is affine, we sometimes switch freely between sheaves of $\mathcal{D}_{X}$-modules and modules of global sections. We will denote the abelian categories of holonomic resp. regular holonomic $\mathcal{D}_{X}$-modules by $\operatorname{Mod}_{\mathrm{h}}\left(\mathcal{D}_{X}\right)$ resp. $\operatorname{Mod}_{\mathrm{rh}}\left(\mathcal{D}_{X}\right)$, and analogously with the respective bounded derived categories. For a morphism $f: X \rightarrow Y$, we denote the direct resp. inverse image functors for $\mathcal{D}$-modules as usual by $f_{+}$resp. $f^{+}$ (see HTT08 for a thorough discussion of these and related functors). We put $\mathbb{G}_{m}:=\operatorname{Spec} \mathbb{C}\left[t, t^{-1}\right]$; if we want to fix a coordinate on this one-dimensional torus, we also write $\mathbb{G}_{m, t}$.

We denote by $\mathcal{R}_{\mathbb{A}_{z}^{1} \times X}^{\text {int }}$ the sheaf of Rees rings on $X$ (with integrable structure), that is, the subsheaf of non-commutative algebras of $\mathcal{D}_{\mathbb{A}_{z}^{1} \times X}$ generated by $z p^{*} \Theta_{X}$ and $z^{2} \partial_{z}$, where $p: \mathbb{A}_{z}^{1} \times X \rightarrow$ $X$ is the projection. If $\left(x_{1}, \ldots, x_{n}\right)$ are local coordinates on $X$, then $\mathcal{R}_{\mathbb{A}_{z}^{1} \times X}^{\text {int }}$ is locally given by $\mathcal{O}_{\mathbb{A}_{z}^{1} \times X}\left\langle z^{2} \partial_{z}, z \partial_{x_{1}}, \ldots, z \partial_{x_{n}}\right\rangle$. Occasionally, we will also need the sheaf $\mathcal{R}_{\mathrm{A}_{z}^{1} \times X}$, which is generated by $z p^{*} \Theta_{X}$ only, i.e., locally given by $\mathcal{O}_{\mathbb{A}_{z}^{1} \times X}\left\langle z \partial_{x_{1}}, \ldots, z \partial_{x_{n}}\right\rangle$. We let $\operatorname{MHM}(X)$ be the abelian category of algebraic mixed Hodge modules (see $[$ Sai90] $)$ on $X$. We consider moreover the category $\operatorname{MHM}(X, \mathbb{C})$ of complex mixed Hodge modules (see, e.g., [DS13, Definition 3.2.1]). As an example, if $X$ is the algebraic torus $\mathbb{G}_{m}^{d}$ with coordinates $y_{1}, \ldots, y_{d}$, then for any $\alpha=\left(\alpha_{1}, \ldots, \alpha_{d}\right) \in \mathbb{R}^{d}$, the free $\mathcal{O}_{\mathbb{G}_{m}^{d}}$-module of rank 1

$$
\mathcal{O}_{\mathbb{G}_{m}^{d}}^{\alpha}:=\mathcal{D}_{\mathbb{G}_{m}^{d}} /\left(y_{k} \partial_{y_{k}}+\alpha_{k}+1\right)_{k=1, \ldots, d}
$$

(see also Definition 3.7 below) underlies an object in $\operatorname{MHM}\left(\mathbb{G}_{m}^{d}, \mathbb{C}\right)$.

On the other hand, $\operatorname{MTM}(X)$ denotes the abelian category of algebraic mixed twistor $\mathcal{D}$-modules on $X$ (see Moc15a $)$. The category $\operatorname{MTM}^{\text {int }}(X)$ consists of those mixed twistor $\mathcal{D}$-modules where the underlying $\mathcal{R}_{\mathbb{A}_{z}^{1} \times X}$-modules have an integrable structure, i.e., where they are modules over $\mathcal{R}_{\mathbb{A} 1}^{\text {int }} \times X$.

The category $\operatorname{IrmMHM}(X)$, as defined in Sab18a, Def. 2.52], is a certain subcategory of $\operatorname{MTM}^{\text {int }}(X)$ (actually of a variant, called ${ }_{\iota} \operatorname{MTM}^{\text {int }}(X)$, which is shown to be equivalent to $\operatorname{MTM}^{\text {int }}(X)$ in [ibid., 
$\S 1])$ consisting of objects $\widehat{\mathcal{M}}$ that satisfy certain properties. The first of them is that the object ${ }^{\theta} \widehat{\mathcal{M}}$ obtained from $\widehat{\mathcal{M}}$ by substituting $z \theta$ for $z$ is still an object of ${ }_{\iota} \mathrm{MTM}^{\mathrm{int}}\left({ }^{\theta} X\right)$, where ${ }^{\theta} X=\mathbb{G}_{m, \theta} \times X$. That is a remarkable assumption, having its origin in [HS07], but we have to impose a further property, namely that such rescaled objects must have a certain tame behaviour when $\theta$ goes to infinity (or at the origin of $\tau=1 / \theta$, in other words), related to regularity along $\{\tau=0\} \times \mathbb{A}_{z}^{1} \times X$. This two conditions are, essentially, what we ask for a mixed twistor $\mathcal{D}$-module on $X$ to be a mixed twistorrescaled $\mathcal{D}$-module on $X$ (Sab18a, Def. 2.50]). The last condition is a $z$-grading property appearing after identifying $\tau$ with $z$ (cf. [ibid., Def. 2.26]). For more details, see [ibid., $\S 2$ ].

Although the construction of this category may seem rather involved, its main feature is that the $\mathcal{D}_{X}$-module $\mathcal{M}$ associated to an object $\widehat{\mathcal{M}}$ in $\operatorname{IrrMHM}(X)$ carries a good filtration $F_{\bullet}^{\text {irr }} \mathcal{M}$, indexed by $\mathbb{R}$, called the irregular Hodge filtration, which in turn behaves well with respect to several functorial operations. Notice however that this filtration, contrarily to the case of mixed Hodge modules, is not part of the definition of an object of $\operatorname{IrmMHM}(X)$. Very roughly, it can be thought of as defined by the intersection of the canonical $V$-filtration along $\tau=0$ (or rather the filtration induced on the restricted object when $\tau=z$ ) with the $z$-adic filtration on $\widehat{\mathcal{M}}$. In particular, the jumping indices of the irregular Hodge filtration are of the form $\{\alpha+k \mid k \in \mathbb{Z}\}$ for a certain finite set of real numbers $\alpha$.

Acknowledgements. We would like to thank Takuro Mochizuki, Thomas Reichelt and Claude Sabbah for their interest in our work and for many stimulating discussions. We are grateful to the anonymous referee for the many valuable comments and remarks. We thank the Max Planck Institute for Mathematics in the Sciences, where a significant part of the work presented here has been carried out.

\section{Hypergeometric MOdULES AND DIMENSIONAL REDUCTIONS}

In this section we will introduce two different kinds of hypergeometric $\mathcal{D}$-modules: classical and GKZ. We will dedicate more time to classical ones, since they form one of the main objects of study of the paper, and will end by showing the relation between both types, which will be useful for us in the future. We state one of our main results (Theorem 2.13), which is proven in the next section.

Definition 2.1. Let $(n, m) \neq(0,0)$ be a pair of nonnegative integers, and let $\alpha_{1}, \ldots, \alpha_{n}$ and $\beta_{1}, \ldots, \beta_{m}$ be elements of $\mathbb{C}$. The (classical) hypergeometric $\mathcal{D}$-module of type $(n, m)$ associated with the $\alpha_{i}$ and the $\beta_{j}$ is defined as the quotient of $\mathcal{D}_{\mathbb{G}_{m}}$ by the left ideal generated by the so-called hypergeometric operator

$$
\prod_{i=1}^{n}\left(t \partial_{t}-\alpha_{i}\right)-t \prod_{j=1}^{m}\left(t \partial_{t}-\beta_{j}\right) .
$$

We will denote it by $\mathcal{H}\left(\alpha_{1}, \ldots, \alpha_{n} ; \beta_{1}, \ldots, \beta_{m}\right)$, or in an abridged way, $\mathcal{H}\left(\alpha_{i} ; \beta_{j}\right)$.

In this paper we will be mostly concerned with hypergeometric $\mathcal{D}$-modules of type $(n, 0)$.

Remark 2.2. The excluded type $(n, m)=(0,0)$ corresponds to the punctual delta $\mathcal{D}_{\mathbb{G}_{m}}$-module on $\mathbb{G}_{m}$ $\mathcal{H}(\emptyset ; \emptyset)=\mathcal{D}_{\mathrm{G}_{m}} /(1-t)$.

On the other hand, if we denote the Kummer $\mathcal{D}$-module $\mathcal{D}_{\mathbb{G}_{m}} /\left(t \partial_{t}-\eta\right)$ by $\mathcal{K}_{\eta}$, for any fixed complex number $\eta$, then $\mathcal{H}\left(\alpha_{i} ; \beta_{j}\right) \otimes_{\mathcal{O}_{\mathbb{G}_{m}}} \mathcal{K}_{\eta} \cong \mathcal{H}\left(\alpha_{i}+\eta ; \beta_{j}+\eta\right)$. In particular, an overall integer shift of the parameters gives us an isomorphic $\mathcal{D}$-module.

Every hypergeometric $\mathcal{D}$-module has Euler characteristic -1 (cf. Kat90, Lem. 2.9.13]). For $n \neq m$, no hypergeometric $\mathcal{D}$-module of type $(n, m)$ has singularities on $\mathbb{G}_{m}$. If $n>m$ (resp. $m>n$ ), they have a regular singularity at the origin (resp. infinity) and an irregular singularity at infinity (resp. the origin) of irregularity one and slope $1 /|n-m|$ of multiplicity $|n-m|$ (cf. Kat90, Prop. 2.11.9]). For $n=m$, the hypergeometric $\mathcal{D}$-modules of type $(n, n)$ are regular, with singularities only at the origin, infinity and 1.

Proposition 2.3 (Irreducibility). (cf. Kat90, Prop. 2.11.9, 3.2]) Let $\mathcal{H}:=\mathcal{H}\left(\alpha_{i} ; \beta_{j}\right)$ be a hypergeometric $\mathcal{D}$-module. It is irreducible if and only if for any pair $(i, j)$ of indices, $\alpha_{i}-\beta_{j}$ is not an integer. 
Remark 2.4. Assuming the irreducibility, we have a result which is stronger than the second paragraph of Remark 2.2. Namely, the isomorphism class of $\mathcal{H}\left(\alpha_{i} ; \beta_{j}\right)$ depends only on the classes modulo $\mathbb{Z}$ of the $\alpha_{i}$ and the $\beta_{j}$ (point 1 of [Kat90, Prop. 3.2]), so we can choose such parameters on a fundamental domain of $\mathbb{C} / \mathbb{Z}$.

Proposition 2.5 (Rigidity). Let $\mathcal{H}:=\mathcal{H}\left(\alpha_{i} ; \beta_{j}\right)$ be an irreducible hypergeometric $\mathcal{D}$-module of type $(n, m)$, where $n \geq m$, and let $\mathcal{M}$ be another irreducible $\mathcal{D}_{\mathbb{G}_{m}}$-module of Euler-Poincaré characteristic -1 which has no singularities outside $\{0,1, \infty\}$. Assume that

- $\mathcal{H} \otimes \mathbb{C}((t)) \cong \mathcal{M} \otimes \mathbb{C}((t))$.

- $\mathcal{H} \otimes \mathbb{C}((1 / t)) \cong \mathcal{M} \otimes \mathbb{C}((1 / t))$.

- If $n=m$, assume further that $\mathcal{M}$ has a regular singularity at 1 .

In that case, $\mathcal{H}$ and $\mathcal{M}$ are isomorphic.

Proof. $\mathcal{H}$ and $\mathcal{M}$ being irreducible, both of them coincide with the middle extension of their restriction to $\mathbb{G}_{m} \backslash\{1\}$. Assume first that $n=m$, that is, $\mathcal{H}$ is regular. Since $\mathcal{M}$ has characteristic -1 and regular singularities at the origin, one and infinity, by the formula for the Euler characteristic Kat90, Thm. 2.9.9], its formal local monodromy at 1 must be a pseudoreflection. Then we can apply [ibid., Rigidity Thm. 3.5.4] and we are done.

If $n>m, \mathcal{M}$, like $\mathcal{H}$, has a regular singularity at the origin and an irregular singularity at infinity. By the same formula for the Euler characteristic of $j_{++} j^{+} \mathcal{M}$ (denoting by $j$ the inclusion $\mathbb{G}_{m} \backslash\{1\} \hookrightarrow \mathbb{G}_{m}$ ), $\mathcal{M}$ cannot have more singularities in $\mathbb{P}^{1}$ apart from zero and infinity. In that case we apply [ibid., Rigidity Thm. bis 3.7.3].

Consider the case in which $\mathcal{H}=\mathcal{H}\left(\alpha_{i} ; \beta_{j}\right)$ is of type $(n, n)$, i.e., such that it is regular. From the rigidity property of the last Proposition, one concludes by [Sim90, Cor. 8.1] that the restriction of $\mathcal{H}$ to $\mathbb{G}_{m} \backslash\{1\}$ underlies a complex polarizable variation of Hodge structures. The next statement, conjectured by Corti and Golyshev (cf. [CG11, Conj. 1.4]) and proved by Fedorov (see [Fed18, Thm. 1]), gives important information on its Hodge invariants.

Proposition 2.6 (Hodge numbers (regular case)). Let $\mathcal{H}=\mathcal{H}\left(\alpha_{i} ; \beta_{j}\right)$ be an irreducible hypergeometric $\mathcal{D}$-module of type $(n, n)$. Assume that the $\alpha_{i}$ and the $\beta_{j}$ are increasingly ordered real numbers, lying in the interval $[0,1)$. Set

$$
\rho(k)=\left|\left\{i=1, \ldots, n: \beta_{i}<\alpha_{k}\right\}\right|-k,
$$

for $k=1, \ldots, n$. Then the Hodge numbers of $\mathcal{H}$ are, up to an overall shift,

$$
h^{p}=\left|\rho^{-1}(p)\right|=|\{k=1, \ldots, h: \rho(k)=p\}| .
$$

This last result is the analogous one, in the regular case, to Theorem 4.7, and served as the main motivation to start this project. Notice that this formula slightly differs from the one given in [Fed18]. This is because Fedorov considers the dual connection to ours by working with the space of solutions instead of with that of horizontal sections (cf. [Mar18, p. 10, proof of Lem. 3.6]). Now that we have seen part of the behaviour of classical hypergeometric $\mathcal{D}$-modules, let us continue with the other family mentioned above.

Definition 2.7. Let $n \geq m$ two positive integers, and let $d=n-m$. Let $\beta \in \mathbb{C}^{d}$ be a vector and let $A=\left(a_{i j}\right) \in \mathrm{M}(d \times n, \mathbb{Z})$ be an integer matrix. Consider the $n$-dimensional torus $\mathbb{G}_{m}^{n}$ with coordinates $\lambda_{1}, \ldots, \lambda_{n}$. We define the Euler operators $E_{i}=\sum_{j} a_{i j} \lambda_{j} \partial_{\lambda_{j}}$, for $i=1, \ldots, d$, and the toric ideal

$$
I_{A}:=\left(\partial_{\lambda}^{u}-\partial_{\lambda}^{v}: A u=A v\right) \subset \mathbb{C}\left[\partial_{\lambda_{1}}, \ldots, \partial_{\lambda_{n}}\right] \subset D_{\mathbb{A}^{n}} .
$$

Then, the GKZ-hypergeometric $\mathcal{D}$-module (or system) is

$$
\mathcal{M}_{A}^{\beta}:=\mathcal{D}_{\mathbb{G}_{m}^{n}} /\left(\mathcal{I}_{A}+\left(E_{i}-\beta_{i}: i=1, \ldots, d\right)_{i=1, \ldots, d}\right),
$$

where $\mathcal{I}_{A}$ is the sheafified version of the toric ideal $I_{A}$.

Usually the module $\mathcal{M}_{A}^{\beta}$ is defined to be an element in $\operatorname{Mod}\left(\mathcal{D}_{\mathrm{A}^{n}}\right)$. However, we will later work only with the restriction of such an object to $\mathbb{G}_{m}^{n}$. In order to avoid the usage of the functor $j^{+}$(where $j: \mathbb{G}_{m}^{n} \hookrightarrow \mathbb{A}^{n}$ ) each time that we need to refer to $\mathcal{M}_{A}^{\beta}$, we use this slightly non-standard definition. 
A classical hypergeometric $\mathcal{D}$-module can be considered as a dimensional reduction of a certain GKZ-system. We will describe this procedure in some more detail now, because it allows us to apply some of the many known results on GKZ-systems to classical hypergeometric $\mathcal{D}$-modules.

Proposition 2.8. Let $m$ be a positive integer. Let $A \in \mathrm{M}((m-1) \times m, \mathbb{Z})$ be an integer matrix of rank $m-1$ and take $\kappa \in \mathbb{C}^{m}$ such that $\kappa_{1}=0$. Consider the inclusion $\iota: \mathbb{G}_{m} \hookrightarrow \mathbb{G}_{m}^{m}$ given by $t \mapsto(t, 1, \ldots, 1)$, and let $B=\left(b_{1}, \ldots, b_{m}\right)^{t} \in \mathbb{Z}^{m}$ be a Gale dual of $A$, that is, an integer column matrix which generates $\operatorname{ker}_{\mathbb{Q}} A$. Assume moreover that $b_{1}=1$. Put

$$
\eta:=\prod_{i=1}^{m} b_{i}^{b_{i}} .
$$

Then we have $h_{\eta}^{+} \mathcal{H}\left(\alpha_{i} ; \beta_{j}\right) \cong \iota^{+} \mathcal{M}_{A}^{A \kappa}$, where $h_{\eta}$ is the automorphism of $\mathbb{G}_{m}$ given by $t \mapsto \eta t$, and the unordered sets of parameters $\alpha_{i}$ and $\beta_{j}$, counted with multiplicities, are

$$
\begin{gathered}
\left(\alpha_{i}\right)=\left(\frac{k-\kappa_{j}}{b_{j}}: b_{j}>0, k=0, \ldots, b_{j}-1\right), \\
\left(\beta_{i}\right)=\left(\frac{k-\kappa_{j}}{b_{j}}: b_{j}<0, k=0, \ldots,-b_{j}-1\right) .
\end{gathered}
$$

Proof. On one hand, $\mathcal{M}_{A}^{A \kappa}$ is not only a GKZ-hypergeometric $\mathcal{D}$-module, but also the restriction to $\mathbb{G}_{m}^{n}$ of a lattice basis binomial $\mathcal{D}_{\mathbb{A}^{m}}$-module (cf. [BMW18, Def. 1.2], noting that the assumption on the columns of $B$ is not needed for the definition). This is because $A$ being of rank $m-1$ implies that the toric ideal $I_{A}$ coincides with the lattice basis ideal

$$
I(B):=\left(\partial_{\lambda}^{w_{+}}-\partial_{\lambda}^{w_{-}}: w=w_{+}-w_{-} \text {is a column of } B\right) .
$$

(In fact this holds for any complete intersection ideal, but here the argument is simpler.)

On the other hand, the expression we have given for the parameters of $\mathcal{H}\left(\alpha_{i} ; \beta_{j}\right)$ follows from applying the definition of Horn hypergeometric $\mathcal{D}$-modules given in [ibid., Def. 1.1] for a column matrix, up to the same caveat above about the columns on $B$ (in fact normalized ones, but all kinds of Horn $\mathcal{D}$-modules defined in loc. cit. are equal once restricted to the torus $\left.\mathbb{G}_{m, t}\right)$, and comparing it with Definition 2.1.

Now let $j: \mathbb{G}_{m} \hookrightarrow \mathbb{A}^{1}$ be the canonical inclusion. The isomorphism given in [ibid., Thm. 1.4] relates lattice basis binomial $\mathcal{D}$-modules to Horn hypergeometric ones. However, due to the previous discussions, we obtain the isomorphism in the statement just by applying $j^{+}$to both sides of it.

Note that the choice of $\kappa_{1}$ and $b_{1}$ in the statement of the Proposition force $\alpha_{1}$ to vanish. However, by Remark 2.2 , any other hypergeometric $\mathcal{D}$-module can be built from one of this form just by tensoring with a suitable Kummer $\mathcal{D}$-module.

In our study of Hodge theoretic properties of hypergeometric $\mathcal{D}_{\mathbb{G}_{m}}$-modules below, we need to go in some sense in the opposite direction: Given sets $\left\{\alpha_{i}\right\},\left\{\beta_{j}\right\}$, we would like to determine a matrix $A$ and a parameter vector $\beta$ such that the module $\mathcal{H}\left(\alpha_{i} ; \beta_{j}\right)$ can be obtained as an inverse image of the GKZ-system $\mathcal{M}_{A}^{\beta}$. Although such a pair $(A, \beta)$ is not unique, a systematic way of constructing it can be formulated as follows.

Corollary 2.9. Let $\mathcal{H}\left(\alpha_{i} ; \beta_{j}\right)$ be a hypergeometric $\mathcal{D}_{\mathbb{G}_{m}}$-module of type $(n, m)$ with $n>0$ and $\alpha_{1}=0$. Let $A \in \mathrm{M}((m+n-1) \times(n+m), \mathbb{Z})$ given by

$$
A=\left(\begin{array}{c|c|c}
\underline{1}_{m} & \underline{0}_{m \times(n-1)} & \operatorname{Id}_{m} \\
\hline \underline{1}_{n-1} & -\operatorname{Id}_{n-1} & \underline{0}_{(n-1) \times m}
\end{array}\right),
$$

and let $\beta=\left(\beta_{1}, \ldots, \beta_{m}, \alpha_{2}, \ldots, \alpha_{n}\right)^{\mathrm{t}}$. (Here and later in this paper, we write $\underline{x}_{k}$ for a column vector with $k$ rows, where all entries contain the value $x$.) Let $\iota: \mathbb{G}_{m} \rightarrow \mathbb{G}_{m}^{n+m}$, given by $t \mapsto(t, 1 \ldots, 1)$. Then

$$
\mathcal{H}\left(\alpha_{i} ; \beta_{j}\right) \cong \iota^{+} \mathcal{M}_{A}^{\beta} \text {. }
$$

Proof. The statement is an easy consequence of the Proposition, taking $B=(1, \stackrel{(n)}{.}, 1,-1, \stackrel{(m)}{.},-1)^{\mathrm{t}}$ and $\kappa=\left(0,-\alpha_{2}, \ldots,-\alpha_{n}, \beta_{1}, \ldots, \beta_{m}\right)^{\mathrm{t}}$. 
As indicated before, we will see later that the restriction $\alpha_{1}=0$ is not as strong as it may appear: By tensoring a given hypergeometric $\mathcal{D}$-module with an appropriate Kummer module, we can always reach this assumption.

We will end this section by explaining how the above construction of GKZ-systems and the dimensional reduction to hypergeometric $\mathcal{D}_{\mathbb{G}_{m, t}}$-modules can be understood at the level of $\mathcal{R}$-modules. Recall (see the introduction) that for a smooth algebraic variety $X$ with local coordinates $\left(x_{1}, \ldots, x_{n}\right)$ the sheaf $\mathcal{R}_{\mathbb{A}_{z}^{1} \times X}^{\text {int }}$ is the subsheaf of $\mathcal{D}_{\mathbb{A}_{z}^{1} \times X}$ locally generated by $z^{2} \partial_{z}$ and $\left(z \partial_{x_{i}}\right)_{i=1, \ldots, n}$.

Definition 2.10. Let $n \geq m$ two positive integers, and let $d=n-m$. Let $\beta \in \mathbb{C}^{d}$ be a vector and let $A=\left(a_{i j}\right) \in \mathrm{M}(d \times n, \mathbb{Z})$ be an integer matrix. Consider the affine space $\mathbb{G}_{m}^{n}$ with coordinates $\lambda_{1}, \ldots, \lambda_{n}$, and let $\mathbb{L} \subset \mathbb{Z}^{n}$ be the kernel of the linear map $\mathbb{Z}^{n} \rightarrow \mathbb{Z}^{d}$ given by left multiplication by the matrix $A$, whose elements will be denoted by $\underline{l}=\left(l_{1}, \ldots, l_{n}\right)$. Then, the GKZ-hypergeometric $\mathcal{R}$-module is

where $\beta_{0} \in \mathbb{C}$ and $\mathcal{I}$ is generated by

$$
\widehat{\mathcal{M}}_{A}^{\left(\beta_{0}, \beta\right)}:=\mathcal{R}_{\mathbb{A}_{z}^{1} \times \mathbb{G}_{m}^{n}}^{\mathrm{int}} / \mathcal{I}
$$

$$
\begin{gathered}
\prod_{j: l_{j}>0}\left(z \partial_{\lambda_{j}}\right)^{l_{j}}-\prod_{j: l_{j}<0}\left(z \partial_{\lambda_{j}}\right)^{-l_{j}}, \underline{l} \in \mathbb{L} \\
z^{2} \partial_{z}+\lambda_{1} z \partial_{\lambda_{1}}+\ldots+\lambda_{n} z \partial_{\lambda_{n}}-z \beta_{0} \\
\sum_{j=1}^{n} a_{k j} \lambda_{j} z \partial_{\lambda_{j}}-z \beta_{k}, k=1, \ldots, d .
\end{gathered}
$$

Note that we can recover the GKZ-hypergeometric $\mathcal{D}$-module $\mathcal{M}_{A}^{\beta}$ from Definition 2.7 by restricting $\widehat{\mathcal{M}}_{A}^{\left(\beta_{0}, \beta\right)}$ to $z=1$. In the special case of our original matrix from Corollary 2.9 the generators of $\mathcal{I}$ are

$$
\begin{gathered}
\left(z \partial_{\lambda_{1}}\right) \cdot \ldots \cdot\left(z \partial_{\lambda_{n}}\right)-\left(z \partial_{\lambda_{n+1}}\right) \cdot \ldots \cdot\left(z \partial_{\lambda_{n+m}}\right) \\
z^{2} \partial_{z}+\lambda_{1} z \partial_{\lambda_{1}}+\ldots+\lambda_{n+m} z \partial_{\lambda_{n+m}}-z \beta_{0} \\
\lambda_{1} z \partial_{\lambda_{1}}+\lambda_{n+i} z \partial_{\lambda_{n+i}}-z \beta_{i}, i=1, \ldots, m \\
\lambda_{1} z \partial_{\lambda_{1}}-\lambda_{i} z \partial_{\lambda_{i}}+z \alpha_{i}, i=2, \ldots, n
\end{gathered}
$$

Moreover, we must also consider the corresponding $\mathcal{R}$-module for hypergeometric $\mathcal{D}$-modules. Both kinds of $\mathcal{R}$-modules will play a significant role in the proof of Theorem 2.13 .

Definition 2.11. Let $(n, m) \neq(0,0)$ be a pair of natural numbers, and let $\alpha_{1}, \ldots, \alpha_{n}$ and $\beta_{1}, \ldots, \beta_{m}$ be elements of $\mathbb{C}$. The (classical) hypergeometric $\mathcal{R}$-module (of type $(n, m)$ ) associated with the $\alpha_{i}$ and the $\beta_{j}$, denoted by $\widehat{\mathcal{H}}\left(\alpha_{i} ; \beta_{j}\right)$, is defined as the quotient of $\mathcal{R}_{\mathbb{A}_{z}^{1} \times \mathbb{G}_{m, t}}^{\text {int }}$ by the left ideal generated by

$$
P=z^{2} \partial_{z}+(n-m) t z \partial_{t}+\gamma z \text { and } H=\prod_{i=1}^{n} z\left(t \partial_{t}-\alpha_{i}\right)-t \prod_{j=1}^{m} z\left(t \partial_{t}-\beta_{j}\right),
$$

where $\gamma=-\sum_{i=1}^{n} \alpha_{i}+\sum_{j=1}^{m} \beta_{j}$.

The choice of the operator $P$ may seem odd, but as we will see, it is indeed very natural. In fact, we have the following extension of Corollary 2.9 to the realm of $\mathcal{R}$-modules.

Lemma 2.12. Let $\widehat{\mathcal{H}}\left(\alpha_{i} ; \beta_{j}\right)$ be a classical hypergeometric $\mathcal{R}_{\mathbb{A}_{z}^{1} \times \mathbb{G}_{m, t}}$-module of type $(n, m)$ with $n>0$ and $\alpha_{1}=0$. Let $A \in \mathrm{M}((m+n-1) \times(n+m), \mathbb{Z}), \beta \in \mathbb{C}^{n+m-1}$ and $\iota: \mathbb{G}_{m, t} \hookrightarrow \mathbb{G}_{m}^{n+m}$ be as in the statement of Corollary 2.9. Then

$$
\widehat{\mathcal{H}}\left(\alpha_{i} ; \beta_{j}\right) \cong \iota^{+} \widehat{\mathcal{M}}_{A}^{(0, \beta)} .
$$

Proof. The inverse image functor in the category of $\mathcal{R}$-modules is induced by the usual inverse image functor of $\mathcal{O}$-modules, $\left(\operatorname{id}_{\mathrm{A}_{z}^{1}} \times \iota\right)^{*}$ in this case ( $\operatorname{cf}$. Moc15a, $\left.\left.\S 2.1 .6 .2\right]\right)$. Then it is easy to see that

$$
\widehat{\mathcal{H}}\left(\alpha_{i} ; \beta_{j}\right) \cong \iota^{+} \widehat{\mathcal{M}}_{A}^{(0, \beta)} .
$$


Namely, we replace $z \lambda_{i} \partial_{\lambda_{i}}$ by $z \lambda_{1} \partial_{\lambda_{1}}-z \alpha_{i}$ if $i=2, \ldots, n$ or by $-z \lambda_{1} \partial_{\lambda_{1}}+z \beta_{i-n}$, if $i=n+1, \ldots, n+m$. Since we can invert $\lambda_{i}$ in $\mathcal{R}_{\mathbb{A}_{z}^{1} \times \mathbb{G}_{m}^{n}}^{\text {int }}$, we present $\widehat{\mathcal{M}}_{A}^{(0, \beta)}$ as the $\mathcal{O}_{\mathbb{G}_{m}^{n+m}}$-module $\mathcal{O}_{\mathbb{G}_{m}^{n+m}}\left\langle z^{2} \partial_{z}, z \lambda_{1} \partial_{\lambda_{1}}\right\rangle / \mathcal{J}$, where $\mathcal{J}$ is generated by

$\lambda_{n+1} \cdot \ldots \cdot \lambda_{n+m} \prod_{i=1}^{n} z\left(\lambda_{1} \partial_{\lambda_{1}}-\alpha_{i}\right)-(-1)^{m} \lambda_{1} \cdot \ldots \cdot \lambda_{n} \prod_{i=1}^{m} z\left(\lambda_{1} \partial_{\lambda_{1}}-\beta_{i}\right)$ and $z^{2} \partial_{z}+(n-m) z \lambda_{1} \partial_{\lambda_{1}}+\gamma z$.

Now the inverse image by $\iota$ amounts simply to set $\lambda_{1}=t$ and $\lambda_{i}=1$ for $i=2, \ldots, n+m$ in the generators of the ideal, from which the desired isomorphism follows, up to multiplying $t$ by -1 .

We can formulate at this point one of the main results of this paper. Its full proof will occupy the entire next section.

Theorem 2.13. Let $\alpha_{1}, \ldots, \alpha_{n}$ be real numbers, belonging to the interval $[0,1)$ and increasingly ordered, and let $\gamma=-\sum_{i=1}^{n} \alpha_{i}$. Then the $\mathcal{R}_{\mathbb{A}_{z}^{1} \times \mathbb{G}_{m, t}}$-module $\widehat{\mathcal{H}}:=\widehat{\mathcal{H}}\left(\alpha_{i}, \emptyset\right)=\mathcal{R}_{\mathbb{A}_{z}^{1}}^{\text {int }} \times \mathbb{G}_{m, t} /(P, H)$, where

$$
P=z^{2} \partial_{z}+n t z \partial_{t}+\gamma z \text { and } H=\prod_{i=1}^{n} z\left(t \partial_{t}-\alpha_{i}\right)-t,
$$

underlies an irregular Hodge module, i.e., an object of $\operatorname{IrrMHM}\left(\mathbb{G}_{m, t}\right)$. It is the unique irregular Hodge module whose associated $\mathcal{D}_{\mathbb{G}_{m, t}}$-module is $\mathcal{H}\left(\alpha_{i} ; \emptyset\right)$. Moreover, $\widehat{\mathcal{H}}$ can be extended in a unique way to an $\mathcal{R}_{\mathbb{A}_{z}^{1} \times \mathbb{P}^{1}}^{\text {int }}$-module, $\widehat{\mathcal{H}}_{p r}$, such that it underlies an object of $\operatorname{IrrMHM}\left(\mathbb{P}^{1}\right)$.

Proof of unicity. We will give here a proof of the two unicity statements in the above theorem, postponing the proof of the main statement to page 25 below.

Consider any twistor $\mathcal{D}$-module $\widehat{\mathcal{H}}^{\prime}$ on $\mathbb{G}_{m, t}$ whose underlying $\mathcal{D}_{\mathbb{G}_{m, t}}$-module is $\mathcal{H}$. Since the functor $\Xi_{\mathrm{DR}}$ is faithful by Moc15a, Rem. 7.2.9], we have an injection of Hom groups

$$
\operatorname{Hom}_{\operatorname{MTM}\left(\mathbb{G}_{m, t}\right)}\left(\widehat{\mathcal{H}}, \widehat{\mathcal{H}}^{\prime}\right) \hookrightarrow \operatorname{Hom}_{\mathcal{D}_{\mathbb{G}_{m, t}}}(\mathcal{H}, \mathcal{H}) .
$$

But $\mathcal{H}$ is irreducible, so its only endomorphism is the identity and then, a twistor $\mathcal{D}$-module underlying $\mathcal{H}$ is unique, if it exists.

On the other hand, let $j: \mathbb{G}_{m, t} \hookrightarrow \mathbb{P}^{1}$ be the canonical inclusion and consider the $\mathcal{D}_{\mathbb{P}^{1} \text {-module }}$

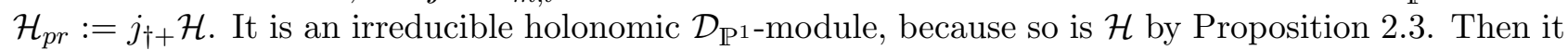
gives rise to a unique pure integrable twistor $\mathcal{D}$-module $\widehat{\mathcal{H}}_{p r}$ on $\mathbb{P}^{1}$ by Moc11, Thm. 1.4.4] and [Sab18a,

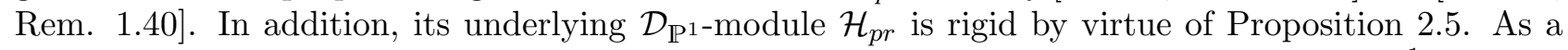
consequence, we can invoke [Sab18a, Thm. 0.7] and claim that such twistor $\mathcal{D}$-module on $\mathbb{P}^{1}$ is in fact an object of $\operatorname{IrrMHM}\left(\mathbb{P}^{1}\right)$. Take now $\widehat{\mathcal{H}}^{\prime}:=j^{+} \widehat{\mathcal{H}}_{p r}$, which is an irregular mixed Hodge module whose underlying $\mathcal{D}_{\mathbb{G}_{m, t}}$-module is $\mathcal{H}$, by Moc15a, Prop. 14.1.24]. The we must have, as was just shown, $\widehat{\mathcal{H}}^{\prime} \cong \widehat{\mathcal{H}}$, so that the extension $\widehat{\mathcal{H}}_{p r}$ of $\hat{\mathcal{H}}$ is unique, as claimed.

The main point in the above theorem is that $\widehat{\mathcal{H}}$ underlies an irregular mixed Hodge module. Since the proof of this fact is rather long, and will be carried out in the next section through various intermediate results, we would like to orient the reader by giving here an overview of these steps. We will restrict the sketch to the case where $\alpha_{1}=0$, this is also the first (and main) step in the actual proof below on page 25. The general case can be rather easily deduced from this special one by considering Kummer $\mathcal{D}$ - resp. $\mathcal{R}$-modules.

The first point is to realise $\widehat{\mathcal{H}}$ in a geometric way. For this purpose, consider the following two families of Laurent polynomials

$$
f\left(y_{1}, \ldots, y_{n-1}, \lambda_{1}, \ldots, \lambda_{n}\right):=-\lambda_{1} \cdot y_{1} \cdot \ldots \cdot y_{n-1}-\frac{\lambda_{2}}{y_{1}}-\ldots-\frac{\lambda_{n}}{y_{n-1}}
$$

and

$$
{ }^{\prime} f\left(y_{1}, \ldots, y_{n-1}, t\right):=-t \cdot y_{1} \cdot \ldots \cdot y_{n-1}-\frac{1}{y_{1}}-\ldots-\frac{1}{y_{n-1}}
$$


where $y_{k}, \lambda_{i}, t \in \mathbb{G}_{m}$. Write $\iota: \mathbb{G}_{m, t} \hookrightarrow \mathbb{G}_{m}^{n}, t \mapsto(t, 1, \ldots, 1)$, so that we have the cartesian diagram

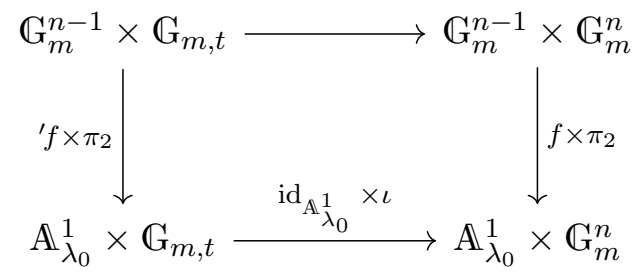

where we denote the coordinate on the affine line corresponding to the value of $f$ resp. of ' $f$ by $\lambda_{0}$, for reasons that will become clear later.

We consider the so-called twisted cohomology groups associated to the morphisms $f$ and ' $f$. It can be shown (see Proposition 3.8 below) that

$$
\widehat{\mathcal{M}}_{A}^{(0, \alpha)} \cong \mathcal{H}^{n-1}\left(\pi_{2, *} \Omega_{\mathbb{G}_{m}^{n-1} \times \mathbb{G}_{m}^{n} / \mathbb{G}_{m}^{n}}^{\bullet+d}[z], z(d-\kappa(\alpha) \wedge)-d f \wedge\right),
$$

where $(0, \alpha)=\left(0, \alpha_{1}, \alpha_{2}, \ldots, \alpha_{n}\right)=\left(0,0, \alpha_{2}, \ldots, \alpha_{n}\right)$, where $\kappa(\alpha)=\sum_{j=1}^{n-1} \alpha_{j+1} d y_{j} / y_{j}$ and $A$ is the matrix from Corollary 2.9 for the case $m=0$. Moreover, since the twisted cohomology groups involve complexes of relative differential forms, we have

$$
\begin{aligned}
& \iota^{+} \mathcal{H}^{n-1}\left(\pi_{2, *} \Omega_{\mathbb{G}_{m}^{n-1} \times \mathbb{G}_{m}^{n} / \mathbb{G}_{m}^{n}}^{\bullet+d}[z], z(d-\kappa(\alpha) \wedge)-d f \wedge\right) \\
\cong & \mathcal{H}^{n-1}\left(\pi_{2, *} \Omega_{\mathbb{G}_{m}^{n-1} \times \mathbb{G}_{m, t} / \mathbb{G}_{m, t}}^{\bullet}[z], z(d-\kappa(\alpha) \wedge)-d^{\prime} f \wedge\right),
\end{aligned}
$$

so that by using Lemma 2.12 we obtain an isomorphism of $\mathcal{R}_{\mathbb{A}_{z}^{1} \times \mathbb{G}_{m, t}}^{\mathrm{int}}$-modules

$$
\widehat{\mathcal{H}} \cong \mathcal{H}^{n-1}\left(\pi_{2, *} \Omega_{\mathbb{G}_{m}^{n-1} \times \mathbb{G}_{m, t} / \mathbb{G}_{m, t}}[z], z(d-\kappa(\alpha) \wedge)-d^{\prime} f \wedge\right) .
$$

As a second step, we will realize the right hand side of the above isomorphism in a different way. Namely, write ${ }^{\prime} \varphi:=\left({ }^{\prime} f, \pi_{2}\right): \mathbb{G}_{m}^{n-1} \times \mathbb{G}_{m, t} \rightarrow \mathbb{A}_{\lambda_{0}}^{1} \times \mathbb{G}_{m, t}$ and consider the direct image complex ${ }^{\prime} \varphi_{+} \mathcal{O}_{\mathbb{G}_{m}^{n-1}}^{\alpha} \in \mathrm{D}_{\mathrm{rh}}^{\mathrm{b}}\left(\mathcal{D}_{\mathbb{A}_{\lambda_{0}}^{1} \times \mathbb{G}_{m, t}}\right)$, where $\mathcal{O}_{\mathbb{G}_{m}^{n-1}}^{\alpha}=\mathcal{D}_{\mathbb{G}_{m}^{n-1}} /\left(y_{j} \partial_{y_{j}}+\alpha_{j+1}+1\right)_{j=1, \ldots, n-1}$. We are interested in the top cohomology of this complex. Standard techniques for the calculation of direct images of $\mathcal{D}$-modules show that it is given by

$$
M:=\frac{\pi_{2, *} \Omega_{\mathbb{G}_{m}^{n-1} \times \mathbb{G}_{m, t} / \mathbb{G}_{m, t}}^{n-1}\left[\partial_{\lambda_{0}}\right]}{\left(d-\partial_{\lambda_{0}} \cdot d^{\prime} f \wedge\right) \pi_{2, *} \Omega_{\mathbb{G}_{m}^{n-2} \times \mathbb{G}_{m, t} / \mathbb{G}_{m, t}}\left[\partial_{\lambda_{0}}\right]}
$$

We will use a variant of the Fourier-Laplace transformation (called localized partial Fourier-Laplace transformation, and denoted by $\mathrm{FL}_{\mathbb{G}_{m, t}}^{\text {loc }}$, see Definition 3.10 and Definition 3.11 below) exchanging the operator $\partial_{\lambda_{0}}$. into $z^{-1}$, the operator $\lambda_{0}$. into $z^{2} \partial_{z}$, and localizing along $z=\infty$. Then we have an isomorphism of $\mathcal{D}_{\mathbb{A}_{z}^{1} \times \mathbb{G}_{m, t}}$-modules

$$
\mathrm{FL}_{\mathbb{G}_{m, t}}^{\operatorname{loc}}(M)=\frac{\pi_{2, *} \Omega_{\mathbb{G}_{m}^{n-1} \times \mathbb{G}_{m, t} / \mathbb{G}_{m, t}}^{n-1}\left[z^{ \pm}\right]}{\left(z \cdot d-d^{\prime} f \wedge\right) \pi_{2, *} \Omega_{\mathbb{G}_{m}^{n-1} \times \mathbb{G}_{m, t} / \mathbb{G}_{m, t}}^{n-2}\left[z^{ \pm}\right]} \cong \widehat{\mathcal{H}}\left[z^{ \pm}\right] \supset \widehat{\mathcal{H}}
$$

One of the main points of the proof in the next section is to give a good description of the image of $\widehat{\mathcal{H}}$ inside $\mathrm{FL}_{\mathbb{G}_{m, t}}^{\text {loc }}(M)$ under this isomorphism. One such description is given by the isomorphism of $\mathcal{R}_{\mathbb{A}_{z}^{1} \times \mathbb{G}_{m, t}}^{\text {int }}$-modules in the displayed formula (1). However, we cannot, a priori, obtain any Hodge theoretic information on $\widehat{\mathcal{H}}$ from (1). On the other hand, we know that $M$ underlies an algebraic complex mixed Hodge module on $\mathbb{A}_{\lambda_{0} \times \mathbb{G}_{m, t}}^{1}$ (since it is the direct image of such an object on $\mathbb{G}_{m}^{n-1}$ ), and hence it comes equipped with a certain good filtration $F_{\bullet}^{H} M$ (the Hodge filtration). There is a general procedure, explained below in Definition 3.20 and Lemma 3.21, which constructs, given a 
filtered $\mathcal{D}_{\mathbb{A}_{\lambda_{0}} \times \mathbb{G}_{m, t}}$-module $\left(N, F_{\bullet}\right)$, a $\mathcal{R}_{\mathbb{A}_{z}^{1} \times \mathbb{G}_{m, t}}^{\text {int }}$-module called $G_{0}^{F} \mathrm{FL}_{\mathbb{G}_{m, t}}^{\text {loc }} N$ such that its localisation $G_{0}^{F} \mathrm{FL}_{\mathbb{G}_{m, t}}^{\text {loc }} N \otimes_{\mathcal{O}_{\mathbb{A}_{z}^{1} \times \mathbb{G}_{m, t}}} \mathcal{O}_{\mathrm{A}_{z}^{1} \times \mathbb{G}_{m, t}}\left[z^{-1}\right]$ equals $\mathrm{FL}_{\mathbb{G}_{m, t}}^{\text {loc }} N$. Then we show in Theorem 3.24 that

$$
\widehat{\mathcal{H}} \cong G_{0}^{F^{H}} \mathrm{FL}_{\mathbb{G}_{m, t}}^{\operatorname{loc}} M
$$

up to a shift of the Hodge filtration. Actually, the proof is not that direct, since we have to identify $\mathrm{FL}_{\mathbb{G}_{m, t}}^{\text {loc }}(M)$ with the localized partial Fourier-Laplace transformation of some other $\mathcal{D}_{\mathbb{A}_{\lambda_{0}}^{1} \times \mathbb{G}_{m, t}}$-module (called $M_{\dagger+}$ ), which underlies a pure polarizable Hodge module. It is constructed by taking a compactification of ' $f$, i.e., a projective morphism defined on a quasi-projective (usually singular) variety constructed from the toric compactification of $\mathbb{G}_{m}^{n-1}$ inside $\mathbb{P}^{n}$. Then $M_{\dagger+}$ is obtained as the direct image under this projective morphism of a certain intersection cohomology module. Now it is known (see Sab18a, Cor. 0.5]) that if $M_{\dagger+}$ underlies a pure polarizable Hodge module, the $\mathcal{R}_{\mathbb{A}_{z}^{1} \times \mathbb{G}_{m, t}}^{\text {int }}$-module $G_{0}^{F^{H}} \mathrm{FL}_{\mathbb{G}_{m, t}}^{\text {loc }} M_{\dagger+}$ underlies an irregular Hodge module, which finishes the proof. Notice that the proof of the identification $\mathrm{FL}_{\mathbb{G}_{m, t}}^{\text {loc }}(M) \cong \mathrm{FL}_{\mathbb{G}_{m, t}}^{\text {loc }}\left(M_{\dagger+}\right)$ is derived from a similar isomorphism for the direct images of the morphisms $\varphi=\left(f, \mathrm{id}_{\mathbb{G}_{m}^{n}}\right)$ resp. its compactification, rather than for ' $\varphi$, and is done via the formalism of Radon transformations for regular holonomic $\mathcal{D}$-modules (in the same way as in Rei14 and RS17, RS15a).

\section{Hodge modules and Fourier-Laplace transformation}

Let $\alpha_{1}, \ldots, \alpha_{n}$ be real numbers, and consider the hypergeometric $\mathcal{D}_{\mathbb{G}_{m}}$-module $\mathcal{H}=\mathcal{H}\left(\alpha_{i} ; \emptyset\right)$. As we have mentioned before, the goal of this section is to prove Theorem 2.13 above, showing that the $\mathcal{R}$-module $\widehat{\mathcal{H}}:=\widehat{\mathcal{H}}\left(\alpha_{i} ; \emptyset\right)$ from Definition 2.11 underlies an object of $\operatorname{IrMHM}\left(\mathbb{G}_{m}\right)$ (the abelian category of exponential Hodge modules as defined in Sab18a, see the introduction). We have already indicated several times that we will use the GKZ-hypergeometric $\mathcal{R}$-module $\widehat{\mathcal{M}}_{A}^{(0, \alpha)}$ for the matrix $A$ from corollary 2.9, but where $m=0$. However, we will start with a more general situation, and specify the assumptions we need when going on with the proof.

Let $d<n$ be two positive integers, and take a parameter vector $\beta \in \mathbb{C}^{d}$. For the Hodge theoretic questions we are interested in, only real parameter vectors are relevant, but we will work with this more general setting until Remark 3.17 below. Let $A=\left(\underline{a}_{1}, \ldots, \underline{a}_{n}\right) \in \mathrm{M}(d \times n, \mathbb{Z})$ be an integer matrix satisfying the following:

\section{Assumptions 3.1.}

(i) $\mathbb{Z} A=\mathbb{Z}^{d}$, here $\mathbb{Z} A:=\sum_{i=1}^{n} \mathbb{Z} \underline{a}_{i}$,

(ii) Let $\Delta:=\operatorname{Conv}\left(\underline{a}_{1}, \ldots, \underline{a}_{n}\right)$ be the convex hull in $\mathbb{R}^{d}$ of the vectors given by the columns of the matrix $A$. Then for any proper face $\Gamma \subset \Delta$ we require that the set $\left\{\underline{a}_{i} \mid \underline{a}_{i} \in \Gamma\right\}$ is part of a Q-basis of $\mathbb{Q}^{d}$.

(iii) The origin lies in the interior of $\Delta$.

Remark 3.2. (i) These assumptions are in particular satisfied if $\underline{a}_{1}, \ldots, \underline{a}_{n}$ are the primitive integral generators of the rays of the fan $\Sigma$ defining a toric Fano orbifold $X_{\Sigma}$, since in this case it is known (see [CK99, Lem. 3.2.1 and $\S 3.5]$ ) that $\Sigma$ is the union of the cones over the proper faces of $\Delta$, so that assumption (ii) is satisfied by the fact that the cones of $\Sigma$ are simplicial. Moreover, for Fano toric varieties the origin is the only integer point in the interior of $\Delta$, so (iii) obviously holds. In any case, we see that these conditions are satisfied for the example

$$
A=\left(\begin{array}{cccccc}
1 & -1 & 0 & 0 & \ldots & 0 \\
1 & 0 & -1 & 0 & \ldots & 0 \\
\vdots & \ldots & & & & \\
1 & 0 & 0 & 0 & \ldots & -1
\end{array}\right) \in \mathrm{M}((n-1) \times n, \mathbb{Z}),
$$

since these are the generators of the rays of the fan of $\mathbb{P}^{n-1}$. As we have seen in Corollary 2.9 , this is the matrix we have to look at when we want to express the classical hypergeometric $\mathcal{D}$-module of type $(n, 0)$ and where $\alpha_{0}=0$ as an inverse image of the GKZ-system $\mathcal{M}_{A}^{\beta}$ where $\beta=\alpha:=\left(\alpha_{2}, \ldots, \alpha_{n}\right)^{\mathrm{t}} \in \mathbb{R}^{n-1}$. 
(ii) Assumption (iii) from above implies in particular that there is a relation $\underline{l}=\left(l_{1}, \ldots, l_{n}\right) \in$ $\operatorname{ker}(A) \subset \mathbb{Z}^{n}$ such that $l_{i}>0$ for $i=1, \ldots, n$. It follows then from assumption (i) that the semi-group $\mathbb{N} A=\sum_{i=1}^{n} \mathbb{N} \underline{a}_{i}$ equals $\mathbb{Z}^{d}$ (since for any $\underline{c} \in \mathbb{Z}^{d}$, a linear combination $\underline{c}=\sum_{i=1}^{n} n_{i} \underline{a}_{i}$ with integer coefficients $n_{i}$ can be turned into a combination with positive coefficents by adding the vector $\underline{0}=\sum_{i=1}^{n} l_{i} \underline{a}_{i}$ sufficiently many times). This fact will be used later (see the proof of Proposition 3.8.

Let $S_{1}=\mathbb{G}_{m}^{d}=\operatorname{Spec}\left(\mathbb{C}\left[y_{1}^{ \pm}, \ldots, y_{d}^{ \pm}\right]\right)$and $S_{2}=\mathbb{G}_{m}^{n}=\operatorname{Spec}\left(\mathbb{C}\left[\lambda_{1}^{ \pm}, \ldots, \lambda_{n}^{ \pm}\right]\right)$be two algebraic tori, and consider the affine space $V=\mathbb{A}^{n+1}$ with coordinates $\lambda_{0}, \lambda_{1}, \ldots, \lambda_{n}$. Let $V^{\vee}$ be the dual space with coordinates $w_{0}, w_{1}, \ldots, w_{n}$, and we also set $\tau:=-w_{0}$ and $z:=\tau^{-1}$. We decompose $V=\mathbb{A}_{\lambda_{0}}^{1} \times W$, and consider $S_{2} \subset W$ as an open subset.

Consider the following family of Laurent polynomials

$$
\begin{aligned}
\varphi: S_{1} \times S_{2} & \longrightarrow \mathbb{A}_{\lambda_{0}}^{1} \times S_{2} \\
(\underline{y}, \underline{\lambda}) & \longmapsto\left(-\sum_{i=1}^{n} \lambda_{i} \underline{y}^{\underline{a}}, \lambda_{1}, \ldots, \lambda_{n}\right),
\end{aligned}
$$

which in the case of the matrix from equation (2) becomes

$$
\varphi\left(y_{1}, \ldots, y_{n-1}, \lambda_{1}, \ldots, \lambda_{n}\right)=\left(-\lambda_{1} \cdot y_{1} \cdot \ldots \cdot y_{n-1}-\frac{\lambda_{2}}{y_{1}}-\ldots-\frac{\lambda_{n}}{y_{n-1}}, \lambda_{1}, \ldots, \lambda_{n}\right) .
$$

Write $f: S_{1} \times S_{2} \rightarrow \mathbb{A}_{\lambda_{0}}^{1}$ for the composition of $\varphi$ with the first projection $\mathbb{A}_{\lambda_{0}}^{1} \times S_{2} \rightarrow \mathbb{A}_{\lambda_{0}}^{1}$. Similarly, for any fixed $\underline{\lambda} \in S_{2}$, we write $f_{\underline{\lambda}}:=f_{\mid S_{1} \times\{\underline{\lambda}\}}: S_{1} \rightarrow \mathbb{A}_{\lambda_{0}}^{1}$ for the restriction of $f$ to the parameter value $\underline{\lambda}$. Let us first quote the following statement from [RS15b, Lemma 2.8.]. Since the input data in loc. cit. are fans of toric varieties, we will copy the proof here to make it fit the assumptions above. Recall (see Kou76]) that a Laurent polynomial $f_{\underline{\lambda}}=\sum_{i=1}^{n} \lambda_{i} \underline{\underline{y}}^{\underline{a_{i}}}$ is called convenient if 0 lies in the interior of $\Delta$ and non-degenerate if for all proper faces $\delta \subset \operatorname{Conv}\left(0, \underline{a}_{1}, \ldots, \underline{a}_{n}\right)$ not containing the origin, the Laurent polynomial $f_{\underline{\lambda}}^{\delta}=\sum_{i: \underline{a}_{i} \in \delta} \lambda_{i} \underline{\underline{y}}^{\underline{a_{i}}}$ has no critical points in $S_{1}$. Notice that for matrices $A$ satisfying assumption (iii) from above, this last condition is equivalent to asking that $f_{\underline{\lambda}}^{\delta}$ is non-singular for all proper faces $\delta \subset \Delta$.

Lemma 3.3. The Laurent polynomial $f_{\underline{\lambda}}: S_{1} \rightarrow \mathbb{A}_{z}^{1}$ is non-degenerate and convenient for any $\underline{\lambda} \in S_{2}$.

Proof. Obviously $f_{\underline{\lambda}}$ is convenient by assumption (iii) from above. Let $\delta \subset \Delta$ be a face of codimension $d+1-l$, with $l=1, \ldots, d$. Let $\left\{i_{1}, \ldots, i_{l}\right\} \subset\{1, \ldots, n\}$ such that $\left\{\underline{a}_{i} \in \delta\right\}=\left\{\underline{a}_{i_{1}}, \ldots, \underline{a}_{i_{l}}\right\}$, notice that because of assumption (ii), we cannot have more than $l$ vectors in a face of dimension $l-1$. Since the vectors $\underline{a}_{i_{1}}, \ldots, \underline{a}_{i_{l}}$ are linearly independent over $\mathbb{Q}$, the matrix

$$
C:=\left(\begin{array}{ccc}
a_{i_{1} 1} & \ldots & a_{i_{l} 1} \\
\vdots & \vdots & \vdots \\
a_{i_{1} d} & \ldots & a_{i_{l} d}
\end{array}\right)
$$

has full rank (equal to $l$ ) and hence the system

$$
C \cdot\left(\begin{array}{c}
\lambda_{i_{1}} \underline{\underline{y}}^{\underline{a}_{i_{1}}} \\
\vdots \\
\lambda_{i_{l}} \underline{\underline{y}}^{\underline{a_{i_{l}}}}
\end{array}\right)=0,
$$

which is the system of critical point equations $\left(y_{k} \partial_{y_{k}} f_{\underline{\lambda}}^{\delta}=0\right)_{k=1, \ldots, d}$, has no nontrivial solution; the trivial one $\lambda_{i} \cdot \underline{y}^{\underline{a}}{ }_{i}=0$ for all $i \in\left\{i_{1}, \ldots, i_{l}\right\}$ is not valid since $\underline{\lambda} \in S_{2}$ and we are looking for solutions $\underline{y} \in S^{1}$. Hence $f_{\underline{\lambda}}^{\delta}$ is non-singular on $S_{1}$, and so $f_{\underline{\lambda}}$ is non-degenerate.

From this we can deduce the following statement, which is a variant of [RS15b, Lem. 2.13]. However, we will give the proof here for the convenience of the reader.

Lemma 3.4. $\widehat{\mathcal{M}}_{A}^{\left(\beta_{0}, \beta\right)}$ is locally $\mathcal{O}_{\mathbb{A}_{z}^{1} \times S_{2}}$-free of rank $n ! \cdot \operatorname{vol}(\Delta)$ (considering the normalized volume in $\mathbb{R}^{n}$ such that $[0,1]^{n}$ has volume one). For the case of the matrix in equation (2), this rank equals $n$. 
Proof. There is an isomorphism of $\mathcal{O}_{\mathrm{A}_{z}^{1} \times S_{2}}$-modules (or even of $\mathcal{R}_{\mathrm{A}_{z}^{1} \times S_{2}}$-modules)

$$
\widehat{\mathcal{M}}_{A}^{\left(\beta_{0}, \beta\right)} \cong \frac{\mathcal{R}_{\mathrm{A}_{z}^{1} \times S_{2}}}{\left(\prod_{j: l_{j}>0}\left(z \partial_{\lambda_{j}}\right)^{l_{j}}-\prod_{j: l_{j}<0}\left(z \partial_{\lambda_{j}}\right)^{-l_{j}}\right)_{l \in \mathbb{L}}+\left(\sum_{j=1}^{n} a_{k j} \lambda_{j} z \partial_{\lambda_{j}}-z \beta_{k}\right)_{k=1, \ldots, d}},
$$

so it suffices to prove the statement for the module on the right hand side of this equation. We consider the filtration induced on it by the filtration on $\mathcal{R}_{\mathbb{A}_{z}^{1} \times S_{2}}$ for which $z \partial_{\lambda_{i}}$ has degree 1 and any element of $\mathcal{O}_{\mathbb{A}_{z}^{1} \times S_{2}}$ has degree zero. The graded module with respect to this filtration is a sheaf on $\mathbb{A}_{z}^{1} \times T^{*} S_{2}$, and we first need to show that its support lies in the zero section, i.e., in the subspace $\mathbb{A}_{z}^{1} \times S_{2}$. Notice that the symbols of the operators in the ideal $\left(\prod_{j: l_{j}>0}\left(z \partial_{\lambda_{j}}\right)^{l_{j}}-\prod_{j: l_{j}<0}\left(z \partial_{\lambda_{j}}\right)^{-l_{j}}\right)_{l \in \mathbb{L}}+$ $\left(\sum_{j=1}^{n} a_{k j} \lambda_{j} z \partial_{\lambda_{j}}-z \beta_{k}\right)_{k=1, \ldots, d}$ with respect to the filtration of $\mathcal{R}_{\mathbb{A}_{z}^{1} \times S_{2}}$ defined above are the same as the symbols of the operators of the usual hypergeometric ideal $\left(\prod_{j: l_{j}>0} \partial_{\lambda_{j}}^{l_{j}}-\prod_{j: l_{j}<0} \partial_{\lambda_{j}}^{-l_{j}}\right)_{l \in \mathbb{L}}+$ $\left(\sum_{j=1}^{n} a_{k j} \lambda_{j} \partial_{\lambda_{j}}-\beta_{k}\right)_{k=1, \ldots, d}$ with respect to the (usual) order filtration on $\mathcal{D}_{S_{2}}$. Hence by the arguments of Ado94, Lem. 3.1 to Lem. 3.3], we obtain that the variety cut out by the symbols of the operators in $\left(\prod_{j: l_{j}>0}\left(z \partial_{\lambda_{j}}\right)^{l_{j}}-\prod_{j: l_{j}<0}\left(z \partial_{\lambda_{j}}\right)^{-l_{j}}\right)_{l \in \mathbb{L}}+\left(\sum_{j=1}^{n} a_{k j} \lambda_{j} z \partial_{\lambda_{j}}-z \beta_{k}\right)_{k=1, \ldots, d}$ is $\mathbb{A}_{z}^{1} \times S_{2}$, notice that here the fact that $f_{\underline{\lambda}}$ is non-degenerate for all $\underline{\lambda} \in S_{2}$ (i.e., the statement of the last lemma) plays a crucial role.

From $\operatorname{supp}\left(\operatorname{gr}\left(\widehat{\mathcal{M}}_{A}^{\left(\beta_{0}, \beta\right)}\right)\right) \subseteq \mathbb{A}_{z}^{1} \times S_{2}$ one deduces as in ordinary $\mathcal{D}$-module theory that $\widehat{\mathcal{M}}_{A}^{\left(\beta_{0}, \beta\right)}$ is $\mathcal{O}_{\mathbb{A}_{z}^{1} \times S_{2}}$-coherent. Now the restriction $\widehat{\mathcal{M}}_{A}^{(0, \beta)} / z \cdot \widehat{\mathcal{M}}_{A}^{(0, \beta)}$ is isomorphic to the Jacobian algebra $\operatorname{Jac}(f)=\mathcal{O}_{S_{1} \times S_{2}} /\left(\partial_{y_{1}} f, \ldots, \partial_{y_{d}} f\right)$ (see RS15b, Lem. 2.12]), and that the latter has rank equal to $n ! \cdot \operatorname{vol}(\Delta)$ (see Kou76, Thm. 1.16]). Moreover, the localized object $\widehat{\mathcal{M}}_{A}^{(0, \beta)} \otimes_{\mathcal{O}_{\mathbb{A}_{z}^{1} \times S_{2}}} \mathcal{O}_{\mathbb{A}_{z}^{1} \times S_{2}}\left[z^{-1}\right]$ has a $\mathcal{D}_{\mathbb{A}_{z}^{1} \times S_{2}}\left[z^{-1}\right]$-module structure, and is $\mathcal{O}_{\mathbb{A}_{z}^{1} \times S_{2}}\left[z^{-1}\right]$-coherent, hence $\mathcal{O}_{\mathbb{A}_{z}^{1} \times S_{2}}\left[z^{-1}\right]$-free. Its rank can also be calculated as the holonomic rank of ordinary GKZ-systems, see RS15b, Prop. 2.7 (3)], and equals $n ! \cdot \operatorname{vol}(\Delta)$.

This shows that the module $\widehat{\mathcal{M}}_{A}^{\left(\beta_{0}, \beta\right)}$ itself is $\mathcal{O}_{\mathrm{A}_{z}^{1} \times S_{2}}$-free of the same rank $n ! \cdot \operatorname{vol}(\Delta)$.

For any complex number $\beta$, recall that the Kummer $\mathcal{D}$-module of parameter $\beta$ is by defintion the quotient $\mathcal{K}_{\beta}:=\mathcal{D}_{\mathbb{G}_{m, t}} /\left(t \partial_{t}-\beta\right)$. We will also use in this section $\mathcal{R}$-modules arising from such $\mathcal{D}$-modules. Here is the precise definition.

Definition 3.5. For any complex number $\beta$, we define the Kummer $\mathcal{R}$-module of parameter $\beta$ as the cyclic $\mathcal{R}_{\mathbb{A}_{z}^{1} \times \mathbb{G}_{m, t}}^{\text {int }}$-module

$$
\widehat{\mathcal{K}}_{\beta}:=\mathcal{R}_{\mathbb{A}^{1} \times \mathbb{G}_{m, t}}^{\text {int }} /\left(z^{2} \partial_{z}, z t \partial_{t}-z \beta\right) .
$$

Remark 3.6. Although both kinds of Kummer modules can be defined for any complex value of their parameters, in the end we will be interested only in the real case to make use of their Hodge properties. Indeed, since both have no singularities at $\mathbb{G}_{m, t}$, if $\mathcal{K}_{\beta}$ were a complex Hodge module it would be in fact a complex variation of Hodge structures, and by (the first part of) the proof of [Sch73, Lem. 4.5], $\beta$ ought to be real.

On the other hand, $\widehat{\mathcal{K}}_{\beta}$ is clearly the Rees module of $\mathcal{K}_{\beta}$ together with the trivial filtration $F_{\bullet}$ such that $F_{k}=0$ for $k<0$ and $F_{k}=\mathcal{K}_{\beta}$ for $k \geq 0$. As described in Moc15a Prop. 13.5.4] and Sab18a, Thm. 0.2], it gives rise to an integrable pure twistor $\mathcal{D}$-module on $\mathbb{G}_{m, t}$, which belongs as well to $\operatorname{IrMHM}\left(\mathbb{G}_{m}\right)$. It is also described as a harmonic bundle at Moc15a, §2.1.9].

Definition 3.7. For any smooth complex algebraic variety $X$, and for any $\beta \in \mathbb{C}^{d}$, we will denote by $\mathcal{O}_{S_{1} \times X}^{\beta}$ the $\mathcal{D}_{S_{1} \times X}$-module corresponding to the structure sheaf of $S_{1} \times X$ twisted by $\underline{y}^{-1-\beta}$, that is,

$$
\mathcal{O}_{S_{1} \times X}^{\beta}:=\frac{\mathcal{D}_{S_{1}}}{\left(y_{k} \partial_{y_{k}}+\beta_{k}+1: k=1, \ldots, d\right)} \otimes \mathcal{O}_{X}=: \mathcal{O}_{S_{1}}^{\beta} \otimes \mathcal{O}_{X}
$$


Note that for any other $\beta^{\prime} \in \mathbb{C}^{d}$ such that $\beta-\beta^{\prime} \in \mathbb{Z}^{d}, \mathcal{O}_{S_{1} \times X}^{\beta} \cong \mathcal{O}_{S_{1} \times X}^{\beta^{\prime}}$. These modules underly complex Hodge modules if and only if the components of the parameter vector are real numbers, since they are the corresponding exterior products of the Kummer modules $\mathcal{K}_{-\beta_{1}}, \ldots, \mathcal{K}_{-\beta_{d}}$ and $\mathcal{O}_{X}$.

With these notations, the following result is a special case of [RS17, Prop. 3.21], taking into account the twist of $\mathcal{O}_{S_{1} \times S_{2}}$ by $y^{-1-\beta}$. However, we prefer to give a direct proof here, which is a simplified variant of the corresponding statement in [Moc15b, Prop. E.6]. Notice that the idea of this approach goes back to the so-called "better behaved GKZ-systems" of Borisov-Horja (see [BH06]).

Proposition 3.8. There exists an isomorphism of $\mathcal{R}_{\mathbb{A}_{z}^{1} \times S_{2}}^{\mathrm{int}}$-modules

$$
\mathcal{H}^{0}\left(\pi_{2, *} \Omega_{S_{1} \times S_{2} / S_{2}}^{\bullet+d}[z], z(d-\kappa(\beta) \wedge)-d f \wedge\right) \longrightarrow \widehat{\mathcal{M}}_{A}^{(0, \beta)}
$$

for any $\beta \in \mathbb{C}^{d}$, given by sending $\omega:=\prod_{j=1}^{d} d y_{j} / y_{j}$ to $[1] \in \widehat{\mathcal{M}}_{A}^{(0, \beta)}$, where we write $\kappa(\beta)$ for $\sum_{j=1}^{d} \beta_{j} d y_{j} / y_{j}$ and $\pi_{2}$ for the the second canonical projection $S_{1} \times S_{2} \rightarrow S_{2}$.

Proof. We will construct a third module $\mathcal{Q} / \mathcal{K}$, and show that both the module $\widehat{\mathcal{M}}_{A}^{(0, \beta)}$ and the module $\mathcal{H}^{0}\left(\pi_{2, *} \Omega_{S_{1} \times S_{2} / S_{2}}^{\bullet+d}[z], z(d-\kappa(\beta) \wedge)-d f \wedge\right)$ are isomorphic to it. For this purpose, define the free $\mathcal{O}_{\mathbb{A}_{z}^{1} \times S_{2}}$-module

$$
\mathcal{Q}:=\bigoplus_{\underline{c} \in \mathbb{Z}^{d}} \mathcal{O}_{\mathrm{A}_{z}^{1} \times S_{2}} \cdot e(\underline{c}),
$$

where $e(\alpha)$ is a symbol representing a generator of $\mathcal{Q}$. We put a $\mathcal{R}_{\mathbb{A}_{z}^{1} \times S_{2}}^{\text {int }}$-module structure on $\mathcal{Q}$ by letting

$$
\begin{aligned}
z \partial_{\lambda_{i}} e(\underline{c}) & :=e\left(\underline{c}+\underline{a}_{i}\right), \\
z^{2} \partial_{z} e(\underline{c}) & :=-\sum_{i=1}^{n} \lambda_{i} \cdot z \partial_{\lambda_{i}} e(\underline{c}) .
\end{aligned}
$$

Since we have $\mathbb{N} A=\mathbb{Z}^{d}$ (see Remark 3.2 , (ii)), we conclude from the first of these equations that $\mathcal{Q}$ is a cyclic $\mathcal{R}_{\mathbb{A} 1}^{\mathrm{int} \times S_{2}}$-module with generator $e(\underline{0})$. Moreover, we consider the $\mathcal{R}_{\mathbb{A}_{z}^{1} \times S_{2}}^{\text {int }}$-submodule $\mathcal{K}$ of $\mathcal{Q}$ generated by

$$
\left(\sum_{i=1}^{n} a_{k i} \lambda_{i} \cdot z \partial_{\lambda_{i}}+z\left(c_{k}-\beta_{k}\right)\right) \cdot e(\underline{c}) \quad \forall \underline{c} \in \mathbb{Z}^{d}, \forall k=1, \ldots, d .
$$

Then we claim that there is an $\mathcal{R}_{\mathrm{A}_{z}^{1} \times S_{2}}^{\text {int }}$-isomorphism

$$
\phi: \widehat{\mathcal{M}}_{A}^{(0, \beta)} \stackrel{\cong}{\longrightarrow} / \mathcal{K} \text {. }
$$

sending [1] to $e(\underline{0})$. It is clear that $\phi$ is well defined since all operators occurring in the denominator in the definition of $\widehat{\mathcal{M}}_{A}^{(0, \beta)}$ act by zero in $\mathcal{Q} / \mathcal{K}$ : The operators $\prod_{j: l_{j}>0}\left(z \partial_{\lambda_{j}}\right)^{l_{j}}-\prod_{j: l_{j}<0}\left(z \partial_{\lambda_{j}}\right)^{-l_{j}}$ act by zero already on $\mathcal{Q}$ (as $\underline{l} \in \mathbb{L}$ ); similarly, $z^{2} \partial_{z}+\lambda_{1} z \partial_{\lambda_{1}}+\ldots+\lambda_{n} z \partial_{\lambda_{n}}$ acts by zero on $\mathcal{Q}$ because of the definition of the action of $z^{2} \partial_{z}$, and $\sum_{j=1}^{n} a_{k j} \lambda_{j} z \partial_{\lambda_{j}}-z \beta_{k}, k=1, \ldots, d$ is a generator of $\mathcal{K}$, so its class is obviously zero in the quotient $\mathcal{Q} / \mathcal{K}$.

An inverse map $\mathcal{Q} / \mathcal{K} \rightarrow \widehat{\mathcal{M}}_{A}^{(0, \beta)}$ is defined as follows: For any $\underline{c} \in \mathbb{N}^{d}$, choose a representation $\underline{c}=\sum_{i=1}^{n} n_{i} \underline{a}_{i}$, with $n_{i} \in \mathbb{N}$. Then we map $e(\underline{c})$ to the class of $\prod_{i=1}^{n}\left(z \partial_{\lambda_{i}}\right)^{n_{i}}$ in $\widehat{\mathcal{M}}_{A}^{(0, \beta)}$. This is well defined: If we take another representation $\underline{c}=\sum_{i=1}^{n} n_{i}^{\prime} \underline{a}_{i}$, then $\left(n_{i}-n_{i}^{\prime}\right)_{i=1, \ldots, n} \in \mathbb{Z}^{n}$ lies in $\mathbb{L}$, showing that

from what it follows that

$$
\prod_{i: n_{i}>n_{i}^{\prime}}\left(z \partial_{\lambda_{i}}\right)^{n_{i}-n_{i}^{\prime}}-\prod_{i: n_{i}^{\prime}>n_{i}}\left(z \partial_{\lambda_{i}}\right)^{n_{i}^{\prime}-n_{i}}=0 \in \widehat{\mathcal{M}}_{A}^{(0, \beta)}
$$

$$
\prod_{i=1}^{n}\left(z \partial_{\lambda_{i}}\right)^{n_{i}}=\prod_{i=1}^{n}\left(z \partial_{\lambda_{i}}\right)^{n_{i}^{\prime}} \in \widehat{\mathcal{M}}_{A}^{(0, \beta)} .
$$

Moreover, elements of $\mathcal{K}$ obviously go to zero in $\widehat{\mathcal{M}}_{A}^{(0, \beta)}$. It is also clear that this map is inverse to $\phi$. 
In order to identify $\mathcal{Q} / \mathcal{K}$ with the twisted de Rham cohomology module, notice that the complex $\left(\pi_{2, *} \Omega_{S_{1} \times S_{2} / S_{2}}^{\bullet+d}[z], z(d-\kappa(\beta) \wedge)-d f \wedge\right)$ is concentrated in degrees $-d$ to 0 , so we need to compute its top cohomology, i.e., the quotient

$$
\frac{\pi_{2, *} \Omega_{S_{1} \times S_{2} / S_{2}}^{d}[z]}{(z(d-\kappa(\beta) \wedge)-d f \wedge) \pi_{2, *} \Omega_{S_{1} \times S_{2} / S_{2}}^{d-1}[z]}
$$

Since we have $\mathbb{C}\left[\mathbb{Z}^{d}\right] \cong H^{0}\left(S_{1}, \mathcal{O}_{S_{1}}\right)$, there is an obvious identification of $\mathcal{O}_{\mathbb{A}_{z}^{1} \times S_{2}}$-modules,

$$
\mathcal{Q} \cong \pi_{2, *} \Omega_{S_{1} \times S_{2} / S_{2}}^{d}[z]
$$

sending $e(\underline{c})$ to $\underline{y}^{\underline{c}} \cdot \omega$. Now it is easy to see (cf. the proofs of Moc15b, Prop. E.4 and E.6]) that the image of

$$
(z(d-\kappa(\beta) \wedge)-d f \wedge) \pi_{2, *} \Omega_{S_{1} \times S_{2} / S_{2}}^{d-1}[z]
$$

can be identified under this isomorphism with the submodule $\mathcal{K}$ of $\mathcal{Q}$. Moreover, one checks that the $\mathcal{R}_{\mathbb{A}_{z}^{1} \times S_{2}}^{\text {int }}$-module structures on the quotient (5) and the module $\mathcal{Q} / \mathcal{K}$ are compatible, e.g., we have $z \partial_{\lambda_{i}} \omega=-\left(\partial_{\lambda_{i}} f\right) \omega=\underline{y}^{\underline{a}} \omega$, accordingly with $\left(z \partial_{\lambda_{i}}\right) \cdot e(\underline{0})=e\left(\underline{a}_{i}\right)$.

Combining the two isomorphisms $\mathcal{Q} / \mathcal{K} \cong \mathcal{H}^{0}\left(\pi_{2, *} \Omega_{S_{1} \times S_{2} / S_{2}}^{\bullet+d}[z], z(d-\kappa(\beta) \wedge)-d f \wedge\right)$ and $\widehat{\mathcal{M}}_{A}^{(0, \beta)} \cong$ $\mathcal{Q} / \mathcal{K}$ we obtain the desired one

$$
\mathcal{H}^{0}\left(\pi_{2, *} \Omega_{S_{1} \times S_{2} / S_{2}}^{\bullet+d}[z], z(d-\kappa(\beta) \wedge)-d f \wedge\right) \longrightarrow \widehat{\mathcal{M}}_{A}^{(0, \beta)}
$$

which sends $\omega$ to [1].

In the sequel, we need to consider an extension of the map $\varphi: S_{1} \times S_{2} \rightarrow \mathbb{A}_{\lambda_{0}}^{1} \times S_{2}$ to a projective morphism. For that purpose, we will construct a partial compactification of $S_{1} \times S_{2}$ by looking at a toric compactification of $S_{1}$. More precisely, consider the embedding

$$
\begin{aligned}
g: S_{1} & \hookrightarrow \mathbb{P}\left(V^{\vee}\right)=\mathbb{P}^{n} \\
\underline{y} & \longmapsto\left(w_{0}: \ldots: w_{n}\right)=\left(1: \underline{y}^{\underline{a}_{1}}: \ldots: \underline{y}^{\underline{a_{n}}}\right)
\end{aligned}
$$

and put $X:=\overline{\operatorname{Im}(g)}$. Consider the graph $\Gamma_{f} \subset S_{1} \times \mathbb{A}_{\lambda_{0}}^{1} \times S_{2}$, and let $\Gamma X$ be the closure of $\Gamma_{f}$ in $X \times \mathbb{A}^{1} \times S_{2}$. Then we have $\Gamma X \subset Z^{*}$, where

$$
Z^{*}:=\left\{\sum_{i=0}^{n} \lambda_{i} \cdot w_{i}=0\right\} \stackrel{i_{P}}{\longleftrightarrow} \mathbb{P}^{n} \times \mathbb{A}_{\lambda_{0}}^{1} \times S_{2}=: P
$$

is the universal hyperplane. We thus have the commutative diagram

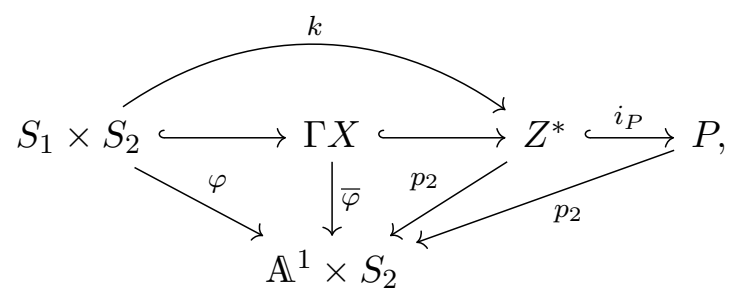

where the map $S_{1} \times S_{2} \hookrightarrow \Gamma X$ is the composition of the isomorphism $S_{1} \times S_{2} \cong \Gamma_{f}$ with $\Gamma_{f} \hookrightarrow \Gamma X$ and the map $\Gamma X \hookrightarrow Z^{*}$ is the closed embedding mentioned above. Notice that $\bar{\varphi}$ is projective, and restricts to $\varphi$ on $S_{1} \times S_{2}$, i.e., it is the extension of $\varphi$ we were looking for.

We need also the following important geometric property of the morphisms $\varphi$ and $\bar{\varphi}$. Recall (see Sab06, §8]) that for a smooth affine variety $U$, a regular function $h: U \rightarrow \mathbb{A}^{1}$ with isolated critical points is called cohomologically tame if there is a partial compactification $j: U \hookrightarrow \bar{U}$ (i.e. $\bar{U}$ is quasiprojective) and an extension of $h$ to a projective morphism $\bar{h}: \bar{U} \rightarrow \mathbb{A}^{1}$ such that the sheaf $\mathbf{R} j_{*} \mathbb{Q}_{U}$ has no vanishing cycles with respect to $\bar{h}$ outside of $U$, i.e., such that for any $c \in \mathbb{A}^{1}$, we have

$$
\operatorname{supp}\left(\psi_{\bar{h}-c}\left(\mathbf{R} j_{*} \mathbb{Q}_{U}\right)\right) \subset U .
$$


We call a morphism $H: \mathcal{X} \rightarrow \mathcal{S}$ on a quasi-projective variety $\mathcal{X}$ stratified smooth if there is a locally finite stratification by locally closed smooth subvarieties such that the restriction of $H$ to any strata has no critical points.

Lemma 3.9. We have the following properties of the partial compactifications defined above:

(1) For any parameter value $\underline{\lambda} \in S_{2}$, the morphism $f_{\underline{\lambda}}: S_{1} \rightarrow \mathbb{A}_{\lambda_{0}}^{1}$ is cohomologically tame.

(2) The morphism $\bar{\varphi}: \Gamma X \rightarrow \mathbb{A}^{1} \times S_{2}$ is stratified smooth on its boundary $\Gamma X \backslash\left(S_{1} \times S_{2}\right)$.

(3) Consider the composition $k^{\prime}: S_{1} \times S_{2} \hookrightarrow P$ of the map $k: S_{1} \times S_{2} \hookrightarrow Z^{*}$ with the canonical closed embedding $i_{P}: Z^{*} \hookrightarrow P$. Then for any $\beta \in \mathbb{C}^{d}$ and for $\star \in\{+, \dagger\}$, the module $\left(k^{\prime}\right)_{\star} \mathcal{O}_{S_{1} \times S_{2}}^{\beta}$ is non-characteristic with respect to $p_{2}$ along the boundary $\overline{\operatorname{im}\left(k^{\prime}\right)} \backslash \operatorname{im}\left(k^{\prime}\right)$, i.e., its characteristic variety satisfies $\operatorname{char}\left(k_{\star} \mathcal{O}_{S_{1} \times S_{2}}^{\beta}\right) \cap\left(\mathbb{P}^{n} \times T^{*}\left(\mathbb{A}_{\lambda_{0}}^{1} \times S_{2}\right)\right)_{\mid T^{*}\left(P \backslash \operatorname{im}\left(k^{\prime}\right)\right)} \subset P \backslash \operatorname{im}\left(k^{\prime}\right)$.

Proof. (1) According to Lemma 3.3, $f_{\underline{\lambda}}$ is non-degenerate and convenient for any $\underline{\lambda} \in S_{2}$. It then follows from DL91, Lem. 3.4] that $f_{\underline{\lambda}}$ is cohomologically tame, but since the notations in loc. cit. differ considerably from our situation, we recall the proof: We will show that the extension $\bar{f}_{\underline{\lambda}}: \Gamma X^{\underline{\lambda}}:=(\Gamma X) \cap\left(\mathbb{P}^{n} \times \mathbb{A}_{\lambda_{0}}^{1} \times\{\underline{\lambda}\}\right) \rightarrow \mathbb{A}_{\lambda_{0}}^{1}$ which is the restriction of $\bar{\varphi}$ over $\mathbb{A}_{\lambda_{0}}^{1} \times\{\underline{\lambda}\}$ has no vanishing cycles at infinity. Recall that the projective toric variety $X$ is stratified by $X=\bigcup_{\delta \subset \Delta} X_{\delta}$, where $\Delta$ is the convex hull in $\mathbb{R}^{d}$ of the columns of the matrix $A, \delta$ is a face of $\Delta$, and $X_{\delta}$ is a torus orbit associated with the face $\delta$ (cf., for instance, [GKZ94, Prop. 5.1.9]). We obtain an induced stratification $\left(\Gamma X_{\delta}\right)_{\delta \in \Delta}$ of $\Gamma X$ and an induced stratification $\left(\Gamma X_{\delta}^{\lambda}\right)_{\delta \in \Delta}$ of the restriction $\Gamma X^{\underline{\lambda}}$. Then the smoothness of the restriction $\left(\bar{f}_{\underline{\lambda}}\right)_{\mid \Gamma X_{\bar{\delta}}^{\lambda}}$ follows from the smoothness of the Laurent polynomial $f_{\underline{\lambda}}^{\delta}=\sum_{i: \underline{a}_{i} \in \delta} \lambda_{i} \underline{y}^{\underline{a}}$, which has no critical points since $f_{\underline{\lambda}}$ is nondegenerate (see Lemma 3.3). Hence we see that $\bar{f}_{\underline{\lambda}}$ is stratified smooth on its boundary, i.e., outside $\Gamma X_{\bar{\Delta}}^{\underline{\lambda}}=\left(\Gamma X \backslash \Gamma_{f}\right) \cap\left(\mathbb{P}^{n} \times \mathbb{A}_{\lambda_{0}}^{1} \times\{\underline{\lambda}\}\right) \cong S_{1}$. Then it follows from Dim04, Prop. 4.2.8] that for any constructible complex $\mathcal{F}^{\bullet}$ on $\Gamma X^{\underline{\lambda}}$ (with respect to the stratification $\left(\Gamma X_{\delta}^{\lambda}\right)_{\delta \subset \Delta}$ ) we have $\psi_{\bar{f}_{\underline{\lambda}}-c} \mathcal{F}_{\mid\left(\Gamma X \underline{\lambda} \backslash \Gamma X_{\bar{\Delta}}\right)}^{\bullet}=0$ for any $c \in \mathbb{A}_{\lambda_{0}}^{1}$. Hence $\operatorname{supp}\left(\psi_{\bar{f}_{\underline{\lambda}}-c} \mathcal{F}^{\bullet}\right) \subset \Gamma X_{\Delta}^{\underline{\lambda}} \cong S_{1}$, and so $f_{\underline{\lambda}}$ is cohomologically tame since we can apply this to the case where $\mathcal{F}^{\bullet}=\mathbf{R} j_{*} \mathbb{Q}_{S_{1}}$, $j: S_{1} \cong \Gamma X_{\bar{\Delta}}^{\lambda} \hookrightarrow \Gamma X^{\underline{\lambda}}$ being the canonical open embedding.

(2) The same proof as in (1) applies, i.e., the restriction $\bar{\varphi}_{\mid \Gamma X_{\delta}}: \Gamma X_{\delta} \rightarrow \mathbb{A}_{\lambda_{0}}^{1} \times S_{2}$ is non-singular for all $\delta \subsetneq \Delta$.

(3) We have $\operatorname{char}\left(\left(k^{\prime}\right)_{\star} \mathcal{O}_{S_{1} \times S_{2}}^{\beta}\right) \subset \bigcup_{\delta \in \Delta} T_{\Gamma X_{\delta}}^{*} P$. The fact that $\bar{\varphi}_{\mid \Gamma X_{\delta}}$ is smooth (for $\delta \subsetneq \Delta$ ) means exactly that the fibres of $p_{2}: P \rightarrow \mathbb{A}_{\lambda_{0}}^{1} \times S_{2}$ are transversal to $\Gamma X_{\delta}$. As the conormal bundle to these fibres is precisely the space $\mathbb{P}^{n} \times T^{*}\left(\mathbb{A}_{\lambda_{0}}^{1} \times S_{2}\right)$, we obtain $\left(\mathbb{P}^{n} \times T^{*}\left(\mathbb{A}_{\lambda_{0}}^{1} \times S_{2}\right)\right) \cap \Gamma X_{\delta} \subset$ $\mathbb{P}^{n} \times \mathbb{A}_{\lambda_{0}}^{1} \times S_{2}$, for all $\delta \subsetneq \Delta$, and so we have $\operatorname{char}\left(k_{\star} \mathcal{O}_{S_{1} \times S_{2}}^{\beta}\right) \cap\left(\mathbb{P}^{n} \times T^{*}\left(\mathbb{A}_{\lambda_{0}}^{1} \times S_{2}\right)\right)_{\mid T^{*}\left(P \backslash i m\left(k^{\prime}\right)\right)} \subset$ $P \backslash \operatorname{im}\left(k^{\prime}\right)$, as required.

In order to achieve the comparison results of this section we need to use several variants of the Fourier-Laplace transformation; let us recall here the definitions.

Definition 3.10. Let $Y$ be a smooth algebraic variety, $U$ be a finite-dimensional complex vector space and $U^{\prime}$ its dual vector space. Denote by $\mathcal{E}$ the trivial vector bundle $\tau: U \times Y \rightarrow Y$ and by $\mathcal{E}^{\prime}$ its dual. Write can : $U \times U^{\prime} \rightarrow \mathbb{A}^{1}$ for the canonical morphism defined by $\operatorname{can}(a, \varphi)=\varphi(a)$. This extends to a function can : $\mathcal{E} \times_{Y} \mathcal{E}^{\prime} \rightarrow \mathbb{A}^{1}$. Define $\mathcal{L}:=\mathcal{O}_{\mathcal{E} \times_{Y} \mathcal{E}^{\prime}} \cdot e^{- \text {can }}$, the free rank one module with differential given by the product rule. Consider also the canonical projections $p_{1}: \mathcal{E} \times_{Y} \mathcal{E}^{\prime} \rightarrow \mathcal{E}, p_{2}: \mathcal{E} \times_{Y} \mathcal{E}^{\prime} \rightarrow \mathcal{E}^{\prime}$. The partial Fourier-Laplace transformation is then defined by

$$
\mathrm{FL}_{Y}(\bullet):=p_{2,+}\left(p_{1}^{+}(\bullet) \otimes_{\mathcal{O}_{\mathcal{E} \times \mathcal{Y}^{\prime}}} \mathcal{L}\right) .
$$

If the base $Y$ is a point we recover the usual Fourier-Laplace transformation and we will simply write FL. Notice that although this functor is defined at the level of derived categories, it is $t$-exact in the derived category of bounded complexes of $\mathcal{D}$-modules with holonomic cohomologies, i.e., induces a functor $\mathrm{FL}_{Y}: \operatorname{Mod}_{\mathrm{h}}\left(\mathcal{D}_{\mathcal{E}}\right) \rightarrow \operatorname{Mod}_{\mathrm{h}}\left(\mathcal{D}_{\mathcal{E}^{\prime}}\right)$. 
We also need the following variant of the Fourier-Laplace transformation.

Definition 3.11. Keep the notations of the previous definition, and assume moreover that $U$ and $U^{\prime}$ are one-dimensional, with respective coordinates $t$ and $\tau$. Put $z=\tau^{-1}$, and denote by $j_{\tau}: \mathbb{G}_{m, \tau} \hookrightarrow \mathbb{A}_{\tau}^{1}$ and $j_{z}: \mathbb{G}_{m, \tau} \hookrightarrow \mathbb{A}_{z}^{1}=\mathbb{P}_{\tau}^{1} \backslash\{\tau=0\}$ the canonical embeddings. Then the localized partial FourierLaplace transformation with respect to $\tau$ is defined by

$$
\mathrm{FL}_{Y}^{\text {loc }}:=\left(j_{z} \times \operatorname{id}_{Y}\right)_{+}\left(j_{\tau} \times \operatorname{id}_{Y}\right)^{+} \mathrm{FL}_{Y} .
$$

Our next aim is to compare the twisted de Rham cohomology of the family $\varphi: S_{1} \times S_{2} \rightarrow \mathbb{A}_{\lambda_{0}}^{1} \times S_{2}$, i.e., the left-hand side of the isomorphism of Proposition 3.8 with an object derived from a certain intersection cohomology $\mathcal{D}$-module on $Z^{*}$. Recall that for a smooth algebraic variety $Y$, and an open subvariety $j: U \hookrightarrow Y$, we call intersection cohomology module with coefficients in some $\mathcal{D}_{U}$-module $\mathcal{N}$ the intermediate extension $j_{\dagger+} \mathcal{N}:=\operatorname{im}\left(j_{\dagger} \mathcal{N} \rightarrow j_{+} \mathcal{N}\right)$. Its name comes from the fact that, if $\mathcal{N}$ is smooth and corresponds to a local system $\mathcal{L}$ on $U$ under the Rieman-Hilbert correspondence, $j_{\dagger+} \mathcal{N}$ corresponds to the intersection cohomology complex on $Y$ with coefficients in $\mathcal{L}, I C(Y, \mathcal{L})$.

Now is when we can properly state and prove the next comparison result of this section. Recall that we take a matrix $A \in \mathrm{M}(d \times n, \mathbb{Z})$ satisfying the assumptions 3.1 and a parameter vector $\beta \in \mathbb{C}^{d}$. Recall that $Z^{*}=\left\{\sum_{i=0}^{n} w_{i} \lambda_{i}=0\right\} \subset P=\mathbb{P}^{n} \times \mathbb{A}_{\lambda_{0}}^{1} \times S_{2}$ denotes the universal hyperplane, the map $k$, introduced in diagram (7), is

$$
\begin{aligned}
k: S_{1} \times S_{2} & \longrightarrow Z^{*} \\
(\underline{y}, \underline{\lambda}) & \longmapsto\left(\left(1: \underline{y}^{\underline{a_{1}}}: \ldots: \underline{y}^{\underline{a}_{n}}\right),\left(-\sum_{i=1}^{n} \lambda_{i} \underline{y}^{\underline{a}_{1}}, \lambda_{1}, \ldots, \lambda_{n}\right)\right)
\end{aligned}
$$

and $p_{2}$ is the restriction of the canonical projection from $P$ to $\mathbb{A}^{1} \times S_{2}$ to the subspace $Z^{*}$.

Proposition 3.12. In the above situation, we have the following isomorphism of $\mathcal{D}_{\mathrm{A}_{z}^{1} \times S_{2}}$-modules:

$$
\mathrm{FL}_{S_{2}}^{\text {loc }} \mathcal{H}^{0} p_{2,+} k_{\dagger+} \mathcal{O}_{S_{1} \times S_{2}}^{\beta} \stackrel{\sim}{\longrightarrow} \mathrm{FL}_{S_{2}}^{\text {loc }} \mathcal{H}^{0} \varphi_{+} \mathcal{O}_{S_{1} \times S_{2}}^{\beta} .
$$

Moreover, this isomorphism is induced from the canonical morphism $k_{\dagger+} \mathcal{O}_{S_{1} \times S_{2}}^{\beta} \hookrightarrow k_{+} \mathcal{O}_{S_{1} \times S_{2}}^{\beta}$ by applying the functor $\mathrm{FL}_{S_{2}}^{\text {loc }} \mathcal{H}^{0} p_{2,+}$.

Before entering into the proof of this Proposition, we will need to state some facts about Radon transformations for $\mathcal{D}$-modules. We follow [Rei14, $\S 2]$. Recall that $V$ and $V^{\vee}$ are dual affine spaces of dimension $n+1$ with coordinates $w_{0}, \ldots, w_{n}$ and $\lambda_{0}, \ldots, \lambda_{n}$ respectively.

Definition 3.13. Denote by $Z \subset \mathbb{P}\left(V^{\vee}\right) \times V$ the universal hyperplane with equation $\sum_{i=0}^{n} w_{i} \lambda_{i}=0$ and by $U:=\left(\mathbb{P}\left(V^{\vee}\right) \times V\right) \backslash Z$ its complement. Consider the following commutative diagram:

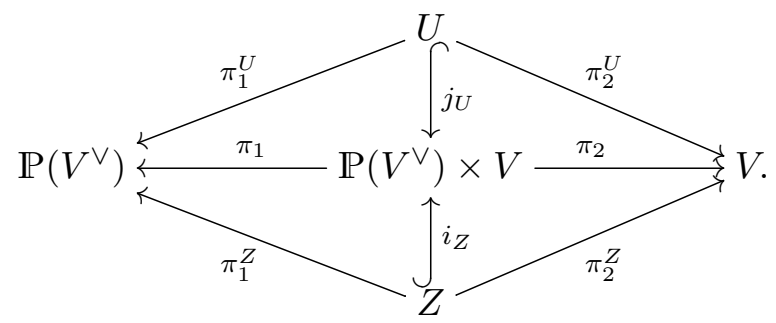

The Radon transformations are functors from $\mathrm{D}^{\mathrm{b}}\left(\mathcal{D}_{\mathbb{P}\left(V^{\vee}\right)}\right)$ to $\mathrm{D}^{\mathrm{b}}\left(\mathcal{D}_{V}\right)$ given by

$$
\begin{aligned}
\mathcal{R} & :=\pi_{2,+}^{Z} \pi_{1}^{Z,+} \cong \pi_{2,+} i_{Z,+} i_{Z}^{+} \pi_{1}^{+}, \\
\mathcal{R}^{\circ} & :=\pi_{2,+}^{U} \pi_{1}^{U,+} \cong \pi_{2,+} j_{U,+} j_{U}^{+} \pi_{1}^{+}, \\
\mathcal{R}_{c}^{\circ} & :=\pi_{2, \dagger}^{U} \pi_{1}^{U,+} \cong \pi_{2,+} j_{U, \dagger} j_{U}^{+} \pi_{1}^{+}, \\
\mathcal{R}_{c s t} & :=\pi_{2,+} \pi_{1}^{+} .
\end{aligned}
$$

Proposition 3.14. Let $g$ be as in (6). Then, for every $\beta \in \mathbb{C}^{d}$, we have the following two exact sequences of regular holonomic $\mathcal{D}_{V}$-modules, which are dual to each other:

$$
0 \longrightarrow \mathcal{H}^{-1} \mathcal{R}_{c s t} g_{+} \mathcal{O}_{S_{1}}^{\beta} \longrightarrow \mathcal{H}^{0} \mathcal{R} g_{+} \mathcal{O}_{S_{1}}^{\beta} \longrightarrow \mathcal{H}^{0} \mathcal{R}_{c}^{\circ} g_{+} \mathcal{O}_{S_{1}}^{\beta} \longrightarrow \mathcal{H}^{0} \mathcal{R}_{c s t} g_{+} \mathcal{O}_{S_{1}}^{\beta} \longrightarrow 0
$$




$$
0 \longrightarrow \mathcal{H}^{0} \mathcal{R}_{c s t} g_{\dagger} \mathcal{O}_{S_{1}}^{-\beta} \longrightarrow \mathcal{H}^{0} \mathcal{R}^{\circ} g_{\dagger} \mathcal{O}_{S_{1}}^{-\beta} \longrightarrow \mathcal{H}^{0} \mathcal{R} g_{\dagger} \mathcal{O}_{S_{1}}^{-\beta} \longrightarrow \mathcal{H}^{1} \mathcal{R}_{c s t} g_{\dagger} \mathcal{O}_{S_{1}}^{-\beta} \longrightarrow 0
$$

Moreover, the $\mathcal{D}_{V}$-modules $\mathcal{H}^{i} \mathcal{R}_{c s t} g_{\star} \mathcal{O}_{S_{1}}^{\beta}$, for $i \in\{-1,0,1\}$ and $\star \in\{+, \dagger\}$, that appear in the above sequences are $\mathcal{O}_{V}$-free. Consequently, for any $\beta \in \mathbb{C}^{d}$, calling $j_{V^{*}}$ the canonical inclusion $V^{*}:=$ $\mathbb{A}_{\lambda_{0}}^{1} \times S_{2} \hookrightarrow V$, we have isomorphisms of $\mathcal{D}_{\mathbb{A}_{z}^{1} \times S_{2}}$-modules

$$
\begin{aligned}
\mathrm{FL}_{S_{2}}^{\text {loc }} j_{V^{*}}^{+} \mathcal{R}_{c}^{\circ} g_{+} \mathcal{O}_{S_{1}}^{\beta} \cong \mathrm{FL}_{S_{2}}^{\text {loc }} j_{V^{*}}^{+} \mathcal{R} g_{+} \mathcal{O}_{S_{1}}^{\beta}, \\
\mathrm{FL}_{S_{2}}^{\text {loc }} j_{V^{*}}^{+} \mathcal{R}^{\circ} g_{\dagger} \mathcal{O}_{S_{1}}^{\beta} \cong \mathrm{FL}_{S_{2}}^{\text {loc }} j_{V^{*}}^{+} \mathcal{R} g_{\dagger} \mathcal{O}_{S_{1}}^{\beta}
\end{aligned}
$$

Proof. Following the notation of the previous definition, since $Z$ is smooth, the excision triangle ([HTT08, Prop. 1.7.1]) corresponding to the diagram $U \hookrightarrow \mathbb{P}\left(V^{\vee}\right) \times V \leftarrow Z$ gives rise to the following triangles of Radon transformations for any $\mathcal{M} \in \mathrm{D}_{\mathrm{c}}^{\mathrm{b}}\left(\mathcal{D}_{\mathbb{P}\left(V^{\vee}\right)}\right)$ :

$$
\begin{aligned}
& \mathcal{R}_{c s t} \mathcal{M} \longrightarrow \mathcal{R}^{\circ} \mathcal{M} \longrightarrow \mathcal{R} \mathcal{M} \longrightarrow, \\
& \mathcal{R} \mathcal{M} \longrightarrow \mathcal{R}_{c}^{\circ} \mathcal{M} \longrightarrow \mathcal{R}_{c s t} \mathcal{M} \longrightarrow,
\end{aligned}
$$

where the second triangle is dual to the first.

Note that $\mathbb{D O}_{S_{1}}^{\beta} \cong \mathcal{O}_{S_{1}}^{-1-\beta} \cong \mathcal{O}_{S_{1}}^{-\beta}$. It suffices then to show the existence of the first exact sequence of the statement of the proposition and the fact that the $\mathcal{H}^{i} \mathcal{R}_{c s t} g_{\star} \mathcal{O}_{S_{1}}^{\beta}$ are constant. The existence of the exact sequences here follows by just a variation of [Rei14, Prop. 2.8], but considering the twist by $y^{-1-\beta}$ of $\mathcal{O}_{S_{1}}$. That needs in turn [ibid., Prop. 2.5, 2.7, Lem. 2.6], but almost every argument is functorial and anyway they can be easily adapted to our context. The constancy of $\mathcal{H}^{i} \mathcal{R}_{c s t} g_{\star} \mathcal{O}_{S_{1}}^{\beta}$ can be proved as the second point of [ibid., Lem. 2.7].

Proof of Proposition 3.12. Call $p_{1}$ the restriction to $Z^{*}$ of the projection $\mathbb{P}^{n} \times \mathbb{A}_{\lambda_{0}}^{1} \times S_{2} \rightarrow \mathbb{P}^{n}$, abusing a bit of the notation. Consider the cartesian diagram

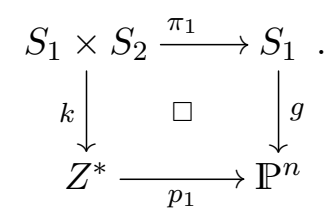

By base change, we have that

$$
k_{+} \mathcal{O}_{S_{1} \times S_{2}}^{\beta} \cong p_{1}^{+} g_{+} \mathcal{O}_{S_{1}}^{\beta}
$$

for any $\beta \in \mathbb{C}^{d}$. Now, since $p_{1}$ is smooth, we have the analogous isomorphism $k_{\dagger} \mathcal{O}_{S_{1} \times S_{2}}^{\beta} \cong p_{1}^{+} g_{\dagger} \mathcal{O}_{S_{1}}^{\beta}$ for every $\beta$ as well. Now note that because of $p_{1}$ being non-characteristic, it is easy to show (cf. the proof of the third point of RS17, Prop. 2.22]) that for every $\beta$

$$
k_{\dagger+} \mathcal{O}_{S_{1} \times S_{2}}^{\beta} \cong p_{1}^{+} g_{\dagger+} \mathcal{O}_{S_{1}}^{\beta} .
$$

Consider now the commutative diagram

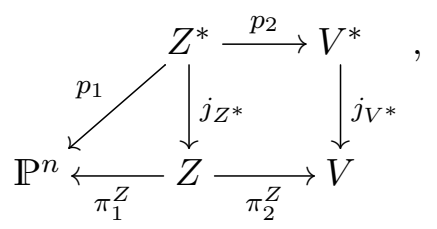

where the square is also cartesian. By applying base change again, we obtain the natural transformations of functors from $\mathrm{D}_{\mathrm{c}}^{\mathrm{b}}\left(\mathcal{D}_{\mathbb{P}^{n}}\right)$ to $\mathrm{D}_{\mathrm{c}}^{\mathrm{b}}\left(\mathcal{D}_{V^{*}}\right)$

$$
p_{2,+} p_{1}^{+} \cong p_{2,+} j_{Z^{*}}^{+} \pi_{1}^{Z,+} \cong j_{V^{*}}^{+} \pi_{2,+}^{Z} \pi_{1}^{Z,+}=j_{V^{*}}^{+} \mathcal{R} .
$$

Therefore, applying $p_{2,+}$ to isomorphisms (10) and (11) (and taking into account that $\varphi=p_{2} \circ k$, see diagram (7)) we obtain

$$
\varphi_{+} \mathcal{O}_{S_{1} \times S_{2}}^{\beta} \cong p_{2,+} k_{+} \mathcal{O}_{S_{1} \times S_{2}}^{\beta} \cong j_{V^{*}}^{+} \mathcal{R} g_{+} \mathcal{O}_{S_{1}}^{\beta}, \quad \text { and } \quad p_{2,+} k_{\dagger+} \mathcal{O}_{S_{1} \times S_{2}}^{\beta} \cong j_{V^{*}}^{+} \mathcal{R} g_{\dagger+} \mathcal{O}_{S_{1}}^{\beta} \text {, }
$$

for any $\beta \in \mathbb{C}^{d}$. 
We need now to relate the various Radon transformations with the Fourier-Laplace transformation FL $: \operatorname{Mod}_{\mathrm{h}}\left(\mathcal{D}_{V^{v}}\right) \rightarrow \operatorname{Mod}_{\mathrm{h}}\left(\mathcal{D}_{V}\right)$. This is possible due to the fundamental result of d'Agnolo and Eastwood [DE03, Prop. 1]. We quote the formulation from the proof of [RS17, Lem. 2.12]. Let $B l_{0}\left(V^{\vee}\right) \subset \mathbb{P}^{n} \times V^{\vee}$ be the blow-up of $V^{\vee}$ at the origin and consider the commutative diagram

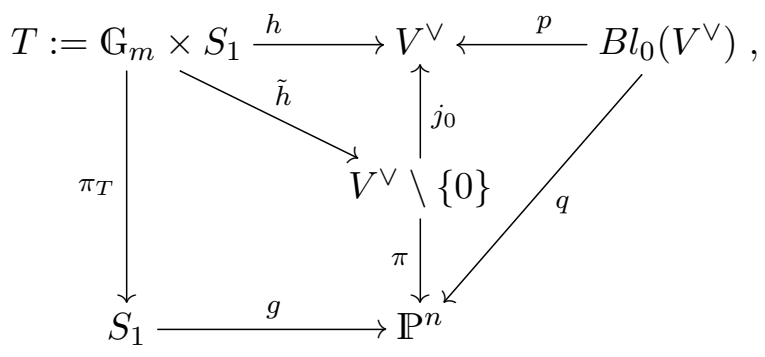

where $\pi$ is the canonical morphism $V^{\vee} \backslash\{0\} \rightarrow \mathbb{P}\left(V^{\vee}\right), \pi_{T}$ is the second projection, and $h$ and $\tilde{h}$ are given by $\left(y_{0}, \underline{y}\right) \mapsto\left(y_{0}, y_{0} \underline{y}^{\underline{a}_{1}}, \ldots, y_{0} \underline{\underline{a}}^{\underline{a_{d}}}\right)$. Then we have the natural transformations

$$
\mathcal{R}_{c}^{\circ} g_{+} \cong \mathrm{FL} h_{+} \pi_{T}^{+} \text {and } \mathcal{R}^{\circ} g_{\dagger} \cong \mathrm{FL} h_{\dagger} \pi_{T}^{+},
$$

of functors from $\operatorname{Mod}_{\mathrm{h}}\left(\mathcal{D}_{S_{1}}\right)$ to $\operatorname{Mod}_{\mathrm{h}}\left(\mathcal{D}_{V}\right)$. Notice that although in general $\mathcal{R}_{c}^{\circ}$ and $\mathcal{R}^{\circ}$ are not exact, so the compositions $\mathcal{R}_{c}^{\circ} g_{+}$and $\mathcal{R}^{\circ} g_{\dagger}$ are precisely due to the above isomorphisms.

Applying $\mathrm{FL}_{S_{2}}^{\text {loc }} j_{V^{*}}^{+}$to the natural transformations in 14 we obtain the following ones of functors from $\operatorname{Mod}_{\mathrm{h}}\left(\mathcal{D}_{S_{1}}\right)$ to $\operatorname{Mod}_{\mathrm{h}}\left(\mathcal{D}_{\mathrm{A}_{z}^{1} \times S_{2}}\right)$ :

$$
\mathrm{FL}_{S_{2}}^{\mathrm{loc}} j_{V^{*}}^{+} \mathcal{R}_{c}^{\circ} g_{+} \cong \mathrm{FL}_{S_{2}}^{\mathrm{loc}} j_{V^{*}}^{+} \mathrm{FL} h_{+} \pi^{+} \text {and } \mathrm{FL}_{S_{2}}^{\text {loc }} j_{V^{*}}^{+} \mathcal{R}^{\circ} g_{\dagger} \cong \mathrm{FL}_{S_{2}}^{\text {loc }} j_{V^{*}}^{+} \mathrm{FL} h_{\dagger} \pi^{+}
$$

We are now closer to the isomorphism of the statement; let us rewrite in a different way the functors above to obtain it. Namely,

$\mathrm{FL}_{S_{2}}^{\text {loc }} j_{V^{*}}^{+} \mathrm{FL} \cong j_{V^{*}}^{+} \mathrm{FL}_{W}^{\mathrm{loc}} \mathrm{FL} \cong j_{V^{*}}^{+}\left(j_{z} \times \mathrm{id}_{W}\right)_{+}\left(j_{\tau} \times \mathrm{id}_{W}\right)^{+} \mathrm{FL}{ }_{W} \mathrm{FL} \cong j_{V^{*}}^{+}\left(j_{z} \times \mathrm{id}_{W}\right)_{+}\left(j_{\tau} \times \mathrm{id}_{W}\right)^{+} \mathrm{FL}_{\mathbb{A}_{w_{0}}^{1}}$.

From the third of the assumptions 3.1 we know that we can decompose the morphism $h$ as

$$
h: T \stackrel{h_{1}}{\longrightarrow} \mathbb{G}_{m, w_{0}} \times W^{\vee} \stackrel{j_{\tau} \times \operatorname{id}_{W} \vee}{\longrightarrow} \mathbb{A}_{w_{0}}^{1} \times W^{\vee}=V^{\vee},
$$

where $h_{1}$ is a closed embedding (cf. [RS15b, Prop. 2.1 (1), proof of Thm. 2.4, p. 213]). (Recall that $w_{0}=-\tau$.) It follows that

$\left(j_{\tau} \times \mathrm{id}_{W}\right)^{+} \mathrm{FL}_{\mathbb{A}_{w_{0}}^{1}} h_{+} \cong \mathrm{FL}_{\mathbb{A}_{w_{0}}^{1}}\left(j_{\tau} \times \operatorname{id}_{W} \vee\right)^{+} h_{+} \cong \mathrm{FL}_{\mathbb{A}_{w_{0}}^{1}}\left(j_{\tau} \times \mathrm{id}_{W^{\vee}}\right)^{+}\left(j_{\tau} \times \mathrm{id}_{W} \vee\right)_{+} h_{1,+} \cong \mathrm{FL}_{\mathbb{A}_{w_{0}}^{1}} h_{1,+} \cdot$

Summarizing, we obtain that

$$
\mathrm{FL}_{S_{2}}^{\mathrm{loc}} j_{V^{*}}^{+} \mathrm{FL} h_{\star} \cong j_{V^{*}}^{+}\left(j_{z} \times \mathrm{id}_{W}\right)_{+} \mathrm{FL}_{\mathbb{A}_{w_{0}}^{1}} h_{1,+},
$$

where $\star \in\{+, \dagger\}$, because $h_{1,+}=h_{1, \dagger}$ for $h_{1}$ is proper. In particular, we have $\mathrm{FL}_{S_{2}}^{\text {loc }} \circ j_{V^{*}}^{+} \circ \mathrm{FL} \circ h_{+} \cong$ $\mathrm{FL}_{S_{2}}^{\text {loc }} \circ j_{V^{*}}^{+} \circ \mathrm{FL} \circ h_{\dagger}$. As a consequence, we can claim using the isomorphisms in 15 that

$$
\mathrm{FL}_{S_{2}}^{\text {loc }} j_{V^{*}}^{+} \mathcal{R}_{c}^{\circ} g_{+} \cong \mathrm{FL}_{S_{2}}^{\text {loc }} j_{V^{*}}^{+} \mathcal{R}^{\circ} g_{\dagger},
$$

and so, from Proposition 3.14 and applying this last natural transformation to $\mathcal{O}_{S_{1}}^{\beta}$,

$$
\mathrm{FL}_{S_{2}}^{\text {loc }} j_{V^{*}}^{+} \mathcal{R} g_{\dagger+} \mathcal{O}_{S_{1}}^{\beta} \cong \mathrm{FL}_{S_{2}}^{\text {loc }} j_{V^{*}}^{+} \mathcal{R} g_{+} \mathcal{O}_{S_{1}}^{\beta}
$$

In conclusion, we finally obtain, by using the isomorphisms from 13 as well as the exactness of the functor $\mathrm{FL}_{S_{2}}^{\text {loc }}$, that

$$
\mathrm{FL}_{S_{2}}^{\text {loc }} \mathcal{H}^{0} p_{2,+} k_{\dagger+} \mathcal{O}_{S_{1} \times S_{2}}^{\beta} \cong \mathrm{FL}_{S_{2}}^{\text {loc }} \mathcal{H}^{0} \varphi_{+} \mathcal{O}_{S_{1} \times S_{2}}^{\beta}
$$

The last statement is an easy consequence of isomorphisms in (12) and (13), noting that

$$
\mathrm{FL}_{S_{2}}^{\text {loc }} \mathcal{H}^{0} p_{2,+} k_{\dagger+} \mathcal{O}_{S_{1} \times S_{2}}^{\beta} \cong \mathrm{FL}_{S_{2}}^{\text {loc }} \mathcal{H}^{0} j_{V^{*}}^{+} \mathcal{R} g_{\dagger+} \mathcal{O}_{S_{1}}^{\beta} \cong \mathrm{FL}_{S_{2}}^{\text {loc }} j_{V^{*}}^{+} \mathcal{R} g_{+} \mathcal{O}_{S_{1}}^{\beta} \cong \mathrm{FL}_{S_{2}}^{\text {loc }} \mathcal{H}^{0} \varphi_{+} \mathcal{O}_{S_{1} \times S_{2}}^{\beta}
$$

is induced from the canonical morphism $g_{\uparrow+} \mathcal{O}_{S_{1}}^{\beta} \hookrightarrow g_{+} \mathcal{O}_{S_{1}}^{\beta}$ via $\mathrm{FL}_{S_{2}}^{\text {loc }} j_{V^{*}}^{+} \mathcal{R}$. 
Consider again the space $P=\mathbb{P}^{n} \times\left(\mathbb{A}_{\lambda_{0}}^{1} \times S_{2}\right)$, together with the canonical closed embedding $i_{P}: Z^{*} \hookrightarrow P$. We have the diagram

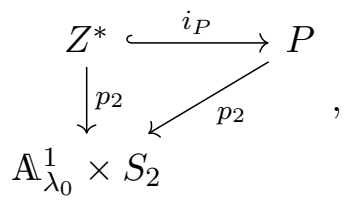

where we denote the restriction of the projection $p_{2}: P \rightarrow \mathbb{A}_{\lambda_{0}}^{1} \times S_{2}$ to $Z^{*}$ by the same letter (as was done before). Since $i_{P}$ is proper, we have

$$
\begin{gathered}
\mathcal{H}^{0} p_{2,+} k_{\dagger+} \mathcal{O}_{S_{1} \times S_{2}}^{\beta} \cong \mathcal{H}^{0} p_{2,+}\left(i_{P} \circ k\right)_{\dagger+} \mathcal{O}_{S_{1} \times S_{2}}^{\beta} \quad \text { and } \\
\mathcal{H}^{0} \varphi_{+} \mathcal{O}_{S_{1} \times S_{2}}^{\beta} \cong \mathcal{H}^{0} p_{2,+} k_{+} \mathcal{O}_{S_{1} \times S_{2}}^{\beta} \cong \mathcal{H}^{0} p_{2,+}\left(i_{P} \circ k\right)_{+} \mathcal{O}_{S_{1} \times S_{2}}^{\beta} .
\end{gathered}
$$

so that we can also write the morphism $\mathcal{H}^{0} p_{2,+} k_{\dagger+} \mathcal{O}_{S_{1} \times S_{2}}^{\beta} \longrightarrow \mathcal{H}^{0} \varphi_{+} \mathcal{O}_{S_{1} \times S_{2}}^{\beta}$ from the proof of Proposition 3.12 as

$$
\mathcal{H}^{0} p_{2,+}\left(i_{P} \circ k\right)_{\dagger+} \mathcal{O}_{S_{1} \times S_{2}}^{\beta} \longrightarrow \mathcal{H}^{0} p_{2,+}\left(i_{P} \circ k\right)_{+} \mathcal{O}_{S_{1} \times S_{2}}^{\beta} .
$$

In order to proceed, we will have to take into account certain group actions on the spaces $S_{1} \times S_{2}$, $P$ and $\mathbb{A}_{\lambda_{0}}^{1} \times S_{2}$ as well as some equivariance properties of the various sheaves of modules on these spaces. For the reader's convenience, we recall some facts from [RS17, § 2.4].

We consider the action of $S_{1}$ on $S_{2}$ given by

$$
\begin{aligned}
\mu: S_{1} \times S_{2} & \longrightarrow S_{2} \\
\left(y_{1}, \ldots, y_{d}, \lambda_{1}, \ldots, \lambda_{n}\right) & \longmapsto\left(\underline{t}^{-\underline{a}_{1}} \lambda_{1}, \ldots, \underline{t}^{-\underline{a}_{n}} \lambda_{n}\right),
\end{aligned}
$$

inducing the following three:

$$
\begin{aligned}
S_{1} \times\left(S_{1} \times S_{2}\right) & \longrightarrow S_{1} \times S_{2} \\
\left(\underline{t},\left(y_{1}, \ldots, y_{d}, \lambda_{1}, \ldots, \lambda_{n}\right)\right) & \longmapsto\left(t_{1} y_{1}, \ldots, t_{d} y_{d}, \underline{t}^{-\underline{a}_{1}} \lambda_{1}, \ldots, \underline{t}^{-\underline{a}_{n}} \lambda_{n}\right), \\
S_{1} \times P & \longrightarrow P=\mathbb{P}^{n} \times\left(\mathbb{A}_{\lambda_{0}}^{1} \times S_{2}\right) \\
\left(\underline{t},\left(\left(w_{0}: \ldots: w_{n}\right), \lambda_{0}, \lambda_{1}, \ldots, \lambda_{n}\right)\right) & \longmapsto\left(\left(w_{0}: \underline{t}^{\underline{a}} w_{1}: \ldots: \underline{t}^{\underline{a}} w_{n}\right), \lambda_{0}, \underline{t}^{-\underline{a}_{1}} \lambda_{1}, \ldots, \underline{t}^{-\underline{a}_{n}} \lambda_{n}\right), \\
S_{1} \times\left(\mathbb{A}_{\lambda_{0}}^{1} \times S_{2}\right) & \longrightarrow \mathbb{A}_{\lambda_{0}}^{1} \times S_{2} \\
\left(\underline{t},\left(\lambda_{0}, \lambda_{1}, \ldots, \lambda_{n}\right)\right) & \longmapsto\left(\lambda_{0}, \underline{t}^{-\underline{a}_{1}} \lambda_{1}, \ldots, \underline{t}^{-\underline{a}_{n}} \lambda_{n}\right) .
\end{aligned}
$$

It is easy to see that all these four actions are free, basically because $\mu$ is free and that they all have smooth geometric quotients. These are described by the following result, which we cite from [RS17, §2.4]. For a free action of an algebraic group $G$ on a smooth variety $X$ admitting a geometric quotient, we write $X / G$ for such quotient.

Proposition 3.15. In the above situation, put'S $:=\left(\mathbb{G}_{m, t}\right)^{n-d}$. Then the geometric quotients $\left(S_{1} \times\right.$ $\left.S_{2}\right) / S_{1}, P / S_{1}$ and $\left(\mathbb{A}_{\lambda_{0}}^{1} \times S_{2}\right) / S_{1}$ are given, respectively, by the spaces

$$
S_{1} \times{ }^{\prime} S,{ }^{\prime} P:=\mathbb{P}^{n} \times \mathbb{A}_{\lambda_{0}}^{1} \times{ }^{\prime} S \text {, and } \mathbb{A}_{\lambda_{0}}^{1} \times{ }^{\prime} S .
$$

There is a canonical embedding' $S \hookrightarrow S_{2}$ inducing embeddings (all denoted by $\iota$ )

$$
\begin{aligned}
S_{1} \times ' S & \hookrightarrow S_{1} \times S_{2}, \\
' P & \hookrightarrow P=\mathbb{P}^{n} \times \mathbb{A}_{\lambda_{0}}^{1} \times S_{2}, \\
S_{1} \times \mathbb{A}_{\lambda_{0}}^{1} \times ' S & \hookrightarrow S_{1} \times \mathbb{A}_{\lambda_{0}}^{1} \times S_{2} .
\end{aligned}
$$

In the sequel, we will always consider ' $S$ as a subvariety of $S_{1}$ (as well as 'P as a subvariety of $P$ etc.).

There is a more direct description of the construction of 'S resp. of the embedding $\iota$ which we recall for the reader's convenience. Namely, let $B$ a Gale dual of $A$ (that is, an integer matrix 
$B \in \mathrm{M}(n \times(n-d), \mathbb{Z})$ whose columns generate $\operatorname{ker}_{\mathbb{Q}}(A)$; see Proposition 2.8). Then we have the split exact sequence of abelian groups

$$
0 \longrightarrow \mathbb{Z}^{n-d} \stackrel{B \cdot}{\longrightarrow} \mathbb{Z}^{n} \stackrel{-A}{\longrightarrow} \mathbb{Z}^{d} \longrightarrow 0 .
$$

By applying the functor $\operatorname{Hom}_{\mathbb{Z}}\left(\bullet, \mathbb{G}_{m}\right)$ to that sequence, we get the exact sequence of algebraic groups

$$
(1) \longrightarrow S_{1}=\mathbb{G}_{m}^{d} \longrightarrow S_{2}=\mathbb{G}_{m}^{n} \longrightarrow \mathbb{G}_{m}^{n-d} \longrightarrow(1),
$$

where the first nontrivial morphism is just $\mu(\bullet, \underline{1})$. From this it follows that the torus $\mathbb{G}_{m}^{n-d}$ can be canonically identified with the geometric quotient $S_{2} / S_{1}$. Since this exact sequence of algebraic tori also splits, we can chose a section $\iota:{ }^{\prime} S=S_{2} / S_{1} \hookrightarrow S_{2}$, and this choice corresponds exactly to the one in RS17, §2.4].

We define reduced versions of the maps $\varphi$ and $i_{P} \circ k$ by the cartesian diagrams

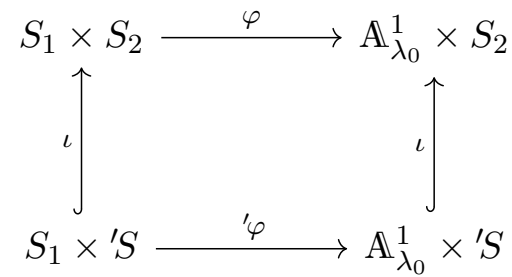

and

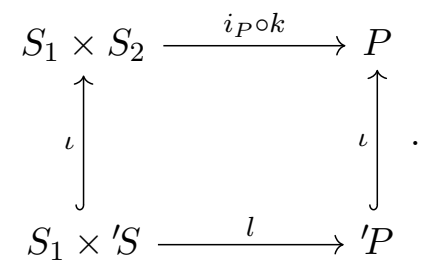

The mapping ' $\varphi: S_{1} \times{ }^{\prime} S \rightarrow \mathbb{A}_{\lambda_{0}}^{1} \times{ }^{\prime} S$ is a family of Laurent polynomials parametrized by 'S, and we denote by 'f $: S_{1} \times{ }^{\prime} S \rightarrow \mathbb{A}_{\lambda_{0}}^{1}$ its first component. In order to illustrate these statements, consider the main case of interest, where we have

$$
A=\left(\begin{array}{cccccc}
1 & -1 & 0 & 0 & \ldots & 0 \\
1 & 0 & -1 & 0 & \ldots & 0 \\
\vdots & \ldots & & & & \\
1 & 0 & 0 & 0 & \ldots & -1
\end{array}\right) \in \mathrm{M}((n-1) \times n, \mathbb{Z}) .
$$

Then $d=n-1,{ }^{\prime} S=\mathbb{G}_{m, t}$ and we can choose

$$
\begin{aligned}
\iota:{ }^{\prime} S=\mathbb{G}_{m, t} & \longrightarrow S_{2}=\mathbb{G}_{m}^{n} \\
t & \longmapsto(t, 1, \ldots, 1) .
\end{aligned}
$$

Then we have (see also the explanation on page 7)

$$
\begin{aligned}
& { }^{\prime} \varphi: S_{1} \times \mathbb{G}_{m, t} \longrightarrow \mathbb{A}_{\lambda_{0}}^{1} \times \mathbb{G}_{m, t} \\
& \left(y_{1}, \ldots, y_{n-1}, t\right) \longmapsto\left(-\frac{1}{y_{1}}-\ldots-\frac{1}{y_{n-1}}-t \cdot y_{1} \cdot \ldots \cdot y_{n-1}, t\right)
\end{aligned}
$$

Going back to the situation of a general matrix $A \in \mathrm{M}(d \times n, \mathbb{Z})$ satisfying the assumptions 3.1. we state the following result, inspired from RS17, showing that $\iota$ behaves well with respect to all modules in question. To simplify our notation we will write

$$
M_{\dagger+, S_{2}}:=\mathcal{H}^{0} p_{2,+}\left(i_{P} \circ k\right)_{\dagger+} \mathcal{O}_{S_{1} \times S_{2}}^{\beta} \quad \text { and } \quad M_{S_{2}}:=\mathcal{H}^{0} \varphi_{+} \mathcal{O}_{S_{1} \times S_{2}}^{\beta}=\mathcal{H}^{0} p_{2,+}\left(i_{P} \circ k\right)_{+} \mathcal{O}_{S_{1} \times S_{2}}^{\beta},
$$

and analogously,

$$
M_{\dagger+}:=\mathcal{H}^{0 \prime} p_{2,+} l_{\dagger+} \mathcal{O}_{S_{1} \times ' S}^{\beta} \quad \text { and } \quad M:=\mathcal{H}^{0 \prime} \varphi_{+} \mathcal{O}_{S_{1} \times ' S}^{\beta}=\mathcal{H}^{0} p_{2,+} l_{+} \mathcal{O}_{S_{1} \times ' S}^{\beta},
$$

where we write $p_{2}:{ }^{\prime} P=\mathbb{P}^{n} \times \mathbb{A}_{\lambda_{0}}^{1} \times{ }^{\prime} S \rightarrow \mathbb{A}_{\lambda_{0}}^{1} \times{ }^{\prime} S$ (to distinguish this projection from the one to $\mathbb{A}_{\lambda_{0}}^{1} \times S_{2}$ considered above).

Proposition 3.16. We have a morphism of $\mathcal{D}_{\mathbb{A}_{\lambda_{0}}^{1} \times{ }^{\prime} S}$-modules

$$
\phi: M_{\dagger+} \longrightarrow M
$$

inducing an isomorphism of $\mathcal{D}_{\mathbb{A}_{z}^{1} \times^{\prime} S}$-modules

$$
\mathrm{FL}_{S_{S}}^{\mathrm{loc}} M_{\dagger+} \cong \mathrm{FL}_{S_{S}}^{\mathrm{loc}} M .
$$


Moreover, both modules $M$ and $M_{\dagger+}$ are non-characteristic with respect to the projection ${ }^{\prime} p:{ }^{\prime} P \rightarrow$ $\mathbb{A}_{\lambda_{0}}^{1} \times{ }^{\prime} S$ along the subspace $P \backslash{ }^{\prime} P^{*}$, where $P^{*}=\mathbb{A}^{n} \times\left(\mathbb{A}_{\lambda_{0}}^{1} \times ' S\right)$.

Proof. It has been shown in RS17, Prop. 2.22., Lem. 6.4.] that the morphism $\iota: \mathbb{A}_{\lambda_{0}}^{1} \times{ }^{\prime} S \hookrightarrow \mathbb{A}_{\lambda_{0}}^{1} \times S_{2}$ is non-characteristic for the modules $M_{\dagger+, S_{2}}$ and $M_{S_{2}}$. Consequently, we obtain the morphism of $\mathcal{D}_{\mathbb{A}_{\lambda_{0}}^{1} \times S^{\prime}}$-modules

$$
\phi: M_{\dagger+} \longrightarrow M
$$

by applying $\iota^{+}$to the morphism

$$
M_{\dagger+, S_{2}}=\mathcal{H}^{0} p_{2,+}\left(i_{P} \circ k\right)_{\dagger+} \mathcal{O}_{S_{1} \times S_{2}}^{\beta} \longrightarrow \mathcal{H}^{0} \varphi_{+} \mathcal{O}_{S_{1} \times S_{2}}^{\beta}=\mathcal{H}^{0} p_{2,+}\left(i_{P} \circ k\right)_{+} \mathcal{O}_{S_{1} \times S_{2}}^{\beta}=M_{S_{2}}
$$

(see (16)). Since both the functor $\mathrm{FL}_{S_{2}}^{\text {loc }}$ and the functor $\mathrm{FL}_{S_{S}}^{\text {loc }}$ commute with $\iota$, we obtain the isomorphism $\mathrm{FL}_{S_{S}}^{\text {loc }} M_{\dagger+} \cong \mathrm{FL}_{S_{S}}^{\text {loc }} M$.

Finally, the fact that $\iota$ is non-characteristic for $M_{\dagger+, S_{2}}$ and $M_{S_{2}}$ yields the last statement using Lemma 3.9 .

Remark 3.17. From now on we will assume that the $\beta_{i}$ are real numbers, since we will use some Hodge theoretic constructions, which are not valid for arbitrary complex $\beta_{i}$, as commented in Remark 3.6.

We start by introducing certain auxiliary filtrations on the $\mathcal{D}$-modules considered above which are not a priori Hodge filtrations but have an explicit description. We will see later that it is sufficient to consider these filtrations to extract the Hodge theoretic information we need.

Recall that an easy calculation decomposing ' $\varphi$ as a graph embedding followed by a projection shows that the direct image complex ${ }^{\prime} \varphi_{+} \mathcal{O}_{S_{1} \times{ }^{\prime} S}^{\beta}$ can be represented by

$$
\left({ }^{\prime} \varphi_{*} \Omega_{S_{1} \times ' S / S}^{\bullet+d}\left[\partial_{\lambda_{0}}\right], d-\kappa(\beta) \wedge-\left(d^{\prime} f \wedge\right) \otimes \partial_{\lambda_{0}}\right),
$$

where ' $f$ is still the first component of ' $\varphi$.

We consider the filtration on each ' $\varphi_{*} \Omega_{S_{1} \times{ }^{\prime} S / S}^{l+d}\left[\partial_{\lambda_{0}}\right]$ given by

$$
F_{k+l}{ }^{\prime} \varphi_{*} \Omega_{S_{1} \times{ }^{\prime} S / S}^{l+d}\left[\partial_{\lambda_{0}}\right]=\sum_{i=0}^{k+l}{ }^{\prime} \varphi_{*} \Omega_{S_{1} \times^{\prime} S /{ }^{\prime} S}^{l+d} \partial_{\lambda_{0}}^{i} .
$$

One easily checks that this filtration is compatible with the differential, so that we obtain the filtered complex

$$
\begin{aligned}
& F_{k}\left({ }^{\prime} \varphi_{*} \Omega_{S_{1} \times{ }^{\prime} S /{ }^{\prime} S}^{\bullet+d}\left[\partial_{\lambda_{0}}\right], d-(\kappa(\beta) \wedge)-\left(d^{\prime} f \wedge\right) \otimes \partial_{\lambda_{0}}\right):= \\
& \left(F_{k+\bullet} \varphi^{\prime} \varphi_{*} \Omega_{S_{1} \times^{\prime} S /{ }^{\prime} S}\left[\partial_{\lambda_{0}}\right], d-(\kappa(\beta) \wedge)-\left(d^{\prime} f \wedge\right) \otimes \partial_{\lambda_{0}}\right) \text {. }
\end{aligned}
$$

Definition 3.18. We call the induced filtration $F_{\bullet} \mathcal{H}^{i \prime} \varphi_{+} \mathcal{O}_{S_{1} \times ' S}^{\beta}$ on the cohomology sheaves $\mathcal{H}^{i \prime} \varphi_{+} \mathcal{O}_{S_{1} \times ' S}^{\beta}$ the Brieskorn filtration.

Notice that for $i=0, k=0$ and $\beta=0$ the filtration step $F_{k} \mathcal{H}^{0} \varphi_{+} \mathcal{O}_{S_{1} \times{ }^{\prime} S}^{\beta}=F_{0} M$ is exactly the module $M_{0}$ considered in [Sab06] and was called Brieskorn lattice there because it was defined similarly to the case of isolated hypersurface singularities (see [Bri70]).

On the other hand, it is well-known that $l_{\dagger+} \mathcal{O}_{S_{1} \times^{\prime} S}^{\beta}$ carries a filtration $F^{H} l_{\dagger+} \mathcal{O}_{S_{1} \times^{\prime} S}^{\beta}$ such that $\left(l_{\dagger+} \mathcal{O}_{S_{1} \times^{\prime} S}^{\beta}, F^{H}\right)$ underlies a pure polarizable complex Hodge module of weight $n=\operatorname{dim}\left(S_{1}\right)+$ $\operatorname{dim}\left({ }^{\prime} S\right)$, in particular, an element of $\operatorname{MHM}\left({ }^{\prime} P, \mathbb{C}\right)$. Since $p_{2}$ is projective, the direct image $M_{\dagger+}=$ $\mathcal{H}^{0 \prime} p_{2,+} l_{\dagger+} \mathcal{O}_{S_{1} \times^{\prime} S}^{\beta}$ also carries a Hodge filtration $F^{H} \mathcal{H}^{0 \prime} p_{2,+} l_{\dagger+} \mathcal{O}_{S_{1} \times^{\prime} S}^{\beta}$ such that $\left(M_{\dagger+}, F^{H}\right)$ underlies a pure polarisable complex Hodge module (see the first point of [Sai88, Thm. 1]). We consider the shifted filtration $F_{\bullet}^{H_{s h}} M_{\dagger+}:=F_{\bullet-d}^{H} M_{\dagger+}$. The next step is to compare this filtration to the Brieskorn filtration on $M$ via the morphism $\phi$. We will show in the next lemma that $\phi$ is filtered. Notice that since the Brieskorn filtration has a priori no Hodge properties, we cannot simply deduce this result from the functorial properties of mixed Hodge modules. 
Lemma 3.19. Consider again the morphism $\phi: M_{\dagger+} \longrightarrow M$ from Proposition 3.16 which yields an isomorphism of $\mathcal{D}_{\mathbb{A}_{z}^{1} \times ' S}$-modules after applying the functor $\mathrm{FL}_{S}^{\text {loc }}$. Then $\phi$ is filtered with respect to the shifted Hodge filtration on $M_{\dagger+}$ and with respect to the Brieskorn filtration on $M$, i.e., for every $k \in \mathbb{Z}$, we have the inclusion

$$
\phi\left(F_{k}^{H_{s h}} M_{\dagger+}\right) \subset F_{k} M
$$

Proof. Recall that ' $P^{*}=\mathbb{A}^{n} \times\left(\mathbb{A}_{\lambda_{0}}^{1} \times{ }^{\prime} S\right)$ is the complement in 'P of the divisor $w_{0}=0$. Then the map $l$ can be decomposed into a closed embedding $l_{1}: S_{1} \times{ }^{\prime} S \hookrightarrow{ }^{\prime} P^{*}$ and an open embedding $l_{2}:{ }^{\prime} P^{*} \hookrightarrow^{\prime} P$. Here again the fact that $l_{1}$ is closed follows from the third assumption in 3.1. We can further decompose $l_{1}$ as $l_{1}=l_{0} \circ i_{\varphi}$, , where $i_{\varphi}{ }_{\varphi}$ is the graph embedding of ' $\varphi$. More precisely, we have the following diagram:

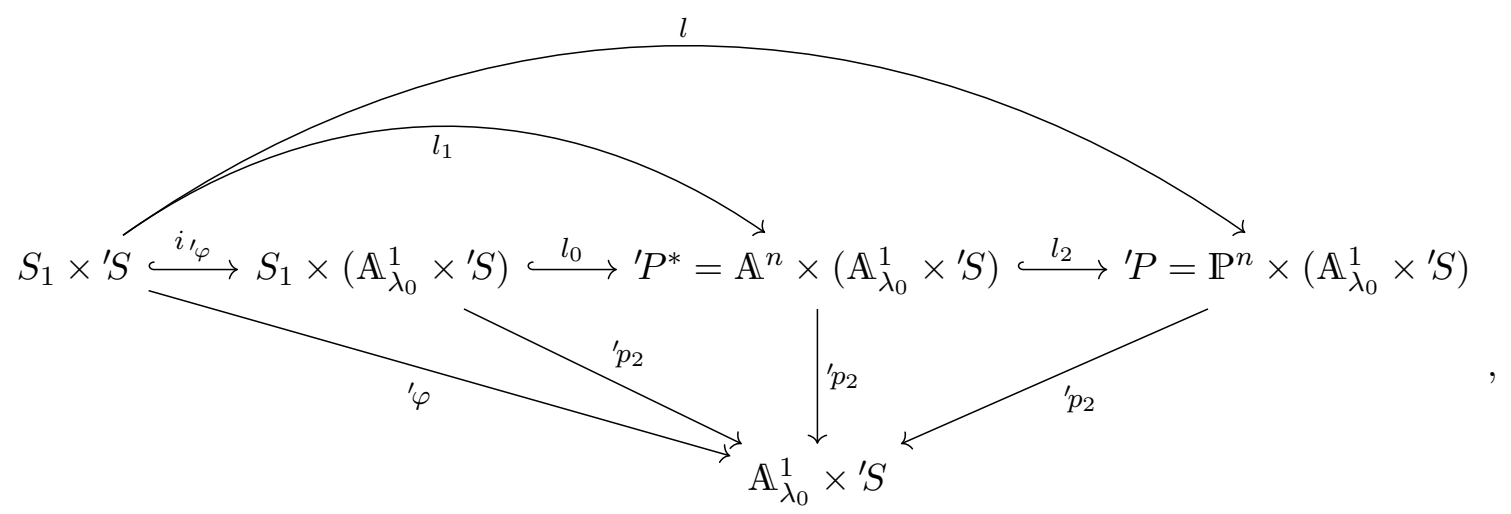

where we denote by slight abuse of notation all projections to the last coordinates by ${ }^{\prime} p_{2}$. Recall that $M_{\dagger+}=\mathcal{H}^{0 \prime} p_{2,+} l_{\dagger+} \mathcal{O}_{S_{1} \times^{\prime} S}^{\beta}$ and $M=\mathcal{H}^{0} \varphi_{+} \mathcal{O}_{S_{1} \times^{\prime} S}^{\beta}=\mathcal{H}^{0 \prime} p_{2,+} l_{1,+} \mathcal{O}_{S_{1} \times^{\prime} S}^{\beta}$.

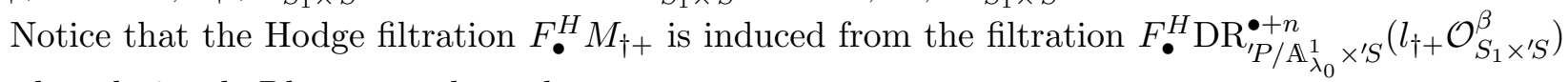
on the relative de Rham complex, where

$$
F_{k}^{H} \mathrm{DR}_{I P / \mathbb{A}_{\lambda_{0}}^{1} \times^{\prime} S}^{\bullet+n}\left(l_{\dagger+} \mathcal{O}_{S_{1} \times^{\prime} S}^{\beta}\right):=\left(\ldots \longrightarrow \Omega_{I^{\prime} / \mathbb{A}_{\lambda_{0}}^{1} \times^{\prime} S}^{n+l} \otimes F_{k+n+l}^{H} l_{\dagger+} \mathcal{O}_{S_{1} \times^{\prime} S}^{\beta} \longrightarrow \ldots\right)
$$

by the first point of [Sai88, Thm. 1].

On the other hand, we have the isomorphism of $\mathcal{D}_{\mathbb{A}_{\lambda_{0}}^{1} \times{ }^{\prime}}$-modules

$$
\mathcal{H}^{0 \prime} p_{2, *} \mathrm{DR}_{P^{*} / \mathbb{A}_{\lambda_{0}}^{1} \times^{\prime} S}^{n+\cdot}\left(l_{1,+} \mathcal{O}_{S_{1} \times^{\prime} S}^{\beta}\right) \cong M
$$

as the above diagram shows, just by the definition of $\mathcal{H}^{0 \prime} p_{2,+}$. The Hodge filtration $F_{\bullet}^{H}$ on the module $l_{1,+} \mathcal{O}_{S_{1} \times^{\prime} S}^{\beta}$ can be explicitly written down, due to the fact that $l_{1}$ is a closed embedding (in particular, the filtered module $\left(l_{1,+} \mathcal{O}_{S_{1} \times^{\prime} S}^{\beta}, F_{\bullet}^{H}\right)$ underlies a pure Hodge module), and then one checks that the shifted Brieskorn filtration $F_{\bullet}[-d]:=F_{\bullet+d}$ on the module $M$ (see formula (18) for the definition of the Brieskorn filtration) is induced from the filtration

$$
F_{k}^{H} \mathrm{DR}_{P^{*} / \mathbb{A}_{\lambda_{0}}^{1} \times{ }^{\prime} S}^{\bullet+n}\left(l_{1,+} \mathcal{O}_{S_{1} \times^{\prime} S}^{\beta}\right):=\left(\ldots \longrightarrow \Omega_{P^{*} / \mathbb{A}_{\lambda_{0}}^{1} \times^{\prime} S}^{n+l} \otimes F_{k+n+l}^{H} l_{1,+} \mathcal{O}_{S_{1} \times^{\prime} S}^{\beta} \longrightarrow \ldots\right)
$$

Notice once again that we cannot deduce from this description that the Brieskorn filtration $F_{\bullet} M$ (or its shifted version $\left.F_{\bullet}[-d] M\right)$ has any Hodge properties since the map ${ }^{\prime} p_{2}:{ }^{\prime} P^{*} \rightarrow \mathbb{A}_{\lambda_{0}}^{1} \times{ }^{\prime} S$ is not projective (and in particular the filtration from formula 18 on the direct image complex ${ }^{\prime} \varphi_{+} \mathcal{O}_{S_{1} \times{ }^{\prime} S}^{\beta}$ is not necessarily strict).

We will now show that the induced morphism on global sections

$$
\phi: \Gamma\left(\mathbb{A}_{z}^{1} \times{ }^{\prime} S, M_{\dagger+}\right) \longrightarrow \Gamma\left(\mathbb{A}_{z}^{1} \times{ }^{\prime} S, M\right)
$$

is filtered, i.e. sends the $\Gamma\left(\mathbb{A}_{z}^{1} \times{ }^{\prime} S, \mathcal{O}_{\mathbb{A}_{z}^{1} \times ' S}\right)$-submodule $\Gamma\left(\mathbb{A}_{z}^{1} \times{ }^{\prime} S, F_{k}^{H_{s h}} M_{\dagger+}\right)$ to $\Gamma\left(\mathbb{A}_{z}^{1} \times{ }^{\prime} S, F_{k} M\right)$. This is obviously equivalent to the statement of the proposition since $\mathbb{A}_{z}^{1} \times{ }^{\prime} S$ is affine. 
We have (denoting $a: \mathbb{A}_{z}^{1} \times{ }^{\prime} S \rightarrow\{p t\}$ )

$$
\begin{aligned}
& \Gamma\left(\mathbb{A}_{z}^{1} \times{ }^{\prime} S, M_{\dagger+}\right)=a_{*} M_{\dagger+}=a_{*} \mathcal{H}^{0} \mathbf{R}\left({ }^{\prime} p_{2}\right)_{*} \mathrm{DR}_{{ }^{\prime} P / \mathbb{A}_{\lambda_{0}}^{1} \times{ }^{\prime} S}^{n+}\left(l_{\dagger+} \mathcal{O}_{S_{1} \times^{\prime} S}^{\beta}\right) \\
& =H^{0} \mathbf{R}\left(a \circ^{\prime} p_{2}\right)_{*} \mathrm{DR}_{I P / \mathbb{A}_{\lambda_{0}}^{1} \times{ }^{\prime} S}^{n+\bullet}\left(l_{\dagger+} \mathcal{O}_{S_{1} \times^{\prime} S}^{\beta}\right)=H^{0} \mathbf{R} \Gamma\left({ }^{\prime} P, \mathrm{DR}_{I P / \mathbb{A}_{\lambda_{0}}^{1} \times^{\prime} S}^{n+\bullet}\left(l_{\dagger+} \mathcal{O}_{S_{1} \times{ }^{\prime} S}^{\beta}\right)\right)
\end{aligned}
$$

and similarly

$$
\begin{aligned}
& \Gamma\left(\mathbb{A}_{z}^{1} \times{ }^{\prime} S, M_{+}\right)=a_{*} M_{+}=a_{*} \mathcal{H}^{0} \mathbf{R}\left({ }^{\prime} p_{2}\right)_{*} \mathrm{DR}_{P^{*} / \mathbb{A}_{\lambda_{0}}^{1} \times^{\prime} S}^{n+}\left(l_{1,+} \mathcal{O}_{S_{1} \times^{\prime} S}^{\beta}\right) \\
& =H^{0} \mathbf{R}\left(a \circ^{\prime} p_{2}\right)_{*} \mathrm{DR}_{\prime P^{*} / \mathbb{A}_{\lambda_{0}}^{1} \times^{\prime} S}^{n+\bullet}\left(l_{1,+} \mathcal{O}_{S_{1} \times^{\prime} S}^{\beta}\right)=H^{0} \mathbf{R} \Gamma\left({ }^{\prime} P^{*}, \mathrm{DR}_{\prime P^{*} / \mathbb{A}_{\lambda_{0}}^{1} \times^{\prime} S}^{n+\bullet}\left(l_{1,+} \mathcal{O}_{S_{1} \times^{\prime} S}^{\beta}\right)\right) \\
& =H^{0} \Gamma\left({ }^{*} P^{*}, \mathrm{DR}_{\prime}^{\prime P^{*} / \mathrm{A}_{\lambda_{0}}^{1} \times{ }^{\prime} S}\left(l_{1,+} \mathcal{O}_{S_{1} \times{ }^{\prime} S}^{\beta}\right)\right)
\end{aligned}
$$

(for the last equality, we use that ' $P^{*}$ is affine, in contrast to ${ }^{\prime} P$ ). Now notice that the morphism $\phi: \Gamma\left(\mathbb{A}_{z}^{1} \times{ }^{\prime} S, M_{\dagger+}\right) \longrightarrow \Gamma\left(\mathbb{A}_{z}^{1} \times{ }^{\prime} S, M\right)$ is induced from

$$
\begin{aligned}
& \mathbf{R} \Gamma\left({ }^{\prime} P, \mathrm{DR}_{{ }^{\prime} P / \mathbb{A}_{\lambda_{0}}^{1} \times{ }^{\prime} S}^{n+\bullet}\left(l_{\dagger+} \mathcal{O}_{S_{1} \times^{\prime} S}^{\beta}\right)\right) \rightarrow \mathbf{R} \Gamma\left({ }^{\prime} P^{*}, \mathrm{DR}_{{ }^{\prime} P / \mathbb{A}_{\lambda_{0}}^{1} \times{ }^{\prime} S}^{n+\bullet}\left(l_{\dagger+} \mathcal{O}_{S_{1} \times^{\prime} S}^{\beta}\right)\right)
\end{aligned}
$$

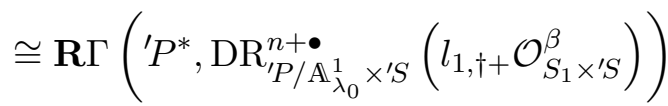

$$
\begin{aligned}
& \cong \mathbf{R} \Gamma\left({ }^{\prime} P^{*}, \mathrm{DR}_{\prime P^{*} / \mathbb{A}_{\lambda_{0}}^{1} \times{ }^{\prime} S}^{n+\bullet}\left(l_{1,+} \mathcal{O}_{S_{1} \times{ }^{\prime} S}^{\beta}\right)\right) \\
& \cong \Gamma\left({ }^{\prime} P^{*}, \mathrm{DR}_{P^{*} / \mathbb{A}_{\lambda_{0}}^{1} \times^{\prime} S}^{n+\bullet}\left(l_{1,+} \mathcal{O}_{S_{1} \times^{\prime} S}^{\beta}\right)\right) \text {, }
\end{aligned}
$$

where the first morphism is simply given by restricting sections of $l_{\dagger+} \mathcal{O}_{S_{1} \times{ }^{\prime} S}^{\beta}$ from 'P to 'P* and the isomorphisms are due to the fact that $l$ restricted to ${ }^{\prime} P^{*}$ is $l_{1}$, which is proper and affine.

Obviously, the restriction of the Hodge filtration on the module $l_{++} \mathcal{O}_{S_{1} \times^{\prime} S}^{\beta}$ is the Hodge filtration on the module $l_{1, \uparrow+} \mathcal{O}_{S_{1} \times{ }^{\prime} S}^{\beta}=l_{1,+} \mathcal{O}_{S_{1} \times^{\prime} S}^{\beta}$. Since, as has been discussed before, the filtration $F_{k}^{H} \mathrm{DR}_{P / \mathbb{A}_{\lambda_{0}}^{1} \times^{\prime} S}^{\bullet+n}\left(l_{\dagger+} \mathcal{O}_{S_{1} \times^{\prime} S}^{\beta}\right)$ induces $F_{\bullet}^{H} M_{\dagger+}$ and the filtration $F_{k}^{H} \mathrm{DR}_{P^{*} / \mathbb{A}_{\lambda_{0}}^{1} \times^{\prime} S}^{\bullet+n}\left(l_{1,+} \mathcal{O}_{S_{1} \times^{\prime} S}^{\beta}\right)$ induces $F_{\bullet}[-d] M$, we conclude that $\phi: \Gamma\left(\mathbb{A}_{z}^{1} \times{ }^{\prime} S, M_{\dagger+}\right) \rightarrow \Gamma\left(\mathbb{A}_{z}^{1} \times{ }^{\prime} S, M\right)$ sends $\Gamma\left(\mathbb{A}_{z}^{1} \times{ }^{\prime} S, F_{k}^{H} M_{\dagger+}\right)$ to $\Gamma\left(\mathbb{A}_{z}^{1} \times{ }^{\prime} S, F_{k}[-d] M\right)$, or, equivalently, $\Gamma\left(\mathbb{A}_{z}^{1} \times{ }^{\prime} S, F_{k}^{H_{s h}} M_{\dagger+}\right)$ to $\Gamma\left(\mathbb{A}_{z}^{1} \times{ }^{\prime} S, F_{k} M\right)$, as required.

In general, the filtrations on $M_{\dagger+}$ and $M$ are not equal, simply because the underlying $\mathcal{D}_{\mathbb{A}_{\lambda_{0}}^{1} \times{ }^{\prime} S^{-}}$ modules are not equal. They become equal after localized partial Fourier transformation as we have shown in Proposition 3.12. First we will explain that these transformations can be performed at the filtered level and how the last result can be interpreted in this context.

We will use a general procedure which produces from a filtered $\mathcal{D}_{\mathrm{A}_{\lambda_{0}}^{1} \times^{\prime} S^{-}}$module $\left(N, F_{\bullet}\right)$ a lattice

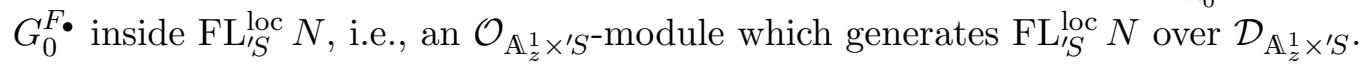

Definition 3.20. (cf. [Sab08, §1.d], [SY15, §A.1]) Let $X$ be a smooth affine variety and let $\left(N, F_{\bullet}\right)$ be a filtered $\mathcal{D}_{\mathrm{A}_{s}^{1} \times X}$-module, which we identify with its module of global sections. Consider the algebraic microlocalization

$$
N\left[\partial_{s}^{-1}\right]:=\mathbb{C}[s]\left\langle\partial_{s}, \partial_{s}^{-1}\right\rangle \otimes_{\mathbb{C}[s]\left\langle\partial_{s}\right\rangle} N .
$$

By letting act $\tau$ as $-\partial_{s}$ and $\partial_{\tau}$ as $s$, we consider $N\left[\partial_{s}^{-1}\right]$ as a $\mathcal{D}_{X}\left[\tau, \tau^{-1}\right]\left\langle\partial_{\tau}\right\rangle$-module (which actually coincides with $\left.\mathrm{FL}_{X}^{\text {loc }} N\right)$. Let now $\widehat{\text { loc }}$ be the natural localization morphism $\widehat{\mathrm{loc}}: N \rightarrow N\left[\partial_{s}^{-1}\right]$. Then we define

$$
G_{0}^{F} \cdot \mathrm{FL}_{X}^{\mathrm{loc}} N:=\sum_{j \geq 0} \partial_{s}^{-j} \widehat{\operatorname{loc}}\left(F_{j} N\right)
$$


notice that then $G_{0}^{F} \cdot \mathrm{FL}_{X}^{\text {loc }} N$ has naturally the structure of a $\mathcal{R}_{\mathbb{A}}^{\mathrm{int} \times X}$-module. We also put for any $k \in \mathbb{Z}$

$$
G_{k}^{F \cdot} \mathrm{FL}_{X}^{\mathrm{loc}} N:=z^{k} \cdot G_{0}^{F \bullet} \mathrm{FL}_{X}^{\operatorname{loc}} N=\sum_{j \geq 0} \partial_{s}^{-(j+k)} \widehat{\operatorname{loc}}\left(F_{j} N\right)=\sum_{j \geq 0} \partial_{s}^{-j} \widehat{\operatorname{loc}}\left(F_{j+k} N\right) .
$$

There is an interpretation of this construction as a Fourier-Laplace transformation for $\mathcal{R}_{\mathbb{A}_{z}^{1} \times \mathbb{A}_{s}^{1} \times X^{-}}^{\text {int }}$ modules as explained in SY15, Rem. A.3]. Using this interpretation, one can show the following fact.

Lemma 3.21. Let $\left(N, F_{\bullet}\right)$ be a filtered $\mathcal{D}_{\mathbb{A}_{s}^{1} \times X}$-module underlying an element in $\operatorname{MHM}\left(\mathbb{A}_{s}^{1} \times X, \mathbb{C}\right)$ (the abelian category of complex mixed Hodge modules). Then the $\mathcal{R}_{\mathbb{A}_{z}^{1} \times X}^{\text {int }}$-module $G_{0}^{F} \cdot \mathrm{FL}_{X}^{\operatorname{loc}}(N)$ underlies an element of $\operatorname{IrrMHM}(X)$.

Proof. We first define the algebraic Fourier-Laplace transformation for integrable $\mathcal{R}$-modules as in CDRS18, Defs. 3.2, 3.7]. Consider the following diagram:

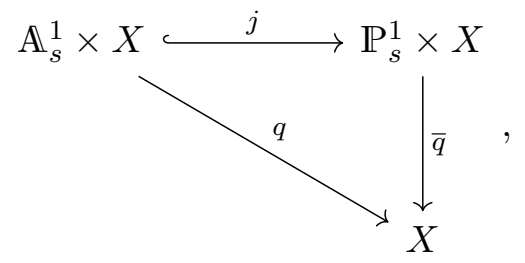

where $j$ is the canonical open embedding and $q$ and $\bar{q}$ the respective second projections. Let $\mathcal{A}_{\text {aff }}^{s / z}$ the $\mathcal{R}_{\mathrm{A}_{z}^{1} \times \mathbb{A}_{s} \times X}$-module $\mathcal{O}_{\mathbb{A}_{z}^{1} \times \mathbb{A}_{s} \times X}$ equipped with the $z$-connection $z d+d s$. Then for an algebraic $\mathcal{R}_{\mathrm{A}_{z}^{1}}^{\text {int }} \times \mathbb{A}_{s}^{1} \times X^{-m o d u l e} \mathcal{N}$, we put

$$
\operatorname{FL}_{X}^{\mathcal{R}}(\mathcal{N}):=\mathcal{H}^{0} q_{+}\left(\mathcal{N} \otimes \mathcal{A}_{\text {aff }}^{s / z}\right) \in \operatorname{Mod}\left(\mathcal{R}_{\mathbb{A}_{z}^{1} \times X}^{\mathrm{int}}\right) .
$$

Let $D$ be the reduced divisor $\left(\mathbb{P}_{s}^{1} \backslash \mathbb{A}_{s}^{1}\right) \times X=\{\infty\} \times X$. Then $\mathcal{A}_{*}^{s / z}:=j_{*} \mathcal{A}_{\text {aff }}^{s / z}$ carries a natural structure of an $\mathcal{R}_{\mathbb{A}_{z}^{1} \times \mathbb{P}_{s}^{1} \times X}(* D)$-module. Let $\mathcal{E}_{*}^{s / z}$ be the analytification of $\mathcal{A}_{*}^{s / z}$.

As in the case of $\mathcal{D}$-modules, there is the notion of strict specializability and $V$-filtration for $\mathcal{R}^{\text {int }}$ modules; check Moc15a, §2.1.2] for more details.

Notice that the meromorphic function $s \in \mathcal{O}_{\mathbb{P}_{s}^{1} \times X}(* D)$ evidently extends to a map $\mathbb{P}_{s}^{1} \times X \rightarrow \mathbb{P}_{s}^{1}$ whose reduced pole divisor is exactly $D \cong X$, in particular, it is smooth. Then it follows from SY15, Lem. 3.1] (using that, according to their notation, $P_{\text {red }}=D$ and $\mathbf{e}=1$ ) that $\mathcal{E}_{*}^{s / z}$ is coherent not only over $\mathcal{R}_{\mathrm{A}_{z}^{1} \times \mathbb{P}_{s}^{1} \times X}$ but over $V_{0} \mathcal{R}_{\mathrm{A}_{z}^{1} \times \mathbb{P}_{s}^{1} \times X}$ too. By $[$ Moc15a, $\S 2.1 .2]$ we know that $\mathcal{E}_{*}^{s / z}$ is automatically specializable along $D$ and its corresponding $V$-filtration is trivial. Therefore, by construction, $\mathcal{E}^{s / z}:=\mathcal{E}_{*}^{s / z}[* D]=\mathcal{E}_{*}^{s / z}$ (cf. [ibid., §3.1.2]). We then know by SY15, Prop. 3.3 that $\mathcal{E}^{s / z}$ underlies an algebraic, integrable pure twistor $\mathcal{D}$-module $\mathcal{T}^{s / z}$ on $\mathbb{A}_{s}^{1} \times X$. (Cf. Sab18a, \$1.6.a] for another discussion on this issue.)

Now suppose that $\mathcal{N}$ underlies an algebraic, integrable mixed twistor $\mathcal{D}$-module $\mathcal{N}=\left(\mathcal{N}^{\prime}, \mathcal{N}, C\right) \in$ $\operatorname{MTM}_{\text {alg }}^{\text {int }}\left(\mathbb{A}_{s}^{1} \times X\right)$, then we can define its relative Fourier-Laplace transform

$$
\operatorname{FL}_{X}^{\mathrm{MTM}}(\mathcal{N}):=\mathcal{H}^{0} q_{*}\left(\mathcal{N} \otimes \mathcal{T}^{s / z}\right) \in \operatorname{MTM}_{\text {alg }}^{\mathrm{int}}(X) .
$$

For notational convenience, for any $\mathcal{R}$-triple $\mathcal{K}=\left(\mathcal{K}^{\prime}, \mathcal{K}, C\right)$ we define the forgetful functor For $(\mathcal{K}):=$ $\mathcal{K}$. Then we have a comparison formula, the proof of which is completely analogous to CDRS18, Prop. $3.5]$ :

Let $\mathcal{N}$ be an element in $\mathrm{MTM}_{\text {alg }}^{\mathrm{int}}\left(\mathbb{A}_{s}^{1} \times X\right)$, then there is an isomorphism of $\mathcal{R}_{\mathrm{A}_{z}^{1} \times X^{-}}$-modules:

$$
\operatorname{For}\left(\operatorname{FL}_{X}^{\mathrm{MTM}}(\mathcal{N})\right) \cong z^{-1} \operatorname{FL}_{X}^{\mathcal{R}}(\operatorname{For}(\mathcal{N})) \text {. }
$$

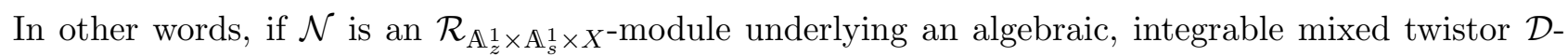
module on $\mathbb{A}_{s}^{1} \times X$, then the (shifted) relative algebraic Fourier-Laplace transform $z^{-1} \mathrm{FL}_{X}^{\mathcal{R}}(\mathcal{N})$ underlies an element of $\operatorname{MTM}_{\text {alg }}^{\text {int }}(X)$. 
Now let us suppose that we are given a filtered $\mathcal{D}_{\mathbb{A}_{s}^{1} \times X^{-}}$-module $\left(N, F_{\bullet}\right)$ underlying a complex mixed Hodge module on $\mathbb{A}_{s}^{1} \times X$. Then its Rees module $\mathcal{R}_{F} \mathcal{N}$ is an algebraic $\mathcal{R}_{\mathbb{A}_{z}^{1} \times \mathbb{A}_{s}^{1} \times X}$-module and underlies an element $\mathcal{N}$ in $\operatorname{MTM}_{\text {alg }}^{\mathrm{int}}\left(\mathbb{A}_{s}^{1} \times X\right)$ as well as in $\operatorname{IrrMHM}\left(\mathbb{A}_{s}^{1} \times X\right)$. Since all the functors entering in the definition of $\mathrm{FL}_{X}^{\mathrm{MTM}}$ preserve the category of irregular Hodge modules by Sab18a, Cor. 0.5.], we know by the above comparison result (i.e. by formula (23)) that $z^{-1} \mathrm{FL}_{X}^{\mathcal{R}}\left(\mathcal{R}^{F} N\right)$ underlies an irregular Hodge module on $X$. Now, by [SY15, Rem. A.3] (noting that the argument there is valid for the partial transformation as well), we can identify $\operatorname{FL}_{X}^{\mathcal{R}}\left(\mathcal{R}^{F} N\right)$ with $z G_{0}^{F \bullet} \operatorname{FL}_{X}^{\text {loc }}(N)$, so that

$$
G_{0}^{F} \cdot \mathrm{FL}_{X}^{\text {loc }}(N) \cong z^{-1} \mathrm{FL}_{X}^{\mathcal{R}}\left(\mathcal{R}^{F} N\right) \text {. }
$$

This shows that $G_{0}^{F} \cdot \mathrm{FL}_{X}^{\text {loc }}(N)$ underlies an irregular Hodge module on $X$, as required.

With these definitions at hand, we have the following consequence of Lemma 3.19 .

Corollary 3.22. In the above situation, we have

$$
G_{0}^{F_{\bullet}^{H_{s h}}} \mathrm{FL}_{{ }_{S}}^{\text {loc }} M_{\dagger+} \subset G_{0}^{F_{\bullet}} \mathrm{FL}_{S S}^{\text {loc }} M
$$

Proof. This is a direct consequence of the definition in formula (22), taking into account Lemma 3.19 and the fact that the filtered morphism $\phi$ induces an isomorphism of $\mathcal{D}_{\mathbb{A}_{z}^{1} \times{ }^{\prime}}$-modules by applying the functor $\mathrm{FL}_{S}^{\mathrm{loc}}$. by

From now on, we will specify the above situation to our main example, where the matrix $A$ is given

$$
A=\left(\begin{array}{cccccc}
1 & -1 & 0 & 0 & \ldots & 0 \\
1 & 0 & -1 & 0 & \ldots & 0 \\
\vdots & \ldots & & & & \\
1 & 0 & 0 & 0 & \ldots & -1
\end{array}\right)
$$

In particular, we have $d=n-1$, and $' S=\mathbb{G}_{m, t}$. We still write $M_{\dagger+}=\mathcal{H}^{0} p_{2,+} l_{\dagger+} \mathcal{O}_{S_{1} \times \mathbb{G}_{m, t}}^{\beta}$ and $M=\mathcal{H}^{0} \varphi_{+} \mathcal{O}_{S_{1} \times \mathbb{G}_{m, t}}^{\beta}$. We seek to improve the inclusion of the last corollary to an equality of lattices inside the $\mathcal{D}_{\mathbb{A}_{z}^{1} \times \mathbb{G}_{m, t}}$-modules $\mathrm{FL}_{\mathbb{G}_{m, t}}^{\text {loc }}\left(M_{\dagger+}\right) \cong \mathrm{FL}_{\mathbb{G}_{m, t}}^{\text {loc }}(M)$. We will follow an argument from the proof of [Sab08, Lem. 4.7]. In order to do so, we have to make more explicit the structure of the module $\mathrm{FL}_{\mathbb{G}_{m, t}}^{\text {loc }}(M) \cong \mathrm{FL}_{\mathbb{G}_{m, t}}^{\text {loc }}\left(M_{\dagger+}\right)$, which is done by the next lemma.

Lemma 3.23. (1) The singular locus $\Sigma:=\operatorname{Sing}(M)=\operatorname{Sing}\left(\mathcal{H}^{0} \varphi_{+} \mathcal{O}_{S_{1} \times \mathbb{G}_{m, t}}^{\beta}\right)$ is given by

$$
\Sigma=\bigcup_{\xi \in \mu_{n}}\left\{\left(n \cdot \xi \cdot t^{\prime}, t\right)\right\} \subset \mathbb{A}_{\lambda_{0}}^{1} \times \mathbb{G}_{m, t},
$$

where $t^{\prime}$ is an $n$-th root of $t$, chosen without loss of generality.

(2) Write $D:=\{0\} \times \mathbb{G}_{m, t} \subset \mathbb{A}_{z}^{1} \times \mathbb{G}_{m, t}$. Then $\mathrm{FL}_{\mathbb{G}_{m, t}}^{\text {loc }}(M)$ is $\mathcal{O}_{\mathbb{A}_{z}^{1} \times \mathbb{G}_{m, t}}(* D)$-locally free of rank $n$.

(3) Consider the sheaf $\widehat{\mathcal{O}}_{\mathbb{A}_{z}^{1} \times \mathbb{G}_{m, t}}$, which is the formal completion of $\mathcal{O}_{\mathbb{A}_{z}^{1} \times \mathbb{G}_{m, t}}$ along the divisor $\{0\} \times \mathbb{G}_{m, t}$. Then we have a decomposition (as sheaves on $\{0\} \times \mathbb{G}_{m, t}$ )

$$
\mathrm{FL}_{\mathbb{G}_{m, t}}^{\text {loc }}(M) \otimes_{\mathcal{O}_{\mathbb{A}_{z}^{1} \times \mathbb{G}_{m, t}}(* D)} \widehat{\mathcal{O}}_{\mathbb{A}_{z}^{1} \times \mathbb{G}_{m, t}}(* D) \cong \bigoplus_{\xi \in \mu_{n}} \widehat{\mathcal{E}}_{\xi} \otimes \widehat{\mathcal{N}}_{\alpha_{\xi}},
$$

where $\widehat{\mathcal{E}}_{\xi}:=\left(\widehat{\mathcal{O}}_{\mathbb{A}_{z}^{1} \times \mathbb{G}_{m, t}}(* D), d-d\left(n \cdot \xi \cdot t^{\prime} / z\right)\right)$ and $\widehat{\mathcal{N}}_{\alpha_{\xi}}=\left(\widehat{\mathcal{O}}_{\mathbb{A}_{z}^{1} \times \mathbb{G}_{m, t}}(* D), d+\alpha_{\xi} d z / z\right)$, with $\alpha_{\xi} \in \mathbb{C}$.

Proof. (1) It is well known that the singular locus of a Gauss-Manin system, i.e., of the topcohomology of the direct image complex $\varphi_{+} \mathcal{M}$, is nothing but the discriminant of the morphism $\varphi$ provided that the module $\mathcal{M}$ is smooth (which is the case here, since $\mathcal{M}=\mathcal{O}_{S_{1} \times \mathbb{G}_{m, t}}^{\beta}$ ). Recall (see formula (17) ) that ${ }^{\prime} \varphi\left(y_{1}, \ldots, y_{n-1}, t\right)=\left(\frac{1}{y_{1}}+\ldots+\frac{1}{y_{n-1}}+t \cdot y_{1} \cdot \ldots \cdot y_{n-1}, t\right)$. One easily 
checks (see, e.g., [DS04, §1.B.]) that a point $\left(y_{1}, \ldots, y_{n-1}\right) \in S_{1}$ is critical if and only if $y_{1}=\ldots=y_{n-1}=: y$ and $y^{n} \cdot t=1$ (and that all critical points are Morse). Then the critical values are as indicated.

(2) This is a direct consequence of the second point of [DS03, Thm. 1.11], since by the discussion above the singular locus $\Sigma$ satisfies the assumption (NC) of loc. cit. It is also known that the rank of $\mathrm{FL}_{\mathbb{G}_{m, t}}^{\text {loc }}(M)$ equals the global Milnor number of $\varphi$, i.e., the numbers of critical points, which is $n$.

(3) This follows from [Sab02, Ch. III, Thm. 5.7], since the critical values of $\varphi$, i.e., the eigenvalues of the pole part of $z^{2} \nabla_{z}$, are distinct for any $t \in \mathbb{G}_{m, t}$.

With these preparations, we can state the next result. As has been explained at the end of section 2 from page 7 on, it is the main step to show that the $\mathcal{R}_{\mathbb{A}_{z}^{1} \times \mathbb{G}_{m, t}}^{\text {int }}$-module $\widehat{\mathcal{H}}$ underlies a mixed Hodge module (that is, the content of Theorem 2.13). The explicit description of $\widehat{\mathcal{H}}$ as a cyclic quotient by two operators can be rather easily identified with the object $G_{0}^{F \bullet} \mathrm{FL}_{\mathbb{G}_{m, t}}^{\text {loc }} M$, as we will see below in the proof of Theorem 2.13, whereas the Hodge theoretic property we want (i.e., the fact that it is an object in $\left.\operatorname{IrmMHM}\left(\mathbb{G}_{m, t}\right)\right)$ holds for $G_{0}^{F_{\bullet}^{H}}{ }^{H_{s h}} \mathrm{FL}_{\mathbb{G}_{m, t}}^{\text {loc }} M_{\dagger++}$. Hence we need to identify these two $\mathcal{R}_{\mathbb{A}_{z}^{1} \times \mathbb{G}_{m, t}}^{\text {int }}$-modules.

Theorem 3.24. In the above situation, we have

$$
G_{0}^{F^{H_{s h}}} \mathrm{FL}_{\mathbb{G}_{m, t}}^{\mathrm{loc}} M_{\dagger+}=G_{0}^{F \bullet} \mathrm{FL}_{\mathbb{G}_{m, t}}^{\text {loc }} M
$$

Proof. We have already proved the inclusion

$$
G_{0}^{F^{H_{s h}}} \mathrm{FL}_{\mathbb{G}_{m, t}}^{\mathrm{loc}} M_{\dagger+} \subset G_{0}^{F \bullet} \mathrm{FL}_{\mathbb{G}_{m, t}}^{\text {loc }} M
$$

of $\mathcal{O}_{\mathbb{A}_{z}^{1} \times \mathbb{G}_{m, t}}$-modules. Since both sheaves coincide outside the divisor $D=\{0\} \times \mathbb{G}_{m, t}$, and since $\widehat{\mathcal{O}}_{\mathbb{A}_{z}^{1} \times \mathbb{G}_{m, t}}$ is $\mathcal{O}_{\left(\mathbb{A}_{z}^{1} \times \mathbb{G}_{m, t}, D\right)}$-flat, it is therefore sufficient to show that

$$
G_{0}^{F^{H_{s h}}} \mathrm{FL}_{\mathbb{G}_{m, t}}^{\text {loc }} M_{\dagger+} \otimes \widehat{\mathcal{O}}_{\mathbb{A}_{z}^{1} \times \mathbb{G}_{m, t}}=G_{0}^{F} \cdot \mathrm{FL}_{\mathbb{G}_{m, t}}^{\text {loc }} M \otimes \widehat{\mathcal{O}}_{\mathbb{A}_{z}^{1} \times \mathbb{G}_{m, t}}
$$

This follows as in the proof of [Sab08, Lem. 4.7]: Using the formal decomposition result from the last Lemma, both modules can be interpreted as microlocal filtered direct images under ' $p_{2}$ of two modules which coincide on ' $P$. In these direct images, the contributions from ' $P \backslash^{\prime} P^{*}$ vanish by the last statement of Proposition 3.16 and Lemma 3.9 (notice that inside $P=\mathbb{P}^{n} \times \mathbb{A}_{\lambda_{0}}^{1} \times S_{2}$, we have ${ }^{\prime} P^{*} \cap \Gamma X \cong S_{1} \times{ }^{\prime} S$ where we see ' $S$ as a subspace of $S_{2}$ via the embedding $\left.\iota\right)$, and therefore both modules are equal.

We are finally able to complete the proof of Theorem 2.13. It remains to show that the $\mathcal{R}_{\mathbb{A}_{z}^{1} \times \mathbb{G}_{m, t}}^{\text {int }}$ module

$$
\widehat{\mathcal{H}}=\frac{\mathcal{R}_{\mathbb{A}_{z}^{1} \times \mathbb{G}_{m, t}}^{\mathrm{int}}}{\left.\left(z^{2} \partial_{z}+n t z \partial_{t}-z \sum_{i=1}^{n} \alpha_{i}\right), \prod_{i=1}^{n} z\left(t \partial_{t}-\alpha_{i}\right)-t\right)}
$$

associated to the purely irregular hypergeometric $\mathcal{D}_{\mathbb{G}_{m, t}}$-module $\mathcal{H}\left(\alpha_{i}, \emptyset\right)$ underlies an object of the category $\operatorname{IrrMHM}\left(\mathbb{G}_{m, t}\right)$.

End of the proof of theorem 2.13. Let us assume first that $\alpha_{1}=0$, denote by $\alpha$ the vector $\left(\alpha_{2}, \ldots, \alpha_{n}\right)$ and put $\alpha_{0}:=0$. From Lemma 2.12 and Proposition 3.8 we conclude that

$$
\begin{aligned}
\widehat{\mathcal{H}} & \cong \iota^{+} \mathcal{H}^{0}\left(\pi_{2, *} \Omega_{S_{1} \times S_{2} / S_{2}}^{\bullet+d}[z], z(d-\kappa(\alpha) \wedge)-d f \wedge\right) \\
& \cong \mathcal{H}^{0}\left(\pi_{2, *} \Omega_{S_{1} \times \mathbb{G}_{m, t} / \mathbb{G}_{m, t}}^{\bullet d}[z], z(d-\kappa(\alpha) \wedge)-d^{\prime} f \wedge\right),
\end{aligned}
$$

recall that ' $f$ is the first component of ' $\varphi$, as written in $(17)$. Finally, it is easy to see from Definition 3.20 that we have

$$
\mathcal{H}^{0}\left(\pi_{2, *} \Omega_{S_{1} \times \mathbb{G}_{m, t} / \mathbb{G}_{m, t}}^{\bullet+d}[z], z(d-\kappa(\alpha) \wedge)-d^{\prime} f \wedge\right) \cong G_{0}^{F} \cdot \mathrm{FL}_{\mathbb{G}_{m, t}}^{\text {loc }} \mathcal{H}^{0 \prime} \varphi_{+} \mathcal{O}_{S_{1} \times \mathbb{G}_{m, t}}^{\alpha}=G_{0}^{F} \mathrm{FL}_{\mathbb{G}_{m, t}}^{\text {loc }} M .
$$


Since by Theorem 3.24 we can further conclude

$$
\widehat{\mathcal{H}} \cong G_{0}^{F_{\bullet}^{H_{s h}}} \mathrm{FL}_{\mathbb{G}_{m, t}}^{\text {loc }} M_{\dagger+},
$$

we obtain that $\widehat{\mathcal{H}}$ underlies an element of $\operatorname{IrMHM}\left(\mathbb{G}_{m, t}\right)$ by Lemma 3.21 (recall that $M_{\dagger+}$ underlies a pure polarizable complex Hodge module). Restricting $\widehat{\mathcal{H}}$ to $z=1$ we get the original $\mathcal{D}_{\mathbb{G}_{m, t}}$-module $\mathcal{H}\left(\alpha_{i} ; \emptyset\right)$.

Assume now that $\alpha_{1} \neq 0$. The tensor product of $\mathcal{R}_{\mathbb{A}_{z}^{1} \times \mathbb{G}_{m, t}}^{\text {int }}$-modules $\widehat{\mathcal{H}} \otimes \mathcal{O}_{\mathbb{A}_{z}^{1} \times \mathbb{G}_{m, t}} \widehat{\mathcal{K}}_{-\alpha_{1}}$ gives rise to the corresponding tensor product of twistor $\mathcal{D}$-modules on $\mathbb{G}_{m, t}$. This product can be presented as $\widehat{\mathcal{H}}\left(\alpha_{i}^{\prime} ; \emptyset\right)$, where $\alpha_{i}^{\prime}=\alpha_{i}-\alpha_{1}$ for every $i$. Reasoning as above, since $\alpha_{1}^{\prime}=0$, such tensor product is an irregular mixed Hodge module. Since $\widehat{\mathcal{K}}_{\alpha_{1}}$ is the faithful image of a mixed Hodge module on $\mathbb{G}_{m, t}$, the tensor product with it preserves the condition of being in $\operatorname{IrrMHM}\left(\mathbb{G}_{m, t}\right)$ due to [Sab18a, Cor. 0.5], and so is the case of our original $\mathcal{R}_{\mathbb{A}_{z}^{1} \times \mathbb{G}_{m, t}}^{\mathrm{int}}$-module

$$
\widehat{\mathcal{H}} \cong \widehat{\mathcal{H}}\left(\alpha_{i}^{\prime} ; \emptyset\right) \otimes_{\mathcal{O}_{\mathbb{A}_{z}^{1} \times \mathrm{G}_{m, t}}} \widehat{\mathcal{K}}_{\alpha_{1}} .
$$

\section{The irregular Hodge filtration}

In this section we will prove the second main result of this paper. Let us recall the notations used above. For a positive integer number $n$, and $\alpha_{1}, \ldots, \alpha_{n}$ real numbers, we consider the hypergeometric $\mathcal{D}_{\mathbb{G}_{m, t}}$-module $\mathcal{H}=\mathcal{H}\left(\alpha_{i} ; \emptyset\right)$ and its associated twistor $\mathcal{D}$-module $\widehat{\mathcal{H}}$ on $\mathbb{G}_{m, t}$ (we will denote by the same symbol the underlying hypergeometric $\mathcal{R}_{\mathbb{A}_{z}^{1} \times \mathbb{G}_{m, t}}^{\text {int }}$-module). From [Sab18a, Thm. 0.7] and Theorem 2.13 we know that there exists a unique irregular Hodge filtration of $\mathcal{H}$. We provide it in Theorem 4.7 below.

Let $\widehat{\mathcal{M}}$ a twistor $\mathcal{D}$-module on $X$, and call its associated $\mathcal{R}_{\mathbb{A}_{z}^{1} \times X}$-module the same way. If the $\mathcal{R}$-module $\widehat{\mathcal{M}}$ is integrable, good and well-rescalable (Sab18a, Def. 2.19]), we can define the irregular Hodge filtration of the underlying $\mathcal{D}_{X}$-module (say $\mathcal{M}$ ) following [ibid., Def. 2.22].

In our particular context, let us use the following notation (cf. [Sab18a, Not. 2.1]) for the sake of brevity: We will write $\mathcal{X}:=\mathbb{A}_{z}^{1} \times \mathbb{G}_{m, t},{ }^{\theta} \mathcal{X}=\mathcal{X} \times \mathbb{G}_{m, \theta},{ }^{\tau} \mathcal{X}=\mathcal{X} \times \mathbb{A}_{\tau}^{1}$ and ${ }^{\tau} \mathcal{X}_{0}=\mathcal{X} \times\{\tau=0\}$, where $\theta=1 / \tau$.

Let us summarize the process we follow to achieve our goal. We must first consider the rescaling of $\widehat{\mathcal{H}}$ : this is the inverse image ${ }^{\theta} \widehat{\mathcal{H}}:=\mu^{*} \mathcal{H}$ (as $\mathcal{O}_{\theta \mathcal{X}}$-module), endowed with a natural action of $\mathcal{R}_{\theta \mathcal{X}}^{\text {int }}$ as depicted in SSab18a, 2.4] (note that $\theta=\tau^{-1}$ ), where $\mu$ is the morphism given in [ibid., Not. 2.1] by

$$
\mu: \begin{aligned}
\theta \mathcal{X} & \rightarrow \mathcal{X} \\
(z, t, \theta) & \mapsto(z \theta, t) .
\end{aligned}
$$

This is done right below. Then we have to invert $\theta$ to obtain an $\mathcal{R}_{\tau \mathcal{X}}^{\text {int }}\left(*^{\tau} \mathcal{X}_{0}\right)$-module ${ }^{\tau} \widehat{\mathcal{H}}$, to work in the context of [Sab18a, §2.3]. Finally, the irregular Hodge filtration is obtained from a suitable $V$-filtration along the divisor $\tau=0$ defined on ${ }^{\tau} \widehat{\mathcal{H}}$, which is called ${ }^{\tau} V$-filtration (the new symbol ${ }^{\tau} V$ is to make clear the variety over which we are working; note the same convention in [ibid.], from Remark 2.20 on). We will actually define a filtration on ${ }^{\tau} \widehat{\mathcal{H}}$ in Definition 4.4 , and then prove that it equals the $\tau$-filtration in Proposition 4.6, following [Moc15a, §2.1.2].

Proposition 4.1. Recall that we could write $\widehat{\mathcal{H}}$ as the $\mathcal{R}_{\mathcal{X}}^{\text {int }}$-module $\mathcal{R}_{\mathcal{X}}^{\text {int }} /(P, H)$, where $P$ and $H$ were, respectively,

$$
z^{2} \partial_{z}+n z t \partial_{t}+\gamma z \text { and } \prod_{i=1}^{n} z\left(t \partial_{t}-\alpha_{i}\right)-t,
$$

for certain value of $\gamma$. Then, ${ }^{\theta} \widehat{\mathcal{H}}=\mathcal{R}_{\theta \mathcal{X}}^{\text {int }} /\left(P,{ }^{\theta} R,{ }^{\theta} H\right)$, with $P$ as before and

$$
{ }^{\theta} R=z^{2} \partial_{z}-z \theta \partial_{\theta} \text { and }{ }^{\theta} H=\prod_{i=1}^{n} z \theta\left(t \partial_{t}-\alpha_{i}\right)-t .
$$


Proof. The morphism $\mu$ can be decomposed as $p \circ \phi$, where $p$ is the canonical projection from ${ }^{\theta} \mathcal{X}$ to $\mathcal{X}$ and $\phi$ is the automorphism of ${ }^{\theta} \mathcal{X}$ given by $(z, t, \theta) \mapsto(z \theta, t, \theta)$. Then, in the category of $\mathcal{O}_{\theta \mathcal{X}}$-modules we have that

$$
\mu^{*} \widehat{\mathcal{H}} \cong \phi^{*} p^{*} \widehat{\mathcal{H}} \cong \phi^{*} \mathcal{R}_{\theta \mathcal{X}}^{\text {int }} /\left(z \theta \partial_{\theta}, P, H\left(z, t, \partial_{t}\right)\right) \cong \mathcal{R}_{\theta \mathcal{X}}^{\text {int }} /\left(z^{2} \partial_{z}-z \theta \partial_{\theta}, P, H\left(z \theta, t, \partial_{t}\right)\right),
$$

by the chain rule.

What remains now is to prove the compatibilities of [Sab18a, 2.4] among the actions of $\mathcal{R}_{\theta \mathcal{X}}^{\text {int }}$ on $\mathcal{R}_{\theta \mathcal{X}}^{\text {int }} /\left(P,{ }^{\theta} R,{ }^{\theta} H\right)$, seen as $\mu^{*} \widehat{\mathcal{H}}=\mu^{-1} \widehat{\mathcal{H}} \otimes_{\mu^{-1}} \mathcal{O}_{\mathcal{X}} \mathcal{O}_{\theta \mathcal{X}}$. They are just a consequence of the presence of $z^{2} \partial_{z}-z \theta \partial_{\theta}$ in the ideal with which we take the quotient in $\mu^{*} \widehat{\mathcal{H}}$ and how $z, z \partial_{i}$ or $z^{2} \partial_{z}$ act on both factors of the tensor product. For instance, if we multiply by $z$ at the right-hand one is the same as if we multiply by $z \theta$ at the left-hand one.

Remark 4.2. Let $i_{\tau=z}$ be the inclusion $\mathbb{G}_{m, z} \times \mathbb{G}_{m, t} \hookrightarrow^{\theta} \mathcal{X}$ given by $(z, t) \mapsto(z, t, \tau)$. Note that, according to the fourth point of Sab18a, Lem. 2.5], we must have $i_{\tau=z}^{*} \theta \widehat{\mathcal{H}} \cong \pi^{0,+} \mathcal{H}$ as $\mathcal{R}_{\mathbb{G}_{m, z} \times \mathbb{G}_{m, t^{-}}}$ modules, with $\pi^{0}$ being the projection $\mathbb{G}_{m, z} \times \mathbb{G}_{m, t} \rightarrow \mathbb{G}_{m, t}$. Indeed, we have

$$
\begin{aligned}
i_{\tau=z}^{*}{ }^{\theta} \widehat{\mathcal{H}}=\mathcal{R}_{\theta \mathcal{X}}^{\text {int }} /\left(P,{ }^{\theta} R,{ }^{\theta} H, \theta z-1\right) & \cong \mathcal{O}_{\mathbb{G}_{m, \theta} \times \mathbb{A}_{z}^{1} \times \mathbb{G}_{m, t}}\left\langle z t \partial_{t}\right\rangle /\left(H_{1}, \theta z-1\right) \\
& \cong \mathcal{O}_{\mathbb{G}_{m, \theta} \times \mathbb{G}_{m, z} \times \mathbb{G}_{m, t}}\left\langle z t \partial_{t}\right\rangle /\left(H_{1}, \theta-z^{-1}\right) \\
& \cong \mathcal{O}_{\mathbb{G}_{m, z} \times \mathbb{G}_{m, t}}\left\langle z t \partial_{t}\right\rangle /\left(H_{1}\right) \\
& \cong \mathcal{O}_{\mathbb{G}_{m, z} \times \mathbb{G}_{m, t}} \otimes_{\mathcal{O}_{\mathbb{G}_{m, t}}} \mathcal{H} \cong \pi^{0,+} \mathcal{H},
\end{aligned}
$$

where $H_{1}$ is the result of replacing $z$ by 1 at the expression for $H$.

As said above, in order to continue, we must pass from ${ }^{\theta} \mathcal{X}$ to ${ }^{\tau} \mathcal{X}$. Therefore, we invert $\theta$ and extend $\tau$ to the affine line to get a $\mathcal{R}_{\tau \mathcal{X}}^{\text {int }}\left(*^{\tau} \mathcal{X}_{0}\right)$-module. In other words, call inv : $\mathbb{G}_{m, \theta} \rightarrow \mathbb{G}_{m, \tau}$ the inversion operator $\theta \mapsto \theta^{-1}=\tau$ and $j: \mathbb{G}_{m, \tau} \hookrightarrow \mathbb{A}_{\tau}^{1}$ the canonical inclusion. From now on, we will denote by ${ }^{\tau} \widehat{\mathcal{H}}$ the $\mathcal{R}_{\tau \mathcal{X}}^{\text {int }}\left(*^{\tau} \mathcal{X}_{0}\right)$-module $\left(\mathrm{id}_{\mathcal{X}} \times(j \circ \mathrm{inv})\right)_{*}{ }^{\theta} \widehat{\mathcal{H}}$. By virtue of Proposition 4.1 we can write ${ }^{\tau} \widehat{\mathcal{H}}$ as the $\mathcal{R}_{\tau}^{\text {int }}\left(*^{\tau} \mathcal{X}_{0}\right)$-module ${ }^{\tau} \widehat{\mathcal{H}}=\mathcal{R}_{\tau}^{\text {int }}\left(*^{\tau} \mathcal{X}_{0}\right) /\left(P,{ }^{\tau} R,{ }^{\tau} H\right)$, with $P$ as before and

$$
{ }^{\tau} R=z^{2} \partial_{z}+z \tau \partial_{\tau} \text { and }{ }^{\tau} H=\prod_{i=1}^{n} \frac{z}{\tau}\left(t \partial_{t}-\alpha_{i}\right)-t .
$$

Lemma 4.3. For each $k=0, \ldots, n-1$, let $Q_{k}$ be the operator

$$
Q_{k}=(-n)^{k} \prod_{j=1}^{k} \frac{z}{\tau}\left(t \partial_{t}-\alpha_{j}\right),
$$

where the empty product must be understood as one. Then the $Q_{k}$ form a basis of $\widehat{\mathcal{H}}$ as an $\mathcal{O}_{\tau} \mathcal{X}\left(*^{\tau} \mathcal{X}_{0}\right)$ module. The integrable connection arising from the $\mathcal{R}_{\tau \mathcal{X}}^{\text {int }}\left(*^{\tau} \mathcal{X}_{0}\right)$-module structure associated with ${ }^{\tau} \widehat{\mathcal{H}}$ has the following matrix expression with respect to that basis:

$$
\nabla \underline{Q}=\underline{Q}\left(\left(\tau A_{0}+z A_{\infty}\right) \frac{d z}{z^{2}}+\left(-\tau A_{0}+z A_{\infty}^{\prime}\right) \frac{d t}{n z t}-\left(\tau A_{0}+z A_{\infty}\right) \frac{d \tau}{z \tau}\right),
$$

where $A_{0}, A_{\infty}^{\prime}$ and $A_{\infty}$ are the matrices

$$
\begin{gathered}
A_{0}=\left(\begin{array}{cccc}
0 & & & (-n)^{n} t \\
1 & \ddots & & 0 \\
& \ddots & 0 & \vdots \\
& & 1 & 0
\end{array}\right), A_{\infty}^{\prime}=\operatorname{diag}\left(n \alpha_{1}, \ldots, n \alpha_{n}\right) \\
\quad \text { and } A_{\infty}=\operatorname{diag}(0,1, \ldots, n-1)-\gamma I_{n}-A_{\infty}^{\prime} .
\end{gathered}
$$


Proof. We can use the expressions for ${ }^{\tau} R$ and $P$ to replace the classes of $z \tau \partial_{\tau}$ and $z^{2} \partial_{z}$, respectively, in terms of $z t \partial_{t}$. If we extend for a moment the definition of the $Q_{k}$ for values of $k$ greater than $n-1$ taking $Q_{k}:=\left(z \tau^{-1} t \partial_{t}\right)^{k-n+1} Q_{n-1}$, we see that ${ }^{\tau} \widehat{\mathcal{H}}$ is generated as a $\mathcal{O}_{\tau} \mathcal{X}\left(*^{\tau} \mathcal{X}_{0}\right)$-module by the $Q_{k}$, for $k \geq 0$ (since it is obviously generated by the powers of $z t \partial_{t}$, and those can be expressed by the $Q_{k}$ ). If we focus now at the degree in $z t \partial_{t}$ of the generators, we can use ${ }^{\tau} H$ to get rid of any $Q_{k}$ with $k \geq n$. Since $\operatorname{deg}_{z t \partial_{t}} Q_{k}=k$, they must be linearly independent over $\mathcal{O}_{\tau} \mathcal{X}\left(*^{\tau} \mathcal{X}_{0}\right)$ and so they are a basis.

Let now $k<n-1$. Then from the relation $-n z / \tau\left(t \partial_{t}-\alpha_{k+1}\right) Q_{k}=Q_{k+1}$ we can write that $n z t \partial_{t} Q_{k}=-\tau Q_{k+1}+n z \alpha_{k+1} Q_{k}$. Now if $k=n-1$, then

$$
-n z / \tau\left(t \partial_{t}-\alpha_{n}\right) Q_{n-1}=(-n)^{n} \prod_{j=1}^{n} z / \tau\left(t \partial_{t}-\alpha_{j}\right)=(-n)^{n} t .
$$

This gives us the second summand of the formula above in the statement.

The first one is a consequence of the last one and the fact that the class of $z^{2} \partial_{z}+z \tau \partial_{\tau}$ vanishes in $\tau \widehat{\mathcal{H}}$; let us show the expression for the latter.

Take again $k<n-1$. Then,

$$
\begin{gathered}
z \tau \partial_{\tau} Q_{k}=(-n)^{k} z \tau \partial_{\tau} \tau^{-k} \prod_{j=1}^{k} z\left(t \partial_{t}-\alpha_{j}\right)=(-n)^{k} z\left(-k \tau^{-k}+\tau^{-k+1} \partial_{\tau}\right) \prod_{j=1}^{k} z\left(t \partial_{t}-\alpha_{j}\right)= \\
=-k z Q_{k}+Q_{k} z \tau \partial_{\tau}=-k z Q_{k}+Q_{k} z\left(n t \partial_{t}+\gamma\right)=z\left(n t \partial_{t}+\gamma-k\right) Q_{k}=-\tau Q_{k+1}+z\left(n \alpha_{k+1}+\gamma-k\right) Q_{k} .
\end{gathered}
$$

The analogous calculation for $k=n-1$ gives us that $z \tau \partial_{\tau} Q_{n-1}=-\tau(-n)^{n} t+z\left(n \alpha_{n}+\gamma-(n-\right.$ 1)) $Q_{n-1}$.

Definition 4.4. For each $\alpha \in \mathbb{R}$, let us define the following subsets of ${ }^{\tau} \widehat{\mathcal{H}}$ :

$$
\begin{aligned}
{ }^{\top}{ }_{\alpha}{ }^{\tau} \widehat{\mathcal{H}}: & =\left\{\sum_{k=0}^{n-1} f_{k} \tau^{\nu_{k}} Q_{k}: f_{k} \in \mathcal{O}_{\tau \mathcal{X}}, \max \left(k-n \alpha_{k+1}-\gamma-\nu_{k}\right) \leq \alpha\right\}, \\
{ }^{\tau}{ }_{<\alpha}{ }^{\tau} \widehat{\mathcal{H}}: & =\left\{\sum_{k=0}^{n-1} f_{k} \tau^{\nu_{k}} Q_{k}: f_{k} \in \mathcal{O}_{\tau \mathcal{X}}, \max \left(k-n \alpha_{k+1}-\gamma-\nu_{k}\right)<\alpha\right\} .
\end{aligned}
$$

Remark 4.5. Note that the ${ }^{\tau} U_{\alpha}^{\tau} \widehat{\mathcal{H}}$ form an increasing filtration of ${ }^{\tau} \widehat{\mathcal{H}}$, indexed by the real numbers but with a discrete set of jumping numbers, such that $\tau^{\tau} U_{\alpha}{ }^{\tau} \widehat{\mathcal{H}}={ }^{\tau} U_{\alpha-1}{ }^{\tau} \widehat{\mathcal{H}}$ for any $\alpha$. The graded piece associated with $\alpha$ is $\operatorname{Gr}_{\alpha}^{\tau} U \widehat{\mathcal{H}}={ }^{\tau} U_{\alpha}^{\tau} \widehat{\mathcal{H}} /{ }^{\tau} U_{<\alpha}{ }^{\tau} \widehat{\mathcal{H}}$.

In the definition of the ${ }^{\tau} U_{\alpha}{ }^{\tau} \widehat{\mathcal{H}}$ all the exponents $\nu_{k}$ of the powers of $\tau$ accompanying the $f_{k} Q_{k}$ satisfy that $\nu_{k} \geq-\alpha+k-n \alpha_{k+1}-\gamma$. Therefore, we can define the steps of the filtration in an alternative way, as the free $\mathcal{O}_{\tau \mathcal{X}}$-modules of finite rank

$$
\tau_{\alpha}{ }^{\tau} \widehat{\mathcal{H}}=\bigoplus_{k=0}^{n-1} \mathcal{O}_{\tau \mathcal{X}} \cdot \tau^{\nu_{\alpha}(k)} Q_{k}
$$

where $\nu_{\alpha}(k)=\left\lceil-\alpha+k-\gamma-n \alpha_{k+1}\right\rceil$.

With this expression it is easy to see that ${ }^{\tau} U_{\alpha}^{\tau} \widehat{\mathcal{H}} /(\tau-z)^{\tau} U_{\alpha}{ }^{\tau} \widehat{\mathcal{H}}$ is the $z$-graded free $\mathcal{O}_{\mathcal{X}}$-module $\bigoplus_{k} \mathcal{O}_{\mathcal{X}} z^{\nu_{\alpha}(k)} \bar{Q}_{k}$, where

$$
\bar{Q}_{k}=(-n)^{k} \prod_{j=1}^{k}\left(t \partial_{t}-\alpha_{j}\right)
$$

and that the graded pieces $\mathrm{Gr}_{\alpha}^{\tau} \mathcal{T}^{\tau} \widehat{\mathcal{H}}$ are

$$
\operatorname{Gr}_{\alpha}^{\tau} U \tau \widehat{\mathcal{H}}=\bigoplus_{k=0}^{n-1} \mathcal{O}_{\mathcal{X}} \cdot \tau^{\nu_{\alpha}(k)} Q_{k}
$$

which are strict $\mathcal{R}_{\mathcal{X}}$-modules. 
Recall that as in the case of $\mathcal{D}$-modules, we have a notion of strict specializability and $V$-filtration for

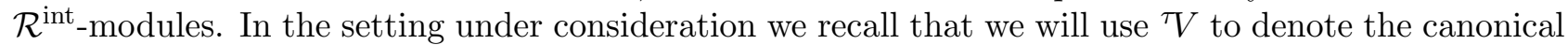
$V$-filtration of a $\mathcal{R}_{\tau \mathcal{X}}^{\text {int }}$-module. We recall as well the reference Moc15a, §2.1.2] for more details.

Proposition 4.6. Assume the $\alpha_{i}$ lie in the interval $[0,1)$, increasingly ordered. Then, $\widehat{\mathcal{H}}$ is strictly $\mathbb{R}$-specializable along ${ }^{\tau} \mathcal{X}_{0}$ and its ${ }^{\tau} V$-filtration is in fact given by the ${ }^{\tau} U_{\alpha}{ }^{\tau} \widehat{\mathcal{H}}$.

Proof. First of all we will see that ${ }^{\tau} U_{\alpha}{ }^{\tau} \widehat{\mathcal{H}}$ is the ${ }^{\tau} V$-filtration of ${ }^{\tau} \widehat{\mathcal{H}}$, following Moc15a, $\left.\S \S 2.1 .2 .1,2.1 .2 .2\right]$. Apart from what we already shown at the remark above, what remains then is showing conditions iii' and $\mathrm{v}$ of [ibid., $\S 2.1 .2]$ and prove that the ${ }^{\tau} U_{\alpha}{ }^{\tau} \widehat{\mathcal{H}}$ are coherent $V_{0} \mathcal{R}_{\mathcal{X}}$-modules. We will start by the second condition. We will consider then the mappings $\mathfrak{p}, \mathfrak{e}$ given by

$$
\begin{aligned}
(\mathfrak{p}, \mathfrak{e}): \mathbb{R} \times \mathbb{C} & \longrightarrow \mathbb{R} \times \mathbb{C} \\
(\beta, \omega) & \longmapsto\left(\beta+2 \Re(z \bar{\omega}),-\beta z+\omega-\bar{\omega} z^{2}\right) .
\end{aligned}
$$

We ought to see now that the operator $z \tau \partial_{\tau}-\mathfrak{e}(\beta, \omega)$ is nilpotent on the graded pieces $\operatorname{Gr}_{\alpha}^{\tau} \tau^{\tau} \widehat{\mathcal{H}}$ only for a finite amount of $(\beta, \omega) \in \mathcal{K}:=\left\{\beta+2 \Re\left(z_{0} \bar{\omega}\right)=\alpha\right\}$, for any value $z_{0}$ of $z$. Moreover, ${ }^{\tau} \widehat{\mathcal{H}}$ will be strictly $\mathbb{R}$-specializable if those $(\beta, \omega)$ belong in fact to $\mathbb{R} \times\{0\}$ (cf. [Sab18a, §1.3.a]).

Take then $(\beta, \omega) \in \mathcal{K}$ and $f \tau^{\nu} Q_{k} \in{ }^{\tau} U_{\alpha} \widehat{\mathcal{H}}$, with $f \in \mathcal{O}_{\tau \mathcal{X}}$. We must have that $k-n \alpha_{k+1}-\gamma-\nu \leq \alpha$. Assume that $k<n-1$. Thanks to Lemma 4.3 we know that

$$
\left(z \tau \partial_{\tau}-\mathfrak{e}(\beta, \omega)\right) f \tau^{\nu} Q_{k}=\left(z \tau \partial_{\tau}+\left(\nu+n \alpha_{k+1}+\gamma-k+\beta\right) z-\omega+\bar{\omega} z^{2}\right)(f) \tau^{\nu} Q_{k}-\tau^{\nu+1} Q_{k+1} .
$$

Recall that the $\alpha_{i}$ are increasingly ordered. Thus $f \tau^{\nu+1} Q_{k+1}$ lives in ${ }^{\tau} U_{\alpha} \tau \widehat{\mathcal{H}}$, for

$$
\left.k+1-n \alpha_{k+2}-\gamma-\nu-1 \leq\left((k+1)-n \alpha_{k+2}-\gamma\right)-\left(k-n \alpha_{k+1}-\gamma\right)\right)-1+\alpha \leq \alpha .
$$

Now we should look at what happens to the class of $f \tau^{\nu+1} Q_{k+1}$ in the $\alpha$-graded piece of $\tau \widehat{\mathcal{H}}$.

Note that $\left[f \tau^{\nu} Q_{k}\right] \neq 0$ if and only if $\nu+n \alpha_{k+1}+\gamma-k+\alpha=0$, so

$$
\begin{gathered}
\left(z \tau \partial_{\tau}-\mathfrak{e}(\beta, \omega)\right) f \tau^{\nu} Q_{k}=\left(z \tau \partial_{\tau}+(\beta-\alpha) z-\omega+\bar{\omega} z^{2}\right)(f) \tau^{\nu} Q_{k}-\tau^{\nu+1} Q_{k+1}= \\
=\left(z \tau \partial_{\tau}-2 \Re\left(z_{0} \bar{\omega}\right) z-\omega+\bar{\omega} z^{2}\right)(f) \tau^{\nu} Q_{k}-\tau^{\nu+1} Q_{k+1} .
\end{gathered}
$$

Now notice that $\tau$ divides $\tau \partial_{\tau}(f)$, so in fact $z \tau \partial_{\tau}(f) \tau^{\nu} Q_{k} \in{ }^{\tau} U_{\alpha-1}{ }^{\tau} \widehat{\mathcal{H}}$ and then we can further reduce our expression to

$$
\left(z \tau \partial_{\tau}-\mathfrak{e}(\beta, \omega)\right) f \tau^{\nu} Q_{k}=\left(-\omega-2 \Re\left(z_{0} \bar{\omega}\right) z+\bar{\omega} z^{2}\right) f \tau^{\nu} Q_{k}-\tau^{\nu+1} Q_{k+1} .
$$

On the other hand, $\tau^{\nu+1} Q_{k+1}$ does not vanish either in $\operatorname{Gr}_{\alpha}^{\tau} \tau \widehat{\mathcal{H}}$ if and only if $\alpha_{k+2}=\alpha_{k+1}$. Indeed, we know that $\nu+n \alpha_{k+1}+\gamma-k+\alpha=0$, so doing the same as before, $k+1-n \alpha_{k+2}-\gamma-\nu-1=$ $\alpha+n\left(\alpha_{k+2}-\alpha_{k+1}\right)$ and the claim follows. Furthermore, in order to $\left(z \tau \partial_{\tau}-\mathfrak{e}(\beta, \omega)\right)$ to vanish, we should impose that $\omega=0$, just by looking at the coefficients of the powers of $z$ in the expression for $f$.

Now if $k=n-1$, then everything would be the same as before except $-\tau^{\nu+1} Q_{k+1}$, which becomes $-(-n)^{n} t \tau^{\nu+1}$, whose class vanishes obviously in the graded piece under consideration.

In conclusion, $\left(z \tau \partial_{\tau}-\mathfrak{e}(\beta, \omega)\right)^{l} f \tau^{\nu} Q_{k}$ can only vanish in $\operatorname{Gr}_{\alpha}^{\tau} \tau^{\tau} \widehat{\mathcal{H}}$ if $\alpha=\beta$ (and then $\omega=0$ ), and does not do so until we get to an index $k+l$ such that $\alpha_{k+l}$ is strictly bigger than $\alpha_{k}$. Since there is a finite set of indexes, $\left(z \tau \partial_{\tau}+\alpha z\right)$ is nilpotent, of nilpotency index $n$ at most.

Condition iii' in Moc15a, $\$ 2.1 .2 .2$ ] is equivalent to $z \tau \partial_{\tau}{ }^{\top} U_{\alpha}{ }^{\tau} \widehat{\mathcal{H}} \subseteq{ }^{\tau} U_{\alpha}{ }^{\tau} \widehat{\mathcal{H}}$, using that ${ }^{\tau} U_{\alpha}{ }^{\tau} \widehat{\mathcal{H}}=$ $\tau^{\tau} U_{\alpha+1}{ }^{\tau} \hat{\mathcal{H}}$, and such claim is a consequence from an argument very similar to the proof of condition $\mathrm{v}$ above. Finally, since $V_{0} \mathcal{R}_{\mathcal{X}}=\mathcal{O}_{\tau \mathcal{X}}\left\langle z \partial_{t}, z \tau \partial_{\tau}\right\rangle$, it is clear from the computations above and the alternative expression for the filtration steps in Remark 4.5 that they are cyclic $V_{0} \mathcal{R}_{\mathcal{X}}$-modules, and then coherent.

Summing up and noting that all the calculation was in fact independent of $z_{0},{ }^{\tau} \widehat{\mathcal{H}}$ is strictly $\mathbb{R}$ specializable along ${ }^{\tau} \mathcal{X}_{0}$.

Finally, we are able to state and prove our main result. 
Theorem 4.7. Let as before $\alpha_{1}, \ldots, \alpha_{n}$ be real numbers in $[0,1)$, increasingly ordered, and put $\mathcal{H}=$ $\mathcal{H}\left(\alpha_{i}, \emptyset\right)$. For each $k=1, \ldots, n$, set $\rho(k)=-n \alpha_{k}+k$. Then the jumping numbers of the irregular Hodge filtration of $\mathcal{H}$ are, up to an overall real shift, the numbers $\rho(k)$. The irregular Hodge numbers are the multiplicities of those jumping numbers, or in other words, the nonzero values of $\left|\rho^{-1}(x)\right|$, for $x$ real.

Moreover, recall that for all $r=0, \ldots, n-1$, we had $\nu_{\alpha}(r)=\left\lceil-\alpha+r-\gamma-n \alpha_{r+1}\right\rceil$, and that the operators $\bar{Q}_{r}$ were defined as

$$
\bar{Q}_{r}=(-n)^{r} \prod_{i=1}^{r}\left(t \partial_{t}-\alpha_{i}\right) .
$$

Then, the irregular Hodge filtration $F_{\bullet}^{\mathrm{irr}} \mathcal{H}$ is given by

$$
F_{\alpha+j}^{\mathrm{irr}} \mathcal{H}=\bigoplus_{k: j \geq \nu_{\alpha}(k)} \mathcal{O}_{X} \bar{Q}_{k}
$$

Proof. Since we know that $\widehat{\mathcal{H}}$ underlies an object in $\operatorname{IrrMHM}\left(\mathbb{G}_{m, t}\right)$ by Theorem 2.13 , we conclude by Sab18a, Def. 2.52] that $\widehat{\mathcal{H}}$ is well-rescalable (as defined in [ibid., Def. 2.19]) and so we apply [ibid., Def. 2.22]. From Remark 4.5, we have

$$
i_{\tau=z}^{*} V_{\alpha}{ }^{\tau} \widehat{\mathcal{H}}={ }^{\tau} V_{\alpha}{ }^{\tau} \widehat{\mathcal{H}} /(\tau-z)^{\tau} V_{\alpha}{ }^{\tau} \widehat{\mathcal{H}}=\bigoplus_{k} \mathcal{O}_{\mathcal{X}} z^{\nu_{\alpha}(k)} \bar{Q}_{k},
$$

which is z-graded of finite rank.

Denote by $\pi$ the projection $\mathcal{X} \rightarrow X$. Then, the $z$-adic filtration on $\pi^{*} \mathcal{H}\left[z^{-1}\right]$ induces a filtration on $i_{\tau=z}^{*} V_{\alpha}^{\tau} \widehat{\mathcal{H}}$, given by

$$
F_{r} i_{\tau=z}^{*}{ }^{\tau} V_{\alpha}^{\tau} \widehat{\mathcal{H}}:=\bigoplus_{s \leq r}\left(\bigoplus_{k: s \geq \nu_{\alpha}(k)} \mathcal{O}_{X} \bar{Q}_{k}\right) z^{s} .
$$

Then, $\operatorname{Gr}^{F}\left(i_{\tau=z}^{*} V_{\alpha}^{\tau} \widehat{\mathcal{H}}\right)$ is the Rees module associated to a new good filtration $F_{\alpha+\bullet}^{\text {irr }}$ on $\mathcal{H}$, for some $k=0, \ldots, n-1$, which is the irregular Hodge filtration. More concretely, $F_{\bullet}^{\text {irr }} \mathcal{H}$ is given by

$$
F_{\alpha+j}^{\mathrm{irr}} \mathcal{H}=\bigoplus_{k: j \geq \nu_{\alpha}(k)} \mathcal{O}_{X} \bar{Q}_{k}
$$

Therefore, its jumping numbers are $-\gamma+i-1-n \alpha_{i}$ for $i=1, \ldots, n$. Since the irregular Hodge filtration is defined up to an overall real shift, we can normalize the jumping numbers to $i-n \alpha_{i}$ and the irregular Hodge numbers will be their multiplicities.

\section{REFERENCES}

[Ado94] Alan Adolphson, Hypergeometric functions and rings generated by monomials, Duke Math. J. 73 (1994), no. 2, 269-290.

[Ari10] D. Arinkin, Rigid irregular connections on $\mathbb{P}^{1}$, Compos. Math. 146 (2010), no. 5, 1323-1338.

[BH06] Lev A. Borisov and R. Paul Horja, Mellin-Barnes integrals as Fourier-Mukai transforms, Adv. Math. 207 (2006), no. 2, 876-927.

[BMW18] Christine Berkesch, Laura Felicia Matusevich, and Uli Walther, On normalized Horn systems, preprint arXiv:1806.03355 [math.AG], 2018.

[Bri70] Egbert Brieskorn, Die Monodromie der isolierten Singularitäten von Hyperflächen, Manuscripta Math. 2 (1970), 103-161.

[CDRS18] Alberto Castaño Domínguez, Thomas Reichelt, and Christian Sevenheck, Examples of hypergeometric twistor D-modules, preprint arXiv:1803.04886 [math.AG], to appear in Algebra Number Theory, 2018.

[CG11] Alessio Corti and Vasily Golyshev, Hypergeometric equations and weighted projective spaces, Sci. China Math. 54 (2011), no. 8, 1577-1590.

[CK99] David A. Cox and Sheldon Katz, Mirror symmetry and algebraic geometry, Mathematical Surveys and Monographs, vol. 68, American Mathematical Society, Providence, RI, 1999.

[DE03] Andrea D'Agnolo and Michael Eastwood, Radon and Fourier transforms for D-modules, Adv. Math. 180 (2003), no. 2, 452-485.

[Dim04] Alexandru Dimca, Sheaves in topology, Universitext, Springer-Verlag, Berlin, 2004.

[DL91] J. Denef and F. Loeser, Weights of exponential sums, intersection cohomology, and Newton polyhedra, Invent. Math. 106 (1991), no. 2, 275-294. 
[DS03] Antoine Douai and Claude Sabbah, Gauss-Manin systems, Brieskorn lattices and Frobenius structures. I, Ann. Inst. Fourier (Grenoble) 53 (2003), no. 4, 1055-1116.

[DS04] _ Gauss-Manin systems, Brieskorn lattices and Frobenius structures. II, Frobenius manifolds, Aspects Math., E36, Vieweg, Wiesbaden, 2004, pp. 1-18.

[DS13] Michael Dettweiler and Claude Sabbah, Hodge theory of the middle convolution, Publ. Res. Inst. Math. Sci. 49 (2013), no. 4, 761-800.

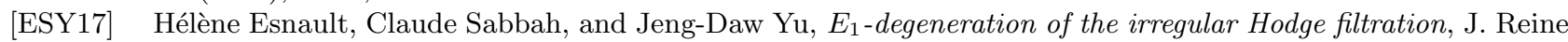
Angew. Math. 729 (2017), 171-227.

[Fed18] Roman Fedorov, Variations of Hodge structures for hypergeometric differential operators and parabolic Higgs bundles, Int. Math. Res. Not. IMRN (2018), no. 18, 5583-5608.

[GKZ94] Israel M. Gel'fand, Mikhail M. Kapranov, and Andrei V. Zelevinsky, Discriminants, resultants, and multidimensional determinants, Mathematics: Theory \& Applications, Birkhäuser Boston Inc., Boston, MA, 1994.

[GMS09] Ignacio de Gregorio, David Mond, and Christian Sevenheck, Linear free divisors and Frobenius manifolds, Compositio Mathematica 145 (2009), no. 5, 1305-1350.

[HS07] Claus Hertling and Christian Sevenheck, Nilpotent orbits of a generalization of Hodge structures., J. Reine Angew. Math. 609 (2007), 23-80.

[HTT08] Ryoshi Hotta, Kiyoshi Takeuchi, and Toshiyuki Tanisaki, D-modules, perverse sheaves, and representation theory, Progress in Mathematics, vol. 236, Birkhäuser Boston, Inc., Boston, MA, 2008, translated from the 1995 Japanese edition by Takeuchi.

[Kat90] Nicholas M. Katz, Exponential sums and differential equations, Annals of Mathematics Studies, vol. 124, Princeton University Press, Princeton, NJ, 1990.

[KKP17] Ludmil Katzarkov, Maxim Kontsevich, and Tony Pantev, Bogomolov-Tian-Todorov theorems for LandauGinzburg models, J. Differential Geom. 105 (2017), no. 1, 55-117.

[Kou76] Anatoli G. Kouchnirenko, Polyèdres de Newton et nombres de Milnor, Invent. Math. 32 (1976), no. 1, 1-31.

[Mar18] Nicolas Martin, Middle multiplicative convolution and hypergeometric equations, preprint arXiv:1809.08867 [math.AG], 2018.

[Moc11] Takuro Mochizuki, Wild harmonic bundles and wild pure twistor D-modules, Astérisque (2011), no. 340, $\mathrm{x}+607$.

[Moc15a] _ Mixed twistor D-modules, Lecture Notes in Mathematics, vol. 2125, Springer, Cham, 2015.

[Moc15b] _ Twistor property of GKZ-hypergeometric systems, preprint arXiv:1501.04146 [math.AG], 2015.

[Rei14] Thomas Reichelt, Laurent polynomials, GKZ-hypergeometric systems and mixed Hodge modules, Compositio Mathematica (150) (2014), 911-941.

[RS15a] Thomas Reichelt and Christian Sevenheck, Hypergeometric Hodge modules, preprint arXiv:1503.01004 [math.AG], to appear in Algebraic Geometry, 2015.

[RS15b]_ Logarithmic Frobenius manifolds, hypergeometric systems and quantum D-modules, Journal of Algebraic Geometry 24 (2015), no. 2, 201-281.

[RS17] _ Non-affine Landau-Ginzburg models and intersection cohomology, Ann. Sci. Éc. Norm. Supér. (4) 50 (2017), no. 3, 665-753.

[Sab02] Claude Sabbah, Déformations isomonodromiques et variétés de Frobenius, Savoirs Actuels, EDP Sciences, Les Ulis, 2002, Mathématiques.

[Sab06]__ Hypergeometric periods for a tame polynomial, Port. Math. (N.S.) 63 (2006), no. 2, $173-226$.

[Sab08]__ Fourier-Laplace transform of a variation of polarized complex Hodge structure., J. Reine Angew. Math. 621 (2008), 123-158.

[Sab18a]_ Irregular Hodge theory (with the collaboration of Jeng-Daw Yu), Mém. Soc. Math. Fr. (N.S.) (2018), no. 156 , vi +126

[Sab18b] _ Some properties and applications of Brieskorn lattices, Journal of Singularities 18 (2018), $239-248$.

[Sai88] Morihiko Saito, Modules de Hodge polarisables, Publ. Res. Inst. Math. Sci. 24 (1988), no. 6, 849-995 (1989).

[Sai90] Mixed Hodge modules, Publ. Res. Inst. Math. Sci. 26 (1990), no. 2, 221-333.

[Sch73] Wilfried Schmid, Variation of Hodge structure: the singularities of the period mapping, Invent. Math. 22 (1973), 211-319.

[Sha18] Yota Shamoto, Hodge-Tate conditions for Landau-Ginzburg models, Publ. Res. Inst. Math. Sci. 54 (2018), no. 3, 469-515.

[Sim90] Carlos T. Simpson, Harmonic bundles on noncompact curves, J. Amer. Math. Soc. 3 (1990), no. 3, 713-770.

[SY15] Claude Sabbah and Jeng-Daw Yu, On the irregular Hodge filtration of exponentially twisted mixed Hodge modules, Forum Math. Sigma 3 (2015), e9, 71 pp.

[SY18]__ Irregular Hodge numbers of confluent hypergeometric differential equations, preprint arXiv:1812.00755 [math.AG], 2018.

[Yu14] Jeng-Daw Yu, Irregular Hodge filtration on twisted de Rham cohomology, Manuscripta Math. 144 (2014), no. 1-2, 99-133.

E-mail address: alberto.castano@usc.es

E-mail address: christian.sevenheck@mathematik.tu-chemnitz.de 
Instituto de Matemáticas, Universidade de Santiago de Compostela, 15782 Santiago de Compostela (Spain); Fakultät für Mathematik, Technische Universität Chemnitz, 09107 Chemnitz (Germany)

Fakultät für Mathematik, Technische Universität Chemnitz. 09107 Chemnitz (Germany) 